\title{
Characterization and Modeling of Atrioventricular Conduction during Atrial Fibrillation
}

\author{
Batiste Andreu Martínez Climent
}

Supervisors:

Dr. María de la Salud Guillem Sánchez

Dr. José Millet Roig

May, 2011 



\section{Agradecimientos}

La consecución de la presente tesis hubiese sido completamente imposible sin la colaboración de numerosas personas que me han ayudado, enseñado y apoyado durante estos años. Quiero empezar agradeciendo el apoyo recibido por todos los integrantes del grupo de Bioingeniería del instituto ITACA de la Universidad Politécnica de Valencia desde que empecé a colaborar con ellos en 2005. De todos los que durante este tiempo han pasado por el laboratorio he aprendido muchísimo y tengo un más que grato recuerdo: Jose Luis Espí, José Laparra, Jose Antonio Belloch, Laura Maestro, Vicky Donis, Cinta Barber, Amparo Pomar, María Mozo, Lola Guerrero, Lydia Dux, Martina Srutova, Pietro Bonizzi, Lorena Rausell, Jesús Vila y Xavier Ibáñez. Y por supuesto quiero mandar un caluroso agradecimiento a todos con los que sigo trabajando día a día y de los que cada día aprendo nuevas cosas: Jorge Pedrón, Alejandro Liberos, Eduard Roses, Toni Guill, Alvaro Tormos, José Antonio Pérez, Toni Cebrián, Paco Castells, Conrado Calvo, Amador Ayala, María Vinaches, Cristina Rojas, Adolfo Fonseca, Jesús Escrivá y Miguel Rodrigo. Comentario a parte merece José Millet del cual he aprendido innombrables cosas y cuyo espíritu entusiasta y su capacidad de encajar los problemas con tranquilidad son virtudes que querría tener la capacidad de imitar y que me acompañasen durante el resto de mi carrera.

Además del apoyo de todos los compañeros del laboratorio, la realización de las diversas estancias de investigación ha sido imprescindible para que esta tesis haya llegado a buen término. Junto con el Dr. Andreas Bollmann entré en el mundo de la ciencia, de él aprendí algunas de las lecciones más valiosas entre las que quiero destacar el ímpetu por colaborar y aprender siempre con los mejores estuviesen en el laboratorio de al lado o a $5000 \mathrm{~km}$. Este afán por conseguir enriquecer nuestras investigaciones con el apoyo de algunos de los más reputados expertos del mundo me ha llevado a Milán junto con a la Dra. Valentina Corino y al Dr. Luca Mainardi, a Suecia con los Drs. Martin Stridth y Leif Srnmo, a Cleveland junto con los Drs. Youhua Zhang y Todor Mazgalev y a Michigan junto con los Drs. Omer Berenfeld y José Jalife. Cada una de esas estancias me ha enriquecido no sólo como investigador sino como persona. Poder colaborar con investigadores tan reputados a la vez que grandes personas ha sido un regalo que es difícil de describir en unas pocas palabras pero que viajará 
conmigo el resto de mi vida. Junto con las colaboraciones internacionales, en mi por ahora corta carrera como investigador he tenido la suerte de trabajar con grandes investigadores españoles entre los que quiero destacar a los Drs. Felipe Atienza, Aurelio Quesada y Javier Chorro por confiar en nosotros pese a nuestra juventud, ayudarnos con nuestras limitaciones y descubrirnos la parte más humana de la práctica clínica y la investigación.

En el futuro, ojalá tenga la suerte de poder seguir contando con la colaboración de todos estos magníficos científicos y espero que con muchos más. Pero de lo que estoy seguro que contaré es del apoyo de todos aquellos que siempre han estado a mi lado apoyándome pasase lo que pasase, dándome ánimo y luchando por mí. A mis padres les debo todo lo que soy. Desde el día que nací no han dejado de mostrarme el camino por el que construir una vida plena, no han dejado de esforzarse por conseguir que Amanda y yo seamos felices y para ello, además de trabajar muy duro para que nunca nos faltase nada, nos han regalado fantásticos momentos junto a ellos. Ojala algún día, mi siempre vibrante, extrovertida y querida hermana Amanda y yo, seamos capaces de llegar a devolverles aunque solo sea el $1 \%$ de todo lo que ellos nos han entregado. También quiero enviar el más cálido de mis agradecimientos a mis abuelos Salvador y Elvira cuyos esfuerzos en la vida me han enseñado a luchar por conseguir lo que uno desea, y a mi siempre idolatrado abuelo Andreu el cual me ha inculcado un espíritu crítico enseñándome a ver las cosas desde todos los puntos de vista, tratando de entender a los que no piensan como uno mismo y siendo crítico con aquellos que sí lo hacen. Un especial abrazo quiero enviar a mi abuela Carmen a la que siempre llevo en mi recuerdo más tierno.

El más fuerte de mis agradecimientos quiero enviárselo a la persona que me ha regalado los que por ahora han sido los mejores días de mi vida pero que seguro serán superados por los que están por llegar. Podría pasarme toda la eternidad buscando las palabras para describir lo importante que María es para mí y solo sería capaz de esbozar aquello que me hace sentir. Porque sin ti nada de esto hubiese tenido sentido, porque cada día te admiro con mayor devoción, porque siempre tienes la palabra justa, la idea genial, porque eres capaz de soportar mis no pocas manías y defectos, porque día a día consigues hacerme más y más feliz y porque sí, porque te quiero y quiero pasar el resto de mi vida aprendiendo día a día junto a ti. 


\section{Acronyms}

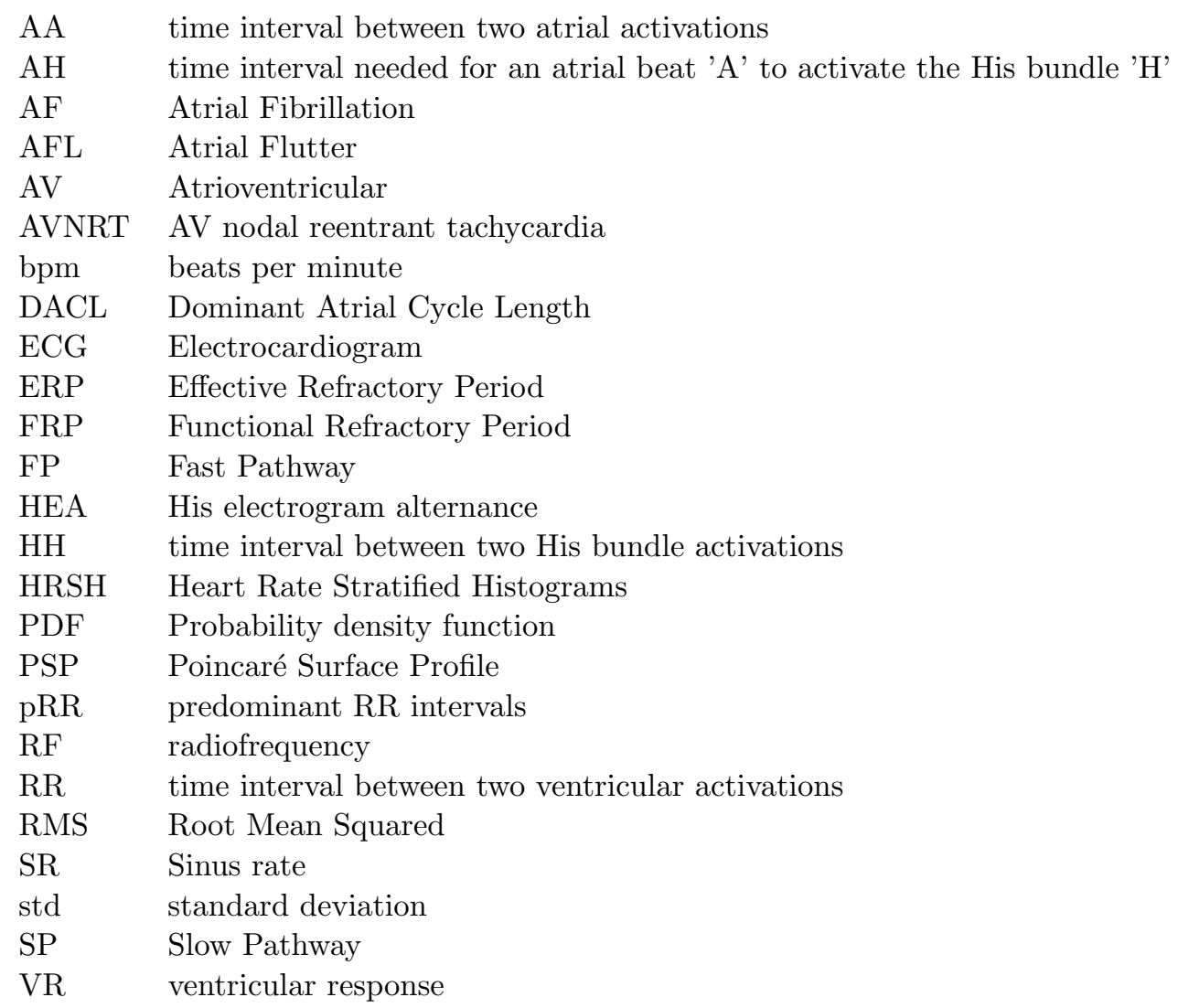





\section{Contents}

Agradecimientos $\quad$ i

Acronyms

Sumari $\quad$ xi

Sumario xiii

Abstract $\quad$ Xv

1 Introduction 1

1.1 Motivation ..................... 1

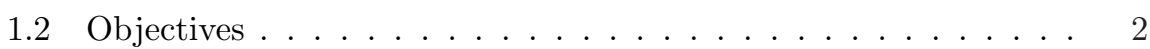

1.3 Structure of the thesis ................. . . 4

$2 \begin{array}{lll}2 & \text { State of the art } & \mathbf{7}\end{array}$

2.1 Introduction to Electrophysiology . . . . . . . . . . . . 8

2.1.1 The Heart . . . . . . . . . . . . . . . . 8

2.1.2 The Atrio-Ventricular Node . . . . . . . . . . . . . 10

2.1.3 The Dual AV Nodal Physiology . . . . . . . . . . . . . 12

2.1.4 Atrial Fibrillation . . . . . . . . . . . . . . . 13

2.1.5 Epidemiology . . . . . . . . . . . . . 15

2.1.6 Treatment of Atrial Fibrillation . . . . . . . . . . . 16

2.2 Analysis of the Ventricular Response during AF . . . . . . . . . . 19

2.3 Mathematical Modeling of AV conduction time . . . . . . . . . 22

2.3.1 Models of the AV time . . . . . . . . . . . . . 23

2.3.2 Models of the RR interval series . . . . . . . . . . . . . 25

2.3.3 Models of AV node cells and anatomical structures . . . . 25

3 Poincaré Surface Profiles $\quad 27$

3.1 Introduction . . . . . . . . . . . . . . . . . . . . . . . 28

3.2 State of the Art . . . . . . . . . . . . . . . . . 29

3.2.1 RR Interval Histograms . . . . . . . . . . . . . . . . 29 
3.2 .2 Poincaré Plots . . . . . . . . . . . . . . . . . . . . . . 29

3.2 .3 Histographic Poincaré plot . . . . . . . . . . . . . 30

3.2.4 Detection of Preferential AV Node Conduction . . . . . . 30

3.3 Methods . . . . . . . . . . . . . . . . . . . . 32

3.3.1 Poincaré Surface and Cluster Identification _. . . . . . . 32

3.3.2 Poincaré Surface Profile . . . . . . . . . . . . . . . . 33

3.3.3 Short Time Variations on the Ventricular Response . . . . 34

3.3.4 Statistical Analysis . . . . . . . . . . . . . . . . . . . 34

3.4 Database and Preprocessing . . . . . . . . . . . . . . . . 34

3.5 Results . . . . . . . . . . . . . . . . . . . . 35

3.5.1 Poincaré Surface and Cluster Identification . . . . . . . 35

3.5.2 Poincaré Surface Profile. Comparison with Histograms . . 35

3.5.3 Poincaré Surface Profile and Transition Clusters in

Poincaré Surface . . . . . . . . . . . . . . . 38

3.5.4 Illustration of Short Time VR Variations vs Variation of

Preferential Conduction . . . . . . . . . . . . . . 39

3.5.5 Illustration of Drug Effects . . . . . . . . . . . . . 40

3.6 Discussion and Conclusion . . . . . . . . . . . . . . . . . 42

4 Role of the Atrial Rate $\quad 47$

4.1 Introduction . . . . . . . . . . . . . . . 48

4.2 Materials and Methods . . . . . . . . . . . . . . . 48

4.2.1 Study Population and Holter ECG Acquisition . . . . . . 48

4.2 .2 Analysis of the Atrial Activity . . . . . . . . . . . . 49

4.2.3 Analysis of the Ventricular Response . . . . . . . . . . . 50

4.2.4 Comparison between Atrial Cycle Length and

Predominant RR Intervals . . . . . . . . . . . . . . . . 50

4.2.5 Statistical Analysis . . . . . . . . . . . . . . . . . . 51

4.3 Results . . . . . . . . . . . . . . . . . . 51

4.3.1 Atrial Cycle Length and Predominant RR Intervals . . . . 51

4.3.2 Autonomic Tone Effects . . . . . . . . . . . . . . . . 53

4.3.3 Rate Control Effects . . . . . . . . . . . . . . . . . . . 54

4.4 Discussion . . . . . . . . . . . . . . . . 55

4.4 .1 Main Findings . . . . . . . . . . . . . . 55

4.4.2 Ventricular Response during Atrial Fibrillation . . . . . . 56

4.4.3 Atrioventricular Conduction Mechanisms . . . . . . . . 57

4.4 .4 Limitations . . . . . . . . . . . . . . . . . . . . . 58

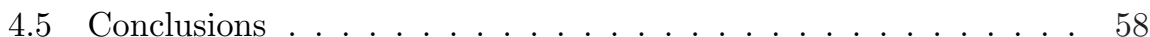

5 Generation of Realistic AA Interval Series 59

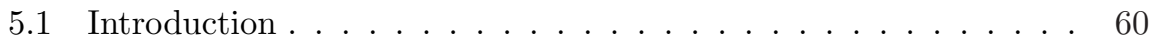

5.2 Methods . . . . . . . . . . . . . . . . . 61

5.2.1 Methodology for Generating Atrial to Atrial Interval Series 61

5.2 .2 Comparison with real AA interval series . . . . . . . . 63 
5.2.3 Role of Statistical Characteristics of the Atrial to Atrial Activity in the Ventricular Response during AF . . . . . . 64

5.3 Results . . . . . . . . . . . . . . . . . . . . 65

5.3.1 Accuracy of Pearson Type IV distribution . . . . . . . . 65

5.3.2 Accuracy of Generated AA interval series . . . . . . . . . 66

5.3.3 Role of Statistical Characteristics of the AA series in the Ventricular Response during AF . . . . . . . . . . 66

5.4 Discussion and Conclusion . . . . . . . . . . . . . . . . . . . 69

5.4 .1 Main Findings . . . . . . . . . . . . . . . . . . . 69

5.4.2 Comparison with other studies . . . . . . . . . . . 69

5.4 .3 Limitations . . . . . . . . . . . . . . . . . . . . 71

5.4 .4 Clinical Applications . . . . . . . . . . . . . . . 72

6 Model of Dual Pathway AV Node 73

6.1 Introduction . . . . . . . . . . . . . . . . . . . 74

6.2 Methods . . . . . . . . . . . . . . . . . 75

6.2.1 In-vitro Experiments . . . . . . . . . . . . . . . . 75

6.2.2 Experimental Pacing Protocols . . . . . . . . . . . . 75

6.2.3 Differentiation between Conduction Via "Slow" and "Fast" AV Node Pathways . . . . . . . . . . . . . . . 78

6.2.4 Mathematical Model of an AV Nodal Pathway . . . . . . 78

6.2.5 Incorporation in the Model of "Concealed" Conduction . 79

6.2.6 Description of the Dual Pathway Structure . . . . . . . 80

6.2.7 Calculation of Model's Constants . . . . . . . . . . . 80

6.2.8 Validation of the Model . . . . . . . . . . . . . . . . 83

6.3 Results . . . . . . . . . . . . . . . . . . . . . . 83

6.3.1 Conduction Curve Fitting and Model Equation Constants 83

6.3.2 Validation of the Model in Predicting Wenckebach Periodicity: Comparison with Rabbit AV Node . . . . . . 84

6.3.3 Validation of the Model in Predicting Atrial Fibrillation: Comparison with Rabbit AV Node . . . . . . . . . . . 86

6.3.4 Model Predictions after Modifications of Dual Pathway Electrophysiology . . . . . . . . . . . . . . . . 8 87

6.3.5 Selective AV Nodal Ablations and Wenckebach Periodicity 87

6.3.6 Selective AV Nodal Ablations and Atrial Fibrillation . . . 89

6.3.7 Multimodal HH interval Histograms: Role of the Dual AV

Nodal Physiology . . . . . . . . . . . . . . . . . . . . . . 91

6.4 Discussion and Conclusion . . . . . . . . . . . . . . . . . . . 94

6.4 .1 Major Findings . . . . . . . . . . . . . . . . . . . . 94

6.4.2 Comparison with Previous AV Node Models . . . . . . . . 95

6.4.3 Study Implications and Limitations _ . . . . . . . . . . 96 
7 Discussion and conclusion $\quad 99$

7.1 Discussion . . . . . . . . . . . . . . . . . . . . . 99

7.1.1 Limitations . . . . . . . . . . . . . . . 102

7.2 Conclusion . . . . . . . . . . . . . . . . . . 103

7.3 Guides for future work . . . . . . . . . . . . . . . . . . . . . 104

8 Contributions $\quad 107$

8.1 Publications ..................... 107

8.1.1 Main Contributions of This Thesis . . . . . . . . . . 107

8.1.2 Contributions Related to This Thesis . . . . . . . . . . 108

8.2 Framework of the Dissertation . . . . . . . . . . . . . . 109

8.2.1 Research Projects . . . . . . . . . . . . . . 110

8.2.2 International Research Stays . . . . . . . . . . . . 111

8.2 .3 Collaborations ................ 112 


\section{List of Figures}

2.1 Anatomy of the heart, valves and vessels . . . . . . . . . . 8

2.2 Intrinsic conduction system of the heart . . . . . . . . . . . 9

2.3 Anatomy of the triangle of Koch . . . . . . . . . . . . . . . . 11

2.4 ECG tracings during sinus rhythm and atrial fibrillation . . . . . 14

2.5 Conceptual models of atrial fibrillation . . . . . . . . . . . . . . . 14

2.6 Example of Inferior His Electrogram . . . . . . . . . . . . . . 18

2.7 Poincaré plots . . . . . . . . . . . . . . . . . . . 20

2.8 Clusters in the Poincaré plots . . . . . . . . . . . . . . . . 21

2.9 Wenckebach Periodicity . . . . . . . . . . . . . . . 23

2.10 Family of AV node conduction curves . . . . . . . . . . . . 24

3.1 Examples of Poincaré plot with RR intervals obtained during 24

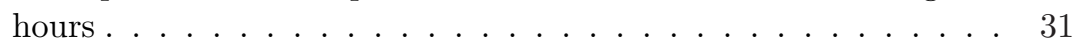

3.2 24-hour Poincaré Surface and RR interval histogram . . . . . . . 36

3.3 Difference between the positions of each peak detected by means of RR histograms or PSP . . . . . . . . . . . . . . . . 38

3.4 Short-time variations of the Poincaré Surface Profile . . . . . . . 41

3.5 Illustration of drug effects . . . . . . . . . . . . . . . . . . 42

4.1 Examples of Poincaré Surface Profiles (PSP) . . . . . . . . . . 52

4.2 Examples of rate control treatment analyzed by Poincaré Surface Profile (PSP) . . . . . . . . . . . . . . . . . 55

5.1 Schematic representation of the procedure used to generate realistic AA . . . . . . . . . . . . . . 6 . . . . . 63

5.2 Accuracy of Pearson type IV distribution . . . . . . . . . . . . 65

5.3 Example of AA interval series generation . . . . . . . . . . . . 67

5.4 Statistical parameters of generated AA series. . . . . . . . . 68

5.5 RR interval histograms illustrating the role of each statistical parameter of the AA interval series . . . . . . . . . . 70

6.1 Schematic presentation of atrial and ventricular activation periods 77

6.2 Illustration of different conduction interaction scenarios . . . . . 81 
6.3 Family of conduction curves for the FP and SP . . . . . . . . . 82

6.4 Model predictions . . . . . . . . . . . . . . . . 85

6.5 Wenckebach periodicity . . . . . . . . . . . . 88

6.6 An example of 6:5 Wenckebach periodicity . . . . . . . . . . . 89

6.7 Systematic analysis of the atrial AA pacing intervals producing Wenckebach periodicity ............... 90

$6.8 \mathrm{FP}$ and $\mathrm{SP}$ ablation . . . . . . . . . . . . . . . . . . 91

6.9 Atrial rate and $\mathrm{HH}$ interval histograms . . . . . . . . . . . . . 93 


\section{Sumari}

La fibrilació auricular (FA) és una de les arítmies cardíaques més comuns, que afecta al voltant del $10 \%$ de la població de més de 70 anys. En FA, els impulsos elèctrics auriculars generats per el node sinusal són substituts per impulsos elèctrics desorganitzats. Això està associat amb un bombardeig irregular de activacions auriculars cap al node AV. Atès que el node AV no pot conduir totes aquestes activacions, algunes d'elles són bloquejades en el node. Aquesta propietat de filtratge que té el node és fonamental per mantenir el ritme cardíac en un rang compatible amb la vida. No obstant això, la resposta ventricular durant FA presenta intervals RR (temps entre dues activacions) més curts i irregulars que durant ritme sinusal. Aquest ràpid i variable ritme cardíac pot causar alguns dels símptomes més severs d'aquesta arítmia. Com que el node AV es l'única estructura responsable per a la conducció dels batecs auriculars cap als ventricles, les estratègies terapèutiques per controlar el ritme cardíac durant FA tracten d'utilitzar i ajustar les propietats de conducció del node. No obstant això, segueix sense estar suficientment entès el paper que aquestes propietats de conducció juguen per controlar i modular la resposta ventricular durant FA.

Durant el desenvolupament d'aquesta tesi s'han investigat en diferents espècies $\mathrm{i}$ amb diverses tècniques algunes de les principals característiques de la conducció del node AV amb la intenció d'aportar major coneixement sobre aquesta intrigant estructura del cor. Específicament, s'ha analitzat un dels fenòmens més enigmàtics de la resposta ventricular durant FA: l'aparició de patrons de resposta ventricular multimodals en construir histogrames de RR obtinguts a partir de registres de llarga durada. En la literatura s'han suggerit diverses teories que poguessin explicar l'aparició d'aquests múltiples intervals RR predominants, en concret s'ha plantejat la possibilitat que representin múltiples del període refractari del node $\mathrm{AV}$ o que siguin deguts a l'existència d'una doble psicologia del node AV. En el desenvolupament d'aquesta dissertació es mostraràn alguns resultats incompatibles amb aquestes teories, raó per la qual es presenta i defensa una nova hipòtesi que suggereix que els intervals RR predominants estan relacionats amb el procés fibrilatori auricular. Per a validar aquesta nova hipòtesi s'ha treballat en dues vessants, una per confirmar empíricament les nostres suposicions sobre registres clínics de llarga 
durada en humans i una altra per validar aquestes suposicions mitjanant el desenvolupament i utilització d'un model matemàtic funcional del procés fibrilatori auricular i de la conducció auriculo-ventricular.

A la primera part de la tesi, es van analitzar senyals d'ECG registrades de 24 hores preses en pacients amb FA. Es va detectar el nombre i la posició dels intervals RR predominants, aquestes característiques es van comparar amb la mitjana i la desviació estàndard de la longitud de cicle dominant en la auricular. Això ens va permetre demostrar una relació estadística entre la longitud dels intervals RR predominants i els múltiples de la longitud de cicle auricular.

A la segona part de la tesi es van utilitzar registres epicàrdics, presos en humans i en conills, per modelar matemàticament el procés fibrilatori auricular i la conducció auriculoventricular. Mitjanant la utilització d'aquests models es va poder demostrar el paper fonamental que juga la longitud del cicle d'activació auricular. S'ha demostrat com durant FA el node AV s'encarrega principalment conduir activacions a un ritme múltiple del el ritme auricular mitjà. També s'ha comprovat la importància que té l'organització del bombardeig auricular a l'hora d'obtenir un o altre patró d'activació ventricular. A més, els models matemàtics desenvolupats han estat útils per clarificar algunes peculiaritats complexes de la conducció a través del node AV. El model s'ha utilitzat per a explicar com la interacció entre el camí ràpid i lent del node AV pot ser responsable de la generació del ritmes de Wenckebach atípics (ritmes ventricular irregulars durant activacions auriculars regulars). Per últim, el model presentat permet una fàcil ablació del camí ràpid o lent de conducció en el mateix cor per tant ha permès avaluar els efectes clínics de l'ablació la qual s'han presentat a la literatura com una possible eina terapèutica per controlar el ritme ventricular durant FA. 


\section{Sumario}

La fibrilación auricular (FA) es una de las arritmias cardiacas más comunes, la cual afecta alrededor del $10 \%$ de la población de más de 70 años. En FA, los impulsos eléctricos auriculares generados por el nodo sinusal son sustituidos por impulsos eléctricos desorganizados. Esto está asociado con un bombardeo irregular de activaciones auriculares hacía el nodo AV. Dado que el nodo AV no puede conducir todas estás activaciones, algunas de ellas son bloqueadas en el nodo. Esta propiedad de "filtrado" que tiene el nodo es fundamental para mantener el ritmo cardiaco en un rango compatible con la vida. Sin embargo, la respuesta ventricular durante FA presenta intervalos RR (tiempo entre dos activaciones) más cortos e irregulares que durante ritmo sinusal. Este rápido y variable ritmo cardiaco puede causar algunos de los síntomas más severos de esta arritmia. Al ser el nodo AV la única estructura responsable para la conducción de los latidos auriculares hacia los ventrículos, las estrategias terapéuticas para controlar el ritmo cardiaco durante FA tratan de utilizar y ajustar las propiedades de conducción del nodo. Sin embargo, sigue sin estar suficientemente entendido el papel que dichas propiedades de conducción juegan para controlar y modular la respuesta ventricular durante FA .

Durante el desarrollo de la presente tesis se han investigado en diferentes especies y con diversas técnicas algunas de las principales características de la conducción del nodo AV con la intención de aportar mayor conocimiento sobre esta intrigante estructura del corazón. Específicamente, se ha analizado uno de los fenómenos más enigmáticos de la respuesta ventricular durante FA: la aparición de patrones de respuesta ventricular multimodales al construir histogramas de RR obtenidos a partir de registros de larga duración. En la literatura se han sugerido diversas teorías que pudiesen explicar la aparición de estos múltiples intervalos RR predominantes. En concreto se ha planteado la posibilidad de que representen múltiplos del periodo refractario del nodo AV o que sean debidos a la existencia de una doble fisiología del nodo AV. En el desarrollo de la presente disertación se mostrarán algunos resultados incompatibles con dichas teorías, razón por la cual se presenta y defiende una nueva hipótesis que sugiere que los intervalos $R R$ predominantes están relacionados con el proceso fibrilatorio auricular. Para poder validar está nueva hipótesis se ha trabajado en dos vertientes, una para confirmar 
empíricamente nuestras suposiciones sobre registros clínicos de larga duración en humanos y otra para validar dichas suposiciones mediante el desarrollo y utilización de un modelo matemático del proceso fibrilatorio auricular y de la conducción auriculo-ventricular.

En la primera parte de la tesis, se analizaron registros de ECG de 24 horas tomados en pacientes con FA. Se detectó el número y la posición de los intervalos RR predominantes, estas características se compararon con la media y la desviación estándar de la longitud de ciclo dominante en la auricula. Este hallazgo nos permitió demostrar una relación estadística entre la longitud de los intervalos $\mathrm{RR}$ predominantes y los múltiplos de la longitud de ciclo auricular.

En la segunda parte de la tesis se utilizaron registros epicárdicos, tomados en humanos y en conejos, para modelar matemáticamente el proceso fibrilatorio auricular y la conducción auriculoventricular. Mediante la utilización de estos modelos se pudo demostrar el papel fundamental que juega la longitud del ciclo de activación auricular. Se ha demostrado como durante FA el nodo AV se encarga principalmente conducir activaciones a un ritmo múltiplo del el ritmo auricular medio. También se ha comprobado la importancia que tiene la organización del bombardeo auricular a la hora de obtener uno u otro patrón de activación ventricular. Además, los modelos matemáticos desarrollados han sido útiles para clarificar algunas peculiaridades complejas de la conducción a través del nodo AV. El modelo se ha utilizado para explicar como la interacción entre el camino rápido y lento del nodo AV puede ser responsable de la generación de ritmos de Wenckebach atípicos (ritmos ventriculares irregulares durante activaciones auriculares regulares). Por último, el modelo presentado ha sido utilizado para emular el efecto de la ablación del camino rápido o lento de conducción en el mismo corazón, permitiendo evaluar los efectos clínicos de la ablación, los cuales se han presentado en la literatura como una posible herramienta terapéutica para controlar el ritmo ventricular durante FA. 


\section{Abstract}

Atrial fibrillation (AF) is one of the most common cardiac arrhythmias, affecting about $10 \%$ of the population older than 70 years. In AF, the normal electrical impulses that are generated by the sinoatrial node are overwhelmed by disorganized electrical impulses within the atria. This is associated with an irregular bombarding of the atrial activity into the AV node. Since the AV node is not able to conduct all atrial activations, some of them are blocked within the node. This "filtering" property of the AV node is fundamental to maintain the heart beating in a range compatible with the life. However, the ventricular response during $\mathrm{AF}$ presents shorter and more irregular $\mathrm{RR}$ intervals (time between two heart beats) than during normal sinus rhythm. This fast and irregular ventricular rate can cause some of the most severe symptoms of this arrhythmia. Since the AV node is the only normal structure responsible for the conduction of atrial impulses to the ventricles, the strategy of control the heart rate during AF essentially deals with efforts to utilize and adjust conduction properties of the node. However, the role of AV nodal conduction properties in controlling and modulating the ventricular response during AF is not well understood.

During the development of the present thesis different characteristics of the $\mathrm{AV}$ conduction have been investigated in different species and with different techniques with the aim of throwing light upon this intriguing structure of the heart. Specifically, we analyzed one of the most intriguing behaviors of the ventricular response patterns during AF; when constructing RR-interval histograms obtained from Holter recordings with persistent AF, uni-, bi-, or multimodal RR distribution patterns can be found. These predominant RR intervals have been suggested in the literature to be multiples of the refractory period of the AV node or caused by the existence of a dual AV node physiology. However, during the development of this thesis some results incompatible with these theories have been presented, therefore we present and defend a novel hypothesis suggesting that predominant RR intervals are related to the atrial fibrillatory process. In order to validate this hypothesis we have used two main approaches, one to empirically confirm our assumptions by using real human clinical recordings and another to validate those assumptions by using mathematical models of the atrial fibrillatory process 
and the atrioventricular conduction.

In the first part of the present dissertation, Holter ECG signals from patients with AF were analyzed. Number and position of predominant RR intervals were detected and compared with mean and standard deviation of the dominant atrial cycle length. It allowed us to demonstrate a statistical relation between the position of predominant $\mathrm{RR}$ intervals of the multiples of the dominant atrial cycle length.

In the second part of this thesis, epicardial recordings from humans and rabbits were used to mathematically model the atrial fibrillatory process and the atrioventricular conduction. By using these mathematical models the fundamental role of the mean atrial to atrial interval activation has been illustrated; the $\mathrm{AV}$ conduction during $\mathrm{AF}$ has been demonstrated to be confined to scaling the mean atrial rate. The importance of the organization of the atrial bombarding into the AV node has been studied. In added, developed mathematical models were also useful to elucidate some complex peculiarities of the conduction through the AV node during arrhythmias. The model has been applied to explain how the interaction between fast and slow pathways of the AV node may be responsible for the development of atypical Wenckebach rhythms (irregular periodic ventricular cycles during regular atrial activation). In addition, the presented model has allowed an easy modification of either fast and slow pathways in the same heart thus permitting evaluation of the effects of clinical ablations used as therapeutic tools for control of ventricular rate during $\mathrm{AF}$. 


\section{Chapter 1}

\section{Introduction}

1.1 Motivation

1.3 Structure of the thesis

\section{$1.2 \quad$ Objectives}

\subsection{Motivation}

Technological advantages in the field of electronics and computing are allowing the discovering of new methods and phenomena in all scientific areas. Medicine is one of the areas that has greatly benefited from the introduction of new recording techniques and mathematical computing which have resulted in a major increase in the knowledge of the biological systems during the last years. Cardiology is, among other disciplines in medicine, one of the fields in which both the diagnosis and treatment have been greatly improved during the last decades thanks to the advances in technical tools for the research.

Since 1906, when Tawara produced his monograph detailing the structure of the specialized muscular system responsible for atrioventicular conduction system $[1,2]$, for his doctoral dissertation, the mysteries of this most complex of all parts of the conduction system of the heart remain. The atrioventricular (AV) node is known as the "soul" of the heart, and whoever understands its anatomy and electrophysiology will unlock the key to understanding the anatomic and electrical fucntion of the heart itself.

During the development of the present dissertation we will focus on the analysis of the AV conduction during fast and irregular atrial activity such as atrial fibrillation $(\mathrm{AF})$. In $\mathrm{AF}$, the normal electrical impulses that are generated by the sinoatrial node are overwhelmed by disorganized electrical impulses within the atria. This is associated with an irregular bombarding of 
the atrial activity into the AV node. Since the AV node is not able to conduct all atrial activations, some of them are blocked within the node. This "filtering" property of the AV node is fundamental to maintain the heart beating in a range compatible with life. However, the ventricular response during $\mathrm{AF}$ presents a shorter and more irregular ventricular rhythm (e.g. RR intervals which is the time between two heart beats) than during normal sinus rhythm [3]. This fast and irregular ventricular rate associated with AF can cause some of the most severe symptoms such as palpitation, chest discomfort, light-headiness and syncope. In fact, the rapid and irregular heart rate may result in the heart being unable to provide adequate blood flow and oxygen delivery to the rest of the body with all the associated long-term problems. And even worse, long-term tachycardia resulting from the uncontrolled ventricular rate may lead to an induced cardiomyopathy [3].

Current AF management guidelines suggest that there are fundamentally two ways to manage this rhythm disorder: to restore and maintain sinus rhythm or to allow AF to continue and ensure that ventricular rate is controlled [4]. Given similar outcomes between rhythm and rate control strategies, rate control seems an attractive endpoint for the treatment of $\mathrm{AF}$ in a large number of patients [5]. Since the AV node is the only normal structure responsible for the conduction of atrial impulses to the ventricles, the strategy of rate control during AF essentially deals with efforts to utilize and adjust the filtering properties of the node. However, the role of AV nodal conduction properties in controlling and modulating the ventricular response during $\mathrm{AF}$ is not completely understood. Which are the mechanisms that produces different populations of preferential $\mathrm{RR}$ intervals? Which is the role of the atrial fibrillatory process in the ventricular response? or How the dual AV nodal pathway physiology affects the ventricular response during AF? These are some of the questions that were in the basis of the motivation of the present thesis. During the development of the present thesis different characteristics of the AV conduction have been investigated in different species and with different techniques with the aim of throwing light upon this intriguing structure of the heart.

\subsection{Objectives}

The goal of the present thesis is to help in the understanding of some of the intriguing AV node mechanisms, since a deeper knowledge of the atrioventricular behavior may be crucial for an appropriate treatment selection. Specifically, we analyzed one of the most intriguing behaviors of the ventricular response patterns during AF; when constructing RR-interval histograms obtained from Holter recordings with persistent AF, uni-, bi-, or multimodal RR distribution patterns can be found. These predominant RR intervals have been suggested in the literature to be multiples of the refractory period of the AV node or caused by the existence of a dual AV node physiology. However, during the development of 
this thesis some results incompatible with these theories have been presented, therefore we present and defend a novel hypothesis suggesting that predominant RR intervals are related to the atrial fibrillatory process. In order to validate this hypothesis we have used two main approaches, one to confirm empirically our assumptions by using real human clinical recordings and another to validate those assumptions by using mathematical models of the atrial fibrillatory process and the atrioventricular conduction.

The aim of this dissertation is to evaluate the role of the atrial fibrillatory process and the atrioventricular conduction mechanisms in the ventricular response during AF. In order to achieve this main objective different sub-objectives were established:

- Development of a methodology for the characterization of the ventricular response during atrial fibrillation.

In order to achieve this objective, 24-hour Holter ECG recordings will be obtained. These recordings will be used to analyze the ventricular response pattern during AF. Novel non-linear signal processing tool will be presented and evaluated to characterize the ventricular response pattern of each specific patient and its time evolution.

- Evaluation of the role of the atrial fibrillatory activity in the ventricular response during $\mathrm{AF}$.

With this aim, two approaches will be used: 1) In the first, 24-hour Holter ECG will be analyzed by means of the new methodology developed for the identification of the ventricular response pattern. Most probable ventricular activation intervals will be compared with the mean atrial rate extracted from the surface ECG. A possible statistical relationship between the mean atrial rate and the duration of predominant RR intervals will be tested. 2) In the second approach, intracardiac recordings from the right and left atrium during AF will be used to present a methodology to generate synthetic Atrial to Atrial interval series with realistic statistical moments. Different synthetic AA interval series generated with this methodology will be applied in a functional model of the atrioventricular node to evaluate the role of the atrial fibrillatory activity.

- Evaluation of the role of the dual AV nodal physiology in the ventricular response during $\mathrm{AF}$.

In order to accomplish this objective, we will present a novel mathematical model of atrioventricular conduction that incorporates dual AV nodal pathway physiology. Experiments performed on 5 rabbit atrial-AV nodal preparations will be used to develop and test the presented model. The ability to predict AV conduction time and the interaction between FP and SP wavefronts will be analyzed during 
regular and irregular atrial rhythms (e.g., atrial fibrillation). This model will allow us to evaluate the role that each AV node pathway plays in the ventricular response pattern during AF. The presented model will be also used to explain some of the unclear mechanisms of the AV node as the typical and atypical Wenckebach periodicity or the concealed conduction.

\subsection{Structure of the thesis}

This thesis is structured in the following chapters:

Chapter 2: State of the art: This is a review chapter that reflects the state of the art in the different disciplines that are involved in this work. Current knowledge of the atrioventricular conduction and mechanisms underlaying AF are summarized in this chapter. In added, signal processing methods employed in the literature for the characterization of the ventricular response during AF are summarized. Finally, a short description of the different mathematical models of the atrioventricular conduction presented in the literature is included.

Chapter 3: Poincaré Surface Profiles of RR intervals: In this chapter we present a novel methodology for the characterization of the ventricular response pattern during AF. Specifically, Poincaré Surface Profiles are described and compared with the classical histogram for the identification of more than one preferential RR interval population during AF.

Chapter 4: Role of the Atrial Rate: In this chapter the role of the atrial activation rate plays in the ventricular response during $\mathrm{AF}$ is evaluated using human surface recordings. It is illustrated how the position of predominant $\mathrm{RR}$ intervals has been shown to be correlated with multiples of the dominant atrial cycle length.

Chapter 5: Realistic Generation of Atrial to Atrial Interval: In this chapter, a novel methodology for the generation of realistic AA interval series during AF is proposed. The generated synthetic AA series are compared with real AA interval series recorded during electrophysiological studies. In added, the role of the AA interval series characteristics in the ventricular response is evaluated.

Chapter 6: Functional Mathematical Model of Dual Pathway AV nodal Conductions: In this chapter, a novel functional model of the AV node that includes the dual pathway AV node physiology based on experimental data is presented. The inclusion of fast pathway (FP) and slow pathway (SP) conduction properties not only provided an excellent fit to the experimental database, but also helped to elucidate complex and 
still poorly understood peculiarities of conduction through the AV node during arrhythmias.

Chapter 7: Discussion and conclusions: The contributions of this work are discussed in detail in this chapter, emphasizing the most relevant advances, but also their limitations. The fulfilment degree of the objectives established in the previous section is also analyzed in this chapter. Finally, some guides for future work are provided to overcome the limitations of this thesis and to extract the maximum profit from it.

Chapter 8: Contributions: The main scientific contributions derived from the work developed in this dissertation are listed in this section. The scientific framework in which this work has been involved is also described, including related research projects and international collaborations. 



\section{Chapter 2}

\section{State of the art}

\subsection{Introduction to Electrocardiography}

\subsection{Ventricular Response during Atrial Fibrillation}

\subsection{Mathematical Modeling of Atrio-ventricular Conduction}

In this chapter the main areas that are covered in this dissertation are explained, providing a sufficient background of the main concepts that are involved in the following chapters.

Fundamental concepts about heart electrophysiology and specifically the atrioventricular conduction are described. In addition, current knowledge of the pathophysiology of atrial fibrillation is summarized in this chapter together with some unresolved issues which are tightly related with the content of this thesis.

The most relevant techniques that have been proposed in the literature for the characterization of the ventricular response during atrial fibrillation are described. These techniques include the analysis of RR interval series by means of non-linear signal processing methods such as the histogram, the heart-rate stratified histogram and the Poincaré plot.

Finally, a brief review of all the mathematical models that have been presented in the literature to reproduce the mechanisms and behaviors of the AV node has been included in the present chapter. 


\subsection{Introduction to Electrophysiology}

\subsubsection{The Heart}

The heart is a muscular organ that lies in the center of the thoracic cavity whose primary function is to pump blood throughout the body by repeated, rhythmic contractions.

The heart is composed mostly of cardiac muscle called myocardium. It is divided by a central wall, or septum, into left and right halves. Each half consists of an atrium, which receives blood returning to the heart from the blood vessels, and a ventricle which pumps blood out into the blood vessels. The right side of the heart receives blood from the tissues and sends it to the lungs for oxygenation. The left side of the heart receives newly oxygenated blood from the lungs and pumps it to tissues throughout the body. Although blood flow in the left and right side of the heart is separated, the two sides of the heart contract in a coordinated fashion: first the atria contract together, then the ventricles contract. The heart has four valves: between the right atrium and ventricle lies the tricuspid valve, and between the left atrium and ventricle is the mitral valve. The pulmonary valve lies between the right ventricle and the pulmonary artery, while the aortic valve lies in the outflow tract of the left ventricle. Anatomy of the heart, valves and vessels is depicted in Figure 2.1.

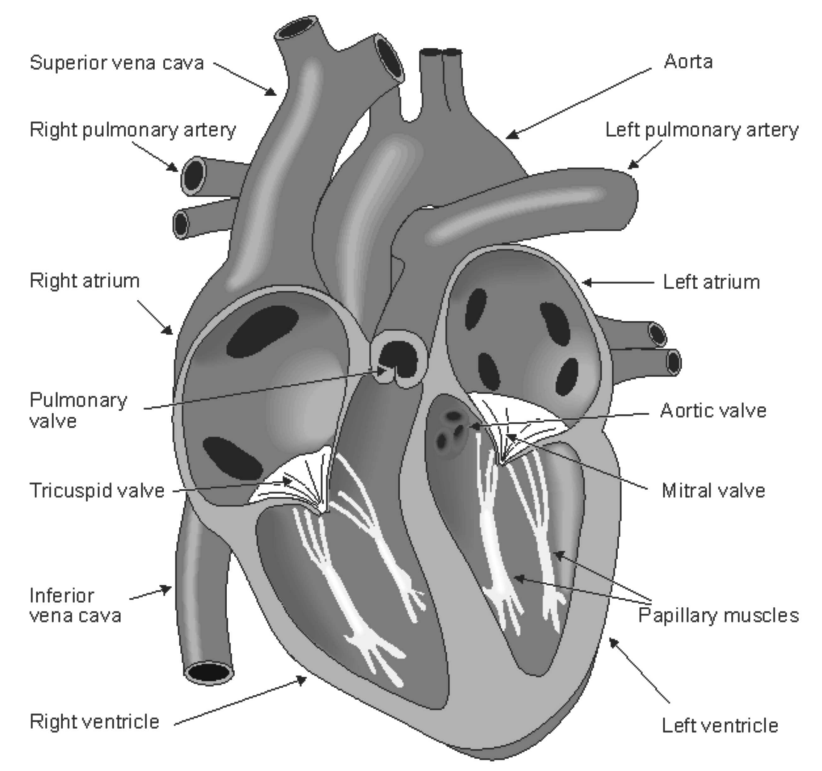

Figure 2.1: Anatomy of the heart, valves and vessels [6].

The rhythmic contractions of the heart depend on an electrical system which conducts electrical impulses along its conduction pathway. The marker 
of electrical stimulation, the action potential, is created by a sequence of ion fluxes through specific channels in the membrane of myocardial cells. If we were able to measure the intracellular potential with a voltmeter, the cathode measuring the potential inside the cell and the anode measuring outside, it could be observed that during a non-activity period the membrane voltage would remain constant around $-80 \mathrm{mV}$. In this state it is said that the cell is polarized, this is to say that the cell is at its resting potential. When a muscular cell is stimulated its membrane potential necessarily changes (there exists ion flow through the ionic channels of the membrane), characterized by a decrease in the normally negative resting voltage (depolarization) which triggers the activity of the cell, i.e. the contraction. After stimulation the membrane voltage returns to its original value (repolarization).

The impulse conducting system (Figure 2.2) consists of specialized cells that initiate the heart beat and electrically coordinate contractions of the heart chambers. The initiation of a cardiac cycle occurs in a small mass of specialized cells located in the wall of the right atrium with the ability to spontaneously depolarize and generate an action potential. This small mass of cells with pacemaker properties is called the sinoatrial (SA) node. Electrical impulses then spread rapidly through the internodal tracts that connect the SA node to the atrioventricular (AV) node, a group of autorhythmic cells near the floor of the right atrium. From the AV node, electrical impulses move into the bundle of His, which perforates the interventricular septum posteriorly. Within the septum, the bundle of His bifurcates into the left and right bundles. Finally, Purkinje fibers transmit the electrical impulses to the ventricular muscle. For a more detailed description of the anatomy and function of the heart see $[7,8]$.

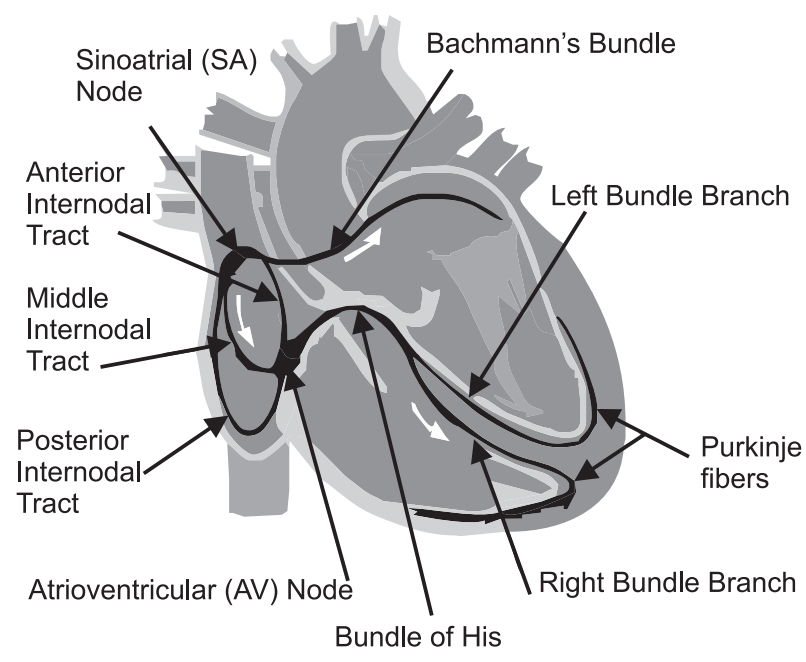

Figure 2.2: Intrinsic conduction system of the heart 


\subsubsection{The Atrio-Ventricular Node}

The atrioventricular node (AV) node is that part of the heart known to electrophysiologist as the specialized atrioventricular junction area. The inherent function of the AV node is to delay the cardiac impulse. Although it has been more than 100 years since Tawara [1] described the anatomic location and the morphologic structure of the AV node, the node has been the source of a remarkable number of controversies. Recently, several extensive reviews have been published trying to address what we know and do not know about the AV node $[9,10,11,12,13,14,15,16]$ (to mention just a few).

The AV describes a compact spindle-shaped network of cells arranged in a node connected to the His bundle, both being responsible for the only physiological atrioventricular axis of conduction. The AV node is found at the base of the atrial septum at the apex of a triangular area first illustrated by Koch [17]. This triangle is situated on the endocardial surface of the right atrium, is bordered anteriorly by the insertion of the septal leaflet of the tricuspid valve, and posteriorly by a fibrous tendon known as the tendon of Todaro (Fig. 2.3). This tendon is the fibrous subendocardial continuation of the Eustachian valve, and inserts into the atrial musculature separating the orifice of the coronary sinus from the fossa ovale. The apex of this triangle is formed superiorly by the junction of the anterior and posterior borders mentioned above, corresponding to the central fibrous body of the heart. The base of the triangle is formed by the orifice of the coronary sinus together with the vestibule of the right atrium supporting the septal leaflet of the tricuspid valve. This base is known to electrophysiologists as the septal isthmus [14].

Precise borders of the AV node cannot be determined. Thus, the general region of the triangle of Koch contains multilayered and very complex morphological structures that anatomically and functionally form a continuum between the atrium and the bundle of His. Although different authors usually use a different nomenclature, the AV junctional area is most frequently described as encompassing transitional cells, specialized nodal cells (AV node), lower nodal cells, and the penetrating AV bundle (bundle of His).

The term "transitional cells" is used broadly to describe the approaches from the working atrial myocardium to the AV node. It should be stressed that even though the term transitional might imply incorrectly that the region is outside the AV node, its functional importance for the impulse transmission should not be overestimated. The "typical" AV nodal cells comprise the socalled midnodal, or compact node region. These are the cells forming a dense discoid-shaped network for which Tawara coined the term "Knoted" (meaning "node"), frequently used for description of the entire region. In addition to being closely packed together ("compact"), the midnodal cells are typically the smallest, have random orientation [18] and contain few nexuses [19]. Lower nodal cells start property as this bundle of small cells that make scarce contacts with transitional cells. In the lower nodal bundle, the cells are longer and 


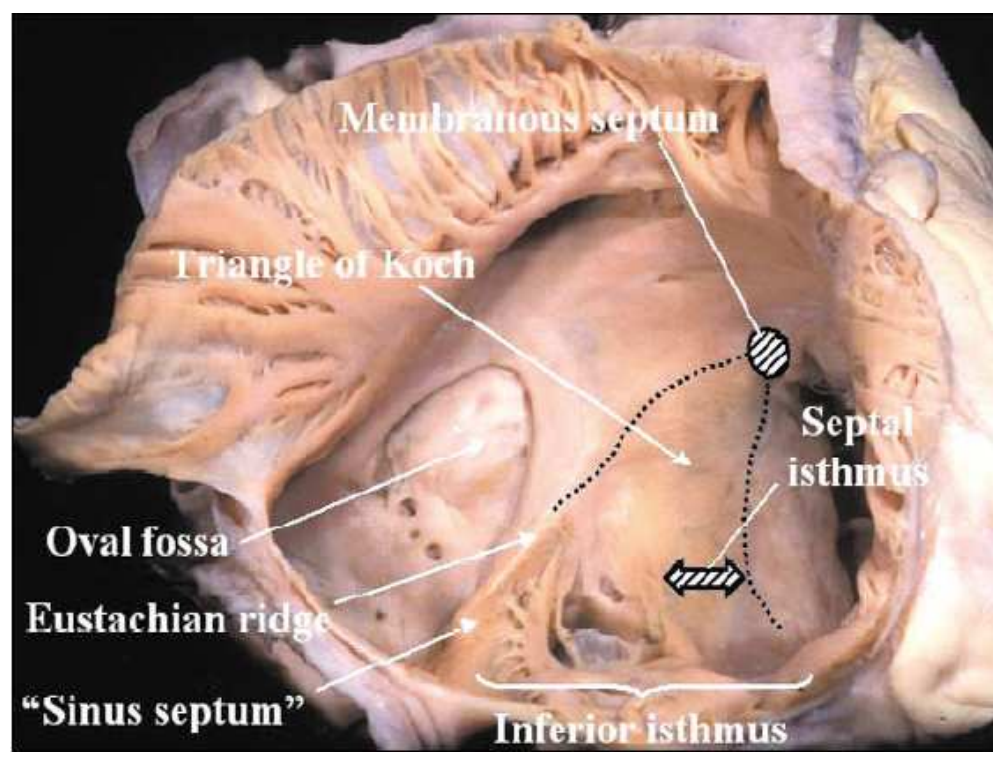

Figure 2.3: Anatomy of the triangle of Koch. The heart has been positioned in attitudinally appropriate orientation, and the right atrium opened through a window in the appendage. The septal surface of the atrium is located posteriorly, and the apex of the triangle of Koch (dotted lines) points upwards. The important atrial landmarks are shown. [11]. 
arranged more parallel to one another than in the compact node [15]. Closer to the compact node one can recognize larger individual bundles separated by fibrous tissue, and as the lower cells progress even more distally they form, without any sharp boundary, the penetrating AV bundle.

In the literature, based on the functionally and on the action potential morphology, several different AV node cell types have been defined including (A) atrial cells, (N) nodal cells and (H) Hiss cells, as well as intermediate cells types such as $\mathrm{AN}, \mathrm{NH}$, and other types [20, 21]. In general, the AN action potential morphology come from the cells in the transitional region and, as the name suggests, their action potentials are intermediate between the atrial cells and the nodal cells. The N cells, allegedly the "most typical" of the nodal cells, have slow rising and longer action potential. The NH cells are closer in appearance to the fast rising and long action potential of the His bundle [21]. Notice that there is no strict correspondence between these cellular types and the specific areas from which the action potential morphology are recorded, in fact, although the proportion of each cell types changes with the location, all of them seem to be present in all regions of the AV junction [22]. Additionally, some of these cells within the AV node can demonstrate triggered and automatic pacemaker activity.

\subsubsection{The Dual AV Nodal Physiology}

Moe et al. provided evidence for what they described as a dual-conduction atrioventricular conduction system in the hearts of dogs and rabbits [23]. This concept was soon applied to the human heart [24]. A simplified definition of the dual-AV node electrophysiology assumes that more than one wavefront exist at a given time in the triangle of Koch. Each one of these wavefronts may present different conduction properties (i.e. conduction velocity and refractory period). Gordon Moe et al. [23, 25] established that depending on the time from the last AV node activation (i.e. the coupling interval between 2 subsequent atrial impulses) the propagating wavefront may have different spatial and functional properties.

Let us assume that 2 functional pathways, $\alpha$ and $\beta$ are formed between the atrium and the bundle of His. They meet at some point and proceed after that as a final common pathway. During anterograde propagation (from atria to the ventricule) the cardiac impulse has the opportunity to proceed via any of the pathways. However, for the normal sinus beats (or beats with long preceding coupling intervals) the pathway $\beta$ provides a shorter, preferential route; thus a fast-pathway conduction takes place. The situation changes drastically when an impulse with short coupling interval occurs. For such a beat the $\beta$ pathway may be functionally blocked. In other words its refractory period is longer than the $A A$ interval. The atrial beat now proceeds via the $\alpha$-pathway all the way down to the bundle of His. What we have just explained is the classical manifestation of dual AV nodal electrophysiology. The way in which these pathways interact 
during fast and irregular atrial rates such as atrial flutter and atrial fibrillation remains not well understood. The efforts taken during the development of the present thesis to clarify this issue are summarized in chapter 6 .

Although the described functional nature of the dual AV nodal conduction systems appears to be self-evident, the anatomic substrate has remained much more elusive. The reader is referred to an excellent review which tries to addresses several misunderstoods on the concept of the duality of the nodal electrophysiology [16]. Anatomically the atrio-nodal transition has been described to encompass at least two characteristic approaches. [26]. One of them, the inferior approach, is formed as a continuation of the crista terminalis and the isthmus between the ostium of the coronary sinus and the septal leaflet of the tricuspid valve. The second, the superior approach, provides the connection with the interatrial septum. However, in view of the fact that these approaches have never been found to represent specialized and insulated conduction tracks [27], this morphological classification does not imply that two morphological pathways lead from the atrium into the AV node [28]. Therefore, the concept of the dual pathway AV nodal electrophysiology should be understood as a functional concept as described in electrophysiological studies.

\subsubsection{Atrial Fibrillation}

Atrial fibrillation (AF) is a supraventricular arrhythmia in which the electrical activation in the atria shows an uncoordinated pattern. This chaotic electrical activity results in a loss of effective contraction of the atria which is reflected on the surface ECG by a lost of the $\mathrm{P}$ wave that appears during sinus rhythm and the presence of AF waves (see Figure 2.4).

Normally, the heart rate is controlled by the SA node and adapts to body's needs in a range of 60 beats per minute at rest to 200 beats per minute at peak exercise. During AF, atrial cells fire at rates of 300 to 600 beats per minute. If each atrial impulse were conducted to the ventricles, such a rapid ventricular rate would result in a loss of the effective contraction of the ventricles and a rapid death. This is prevented by the filtering function of the AV node allowing only a part of the impulses to reach the ventricles. As a consequence of the irregular electrical activity of the atria and the effect of the AV node, the ventricular rate is also irregular (see Figure 2.4).

The mechanisms underlaying development and maintenance of AF are still not well understood. During the twentieth century different theories about the origin of AF coexisted: existence of a rapidly discharging atrial ectopic foci (Figure 2.5a), by a single reentry circuit (Figure 2.5b) or by multiple functional reentrant circuits (Figure 2.5c). For both the rapid focal and single reentrant circuit theories, irregularity is presumed to result from interactions between wavefronts produced by the main generator while for the multiple circuit reentry irregularity is a consequence of the primary arrhythmia mechanism [29]. 


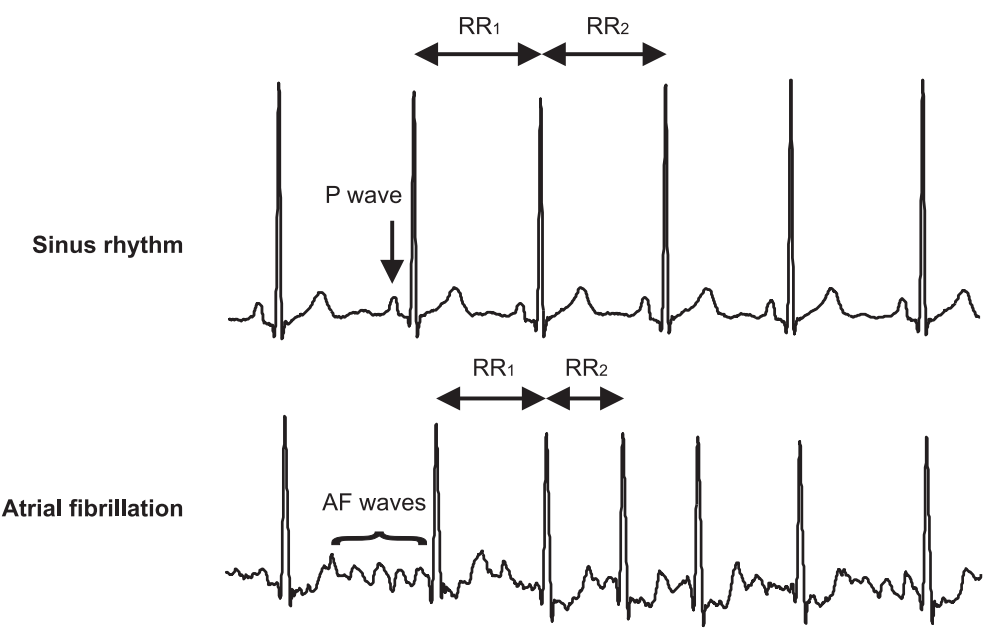

Figure 2.4: ECG tracings during sinus rhythm and atrial fibrillation
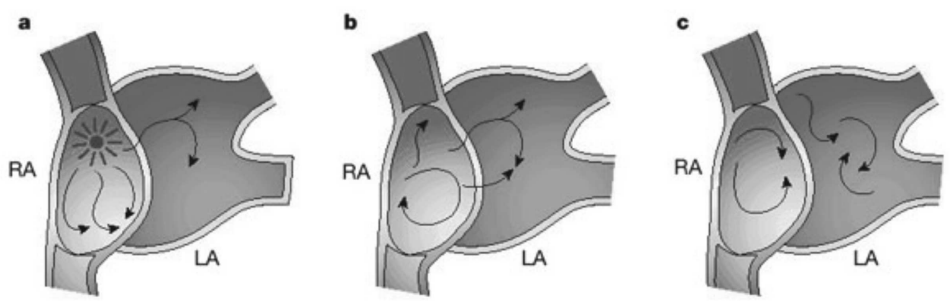

Figure 2.5: Conceptual models of atrial fibrillation. (a) ectopic foci, (b) single reentrant circuits and (c) multiple reentrant circuits [29] 
Recent observations on the initiation of AF from the area of the pulmonary veins [30] and optical mapping studies that found mother rotors and fibrillatory conduction as the mechanism underlying perpetuation of AF [31] suggest that a complex mechanism in which ectopic activity, single circuit reentry and wavefront fractionation may all be involved.

A better understanding of a number of processes collectively referred to as atrial remodeling has added to the complexity of the pathophysiology of AF [32]. Remodeling is the name given to the alteration of several properties in the atria as a consequence of AF that favors the easy of inducing and maintaining the arrhythmia [29]. Remodeling can be subdivided into electric, contractile and structural. Electrical remodeling refers to the alteration of electrophysiological properties in the atria that result in the shortening of atrial refractoriness. It develops within the first days of AF and contributes to an increase in stability of AF. Contractile remodeling refers to a loss of contractility in myocardial muscle that persists after the restoration of sinus rhythm. Atrial structural remodeling is associated to atrial fibrosis which occurs when AF is perpetuated during long periods of time. One of the main reasons to make distinction among types of remodeling is that the first two types of remodeling seem to be reversible, whereas the last is less so [33].

Clinicians distinguish three basic types of atrial fibrillation: paroxysmal, persistent and permanent [4]. Paroxysmal AF is characterized by AF episodes that initiate and terminate spontaneously. In this type of AF triggered activity appears to be the dominant factor and thus elimination of the trigger is expected to terminate the arrythmia. Persistent AF requires an intervention for termination to occur. In this type of AF, electrical remodeling is present while none or little structural remodeling exists and thus this form of AF is reversible. Permanent AF is characterized by extensive fibrosis in the atria that makes impossible to restore sinus rhythm despite aggressive therapy.

\subsubsection{Epidemiology}

Atrial fibrillation is the most common sustained arrhythmia, affecting an estimated 2.3 million adults in the United States [34]. The prevalence of atrial fibrillation increases markedly with age in older adults, from $<1$ per cent in adults younger than 55 to 10 per cent in adults older than 80 years. Projections are that by the year 2025, 3.3 million adults in the United States will have atrial fibrillation, with $>35$ per cent of affected persons 80 years or older.

In most patients, AF is associated with some other cardiac or non-cardiac process including hypertension, congestive heart failure, diabetes and previous myocardial infarction. However, some patients seem to have AF as their only diagnosis. In those patients genetic factors may be important [35], although genetic linkage has only been reported in a small part of the population with AF. 
Hospitalizations for atrial fibrillation as the principle diagnosis increased from 154.086 in 1985 to 376.487 in 1999 in the United States. In the same period, hospitalizations for atrial fibrillation as a secondary diagnosis increased from 787.750 to 2.283.673. In-hospital mortality among patients with a principle diagnosis of atrial fibrillation was $<1$ per cent in patients 35 to 74 years of age, 1.5 per cent in those 75 to 84 years of age and 3 per cent in patients 85 years or older [34].

\subsubsection{Treatment of Atrial Fibrillation}

Currently there are two broad strategic treatment options for AF: rhythm control and rate control [4]. For rhythm control, the treatment is directed toward restoring and maintaining sinus rhythm. The rhythm control strategy includes pharmacological treatments, as well as curative interventions such as the ablation of atrial regions responsible of the arrhythmia [30, 36] or a more radical surgical maze procedure [37]. The rhythm control will not be discussed in this thesis since it is not related with the main topic of the thesis: the atrioventricular conduction. Readers are invited to read some excellent reviewers where this topic is addressed [4].

For rate control, the intention is to slow ventricular rate while allowing AF to continue. Since the AV node is the only normal structure responsible for the conduction of atrial impulses to the ventricles, until now the strategy of rate control during AF essentially deals with efforts to utilize and adjust the filtering properties of the node [3]. This modification of the AV node conduction properties can be either non-destructive, or can use ablative techniques.

The basic electrophysiological properties of the atrioventricular transmission suggest that such modifications can include anatomic alterations of the atrionodal communications, medications that depress the impulse transmission within the AV node, as well as autonomic manipulations that produce negative dromotropic effect.

\section{Pharmacological modification of the AV node for rate control}

Drug therapy is the most common approach for rate control. A variety of drugs producing negative dromotropic effect through different mechanisms can be used for this purpose. Clinically best recognized are the calcium-channel antagonists, the $\beta$-blockers, and the digitalis drugs. Mechanistically all three group of drugs affect, through different ways, the slow calcium influx in the AV node cells. The choice of drugs should be based on the patient's clinical assessment, evaluation of underlying heart disease and drug contraindications $[4]$.

\section{AV node ablation and ventricular pacing}

AV node ablation is currently used as an alternative to control ventricular rate in patients in whom drug therapy is ineffective or drugs are not well tolerated due to side effects [38]. 
Since the AV node is the only normal route conducting atrial fibrillatory excitation to the ventricles, one radical solution is to ablate the AV junction and produce a permanent AV block. This requires the implantation of a ventricular pacemaker with all the associated problems and risks [3]

\section{Modification of AV node by partial ablation}

Early observations [39] in patients with Wolf-Parkinson-White (WPW) syndrome and AF revealed a bimodal pattern of RR histogram. One RR population was made up of the RR intervals with wide QRS representing ventricular activation through the accessory pathway. The other population of RR intervals corresponded to the intervals preceding narrow QRS complexes, i.e., beats conducted through the AV node. These observations were extended later to patients without accessory pathways, on the assumption that if dual pathways existed then RR intervals resulting from conduction via the FP and SP should have different distribution due to the different pathway's conduction properties [40].

By using the bimodal distribution existence as guidance, several studies have reported the effects of SP pathway ablation [41, 42, 43]. Despite the attractiveness of this phenomenon, the bimodal $R R$ interval distribution analysis does not provide full accuracy, and unimodal histogram pattern may still be associated with dual AV node pathways [41]. Therefore, while AV node modification should be expected in general to alter the distribution of the ventricular $R R$ intervals, it remains difficult to associate particular points in the histogram distribution with beats propagated via the FP or SP during AF.

Recently, a novel index of dual pathway electrophysiology has been introduced, termed "His electrogram alternans (HEA)". In rabbit heart experiments it was shown that this index could be used to determine on a beat-by-beat basis during $\mathrm{AF}$ the $\mathrm{AV}$ node conduction pattern (i.e. though FP or SP) $[44,45,46]$. Specifically, a low amplitude inferior bundle of His electrogram (IHE) was associated with FP conduction, while high-amplitude IHE indicates SP conduction. This index was validated during programmed electrical stimulation (e.g. generation of AV node conduction curve), as well as during atrial arrhythmia $[44,45,46]$.

In figure 2.6 an example of high-low IHE alternans recorded during experimental AF in rabbit heart preparation [44, 45, 46]. Successful SP modification prolonged the AV node ERP without changing the basic AV node conduction time, which is consistent with clinical SP ablation [3]. More importantly however, ablation in the SP domain completely eliminated the $\mathrm{SP}$-component of $\mathrm{AV}$ conduction during $\mathrm{AF}$, as objectively indicated by the removal of all high amplitude IHE (Fig. 2.6B). Predictably, this also resulted in a significant slowing of ventricular rate.

Since the HE alternans existed in all examined preparations, it provides strong evidence that dual pathway AV node electrophysiology is the normal feature of the AV conduction during AF. In particular, the index of HE alternans 

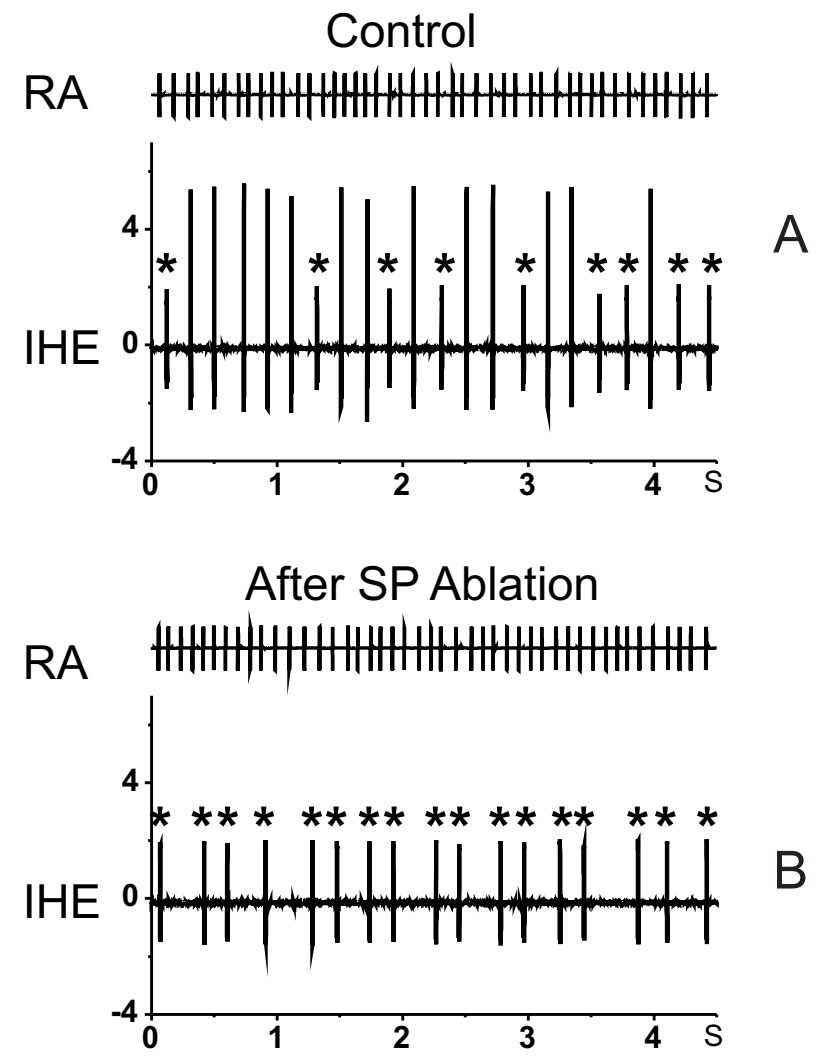

Figure 2.6: An example of high and low amplitude inferior His electrogram alternans recorded during experimental AF in a rabbit preparation before AV nodal modification. The His-spikes with low amplitudes (stars) indicate fast pathway conduction. (Panel B) After successful slow pathway modification, all high amplitude His spikes are eliminated, and the low amplitude IHE (stars) indicate exclusive fast pathway conduction during AF. Note that this also resulted in a significant slowing of ventricular rate [45]. 
detected SP and FP propagation even when the RR histograms exhibited only a single peak [47].

This novel index will be used in chapter 6 of the present thesis in order to model the atrioventricular conduction during AF incorporating for the first time the dual AV nodal physiology.

\subsection{Analysis of the Ventricular Response during Atrial Fibrillation}

During AF, the sinus node loses its pacemaker function, thus resulting in rapid and random atrial impulses. These fibrillatory impulses continuously bombard and penetrate the atrioventricular node to varying degrees, creating a notable variability on the AV nodal refractoriness [48] and in the ventricular response. This irregular ventricular response is often described as chaotic and without any form of patterning, nevertheless previous studies have shown that this process is not completely random [49]. Ventricular response during AF shows preferential conductions and different degrees of short time predictability [50]. Since the AV node is the structure responsible for the conduction of atrial impulses to the ventricle, the ventricular response during $\mathrm{AF}$ is mainly governed by the propagation properties of the node [3]. However, AV conduction mechanisms are not completely understood and, in particular, the role of the AV node physiology and the rate and irregularity of the atrial fibrillatory waves are still unknown. In the literature, characteristics of AV conduction have been investigated in different species and with different techniques [51]. In fact, signal processing tools are playing a relevant role for a deeper understanding of AF mechanisms and for quantifying AF patterns and properties $[49,51]$. In this section a short summarizing of non-linear signal processing methods used in the literature to evaluate the ventricular response are described. These methodologies will be applied during the development of the present thesis. A more in detail description of ventricular response signal processing tools including time-domain and spectral analysis methods could be found in [51].

Virtually in every patient with AF, standard 12-lead surface electrocardiograms (ECG) and/or Holter recordings are acquired, the main purposes being confirmation of arrhythmia presence and determination of ventricular rate. These long recordings are used for the evaluation of the AV node conduction characteristics and to adjust the rate control strategy in each specific patient [51,3].

AV conduction properties can be evaluated by constructing histograms obtained from Holter recordings of patients with paroxysmal or persistent AF. In fact, RR histograms during AF may evidence uni-, bi-, or multimodal RR distribution patterns $[3,49,40,43,42]$ which may indicate specific $\mathrm{AV}$ 
conduction mechanisms and may influence the most appropriate treatment [52]. In added, RR interval Histograms can be used to extract electrophysiological characteristics such as functional refractory period (FRP) noninvasively $[51,53]$.

As commented, RR histograms represent distribution of single intervals and are useful for a first evaluation of the ventricular response during AF but give no information about the sequence of intervals, i.e. the regularity of the ventricular rhythm. In a Poincaré plot, each $R R$ interval is plotted versus the preceding one $[54,55,56]$; it should be noted that this type of plot is also referred to as the Lorenz plot. It allows a beat-to-beat approach of HRV detecting pattern resulting from non linear processes and it was presented as a clinically useful tool for the study of AV nodal characteristics in AF. In fact, the pattern of such a plot can be inspected in order to distinguish AF form other supraventricular tachycardias such as atrial flutter where ventricular response is not as irregular as in AF. As shown in Fig. 2.7. During sinus rhythm (SR), successive RR intervals are centered around the main diagonal forming an ellipsoid-like pattern, and each $\mathrm{RR}$ interval is strongly dependent on the preceding RR interval. Atrial flutter is characterized by organized atrial dysfunction and occurs when an abnormal macroreentrant conduction circuit develops inside the atria, with an activation rate of $250-300 \mathrm{bpm}$. The AV node conducts a periodic number of these activations which become visible as clusters in the Poincaré plot; see Fig. 2.7b [52]. During AF, the irregularity of $\mathrm{RR}$ intervals results in a widely scattered distribution (Fig 2.7c) which is representative of disorganized atrial activity combined with atrioventricular conduction properties $[49,52,53,57,58,59,60]$.

(a)

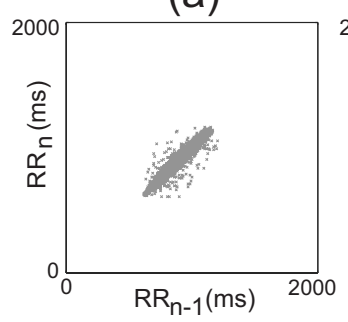

(b)

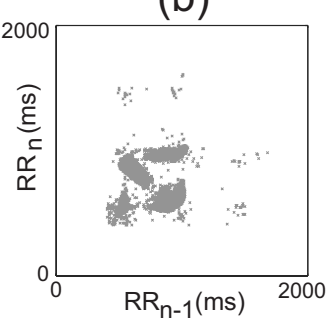

(c)

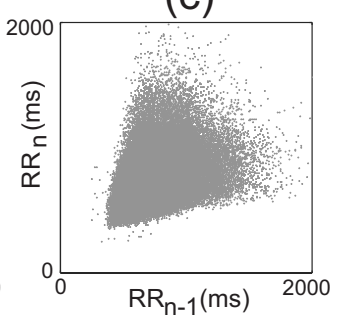

Figure 2.7: Poincaré plots of 24-hour recordings of different patients, during: (a) sinus rhythm, (b) atrial flutter and (c) AF.

By applying Poincaré plot analysis to 24-h Holter recordings of 48 patients with chronic AF, it was suggested that both AV node refractoriness and the degree of concealed AV conduction during AF may show a circadian rhythm, but that these circadian rhythms may be attenuated in patients with heart failure [59]. These findings point to the possibility of obtaining information 
concerning altered autonomic control of the RR intervals in patients with AF (and even for heart failure or other disease) with this simple technique.

In added, patients with $\mathrm{AF}$ and multimodal histograms would exhibit double sector shapes or superimposed scattergrams in the Poincaré plot [60]. These shapes have been evaluated by means of Poincaré plots combined with histographic information [52]. By adding the number of occurrences of RR-interval pairs, a histographic Poincaré plot can be constructed. Presented by van den Berg et al., a bidimensional histographic plot was developed to identify possible clustering of RR intervals [52]. Each point of the histographic Poincaré plot is associated with the number of occurrences of $R R$ interval pairs given by the coordinates $\left(R R_{n-1}, R R_{n}\right)$. Representing the point by gray scale according to the number of the RR occurrences, different clusters can be appreciated as shown in Fig.2.8.

(a)

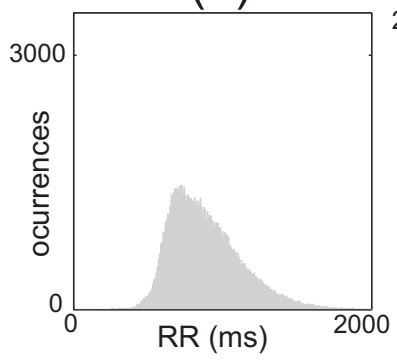

(d)

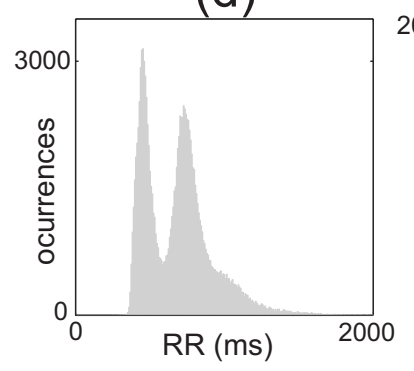

(b)

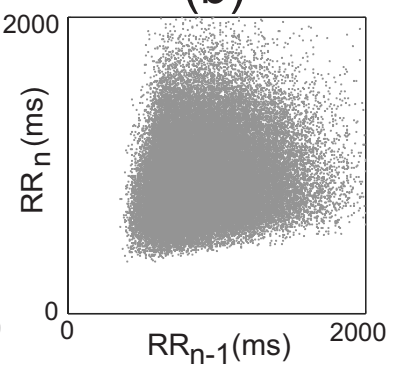

(e)

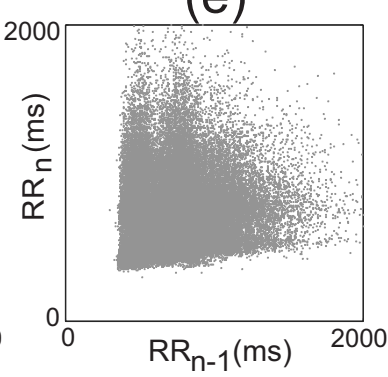

(c)

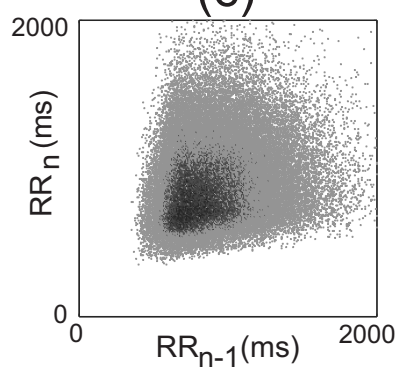

(f)

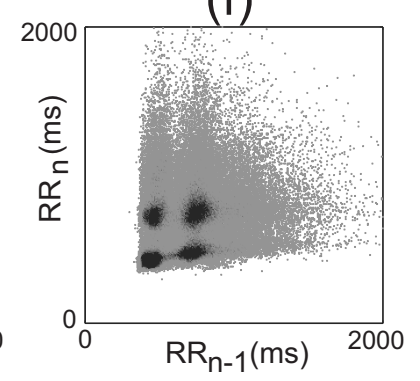

Figure 2.8: 24 hours RR interval histogram (a,d), Poincaré plot (b,e) and histographic Poincaré plot $(\mathrm{c}, \mathrm{f})$ of RR interval series of two patients. Patient 1 (panels a,b and c) presents unimodal RR histogram. Patient 2 (panels d,e and $\mathrm{f}$ ) presents bimodal RR histogram. The histographic information of the Poincaré plot allows the visualization of different number of clusters.

Several considerations should be done about the interpretations of the clusters on the Poincaré plot. Different authors have suggested that the presence of bimodal RR histograms or clusters in the Poincaré plot indicates a 
dual AV nodal physiology [40, 60, 41]. However, the study of RR interval series with the Poincaré analysis does not provide direct evidence of the dual pathways $[60,41]$. It is unclear whether the two pathway properties can be evaluated from the two distributions of the Poincaré plot and the role of the second pathway in the conduction curve needs to be clarified $[3,47,44,45,46]$. In fact, during the development of the current thesis we have attempted to clarify which are the mechanisms producing the existence of more than one $\mathrm{RR}$ interval distribution and the relation between the dual AV nodal pathway physiology and the ventricular response pattern during AF.

Summarizing, the analysis of the Poincaré plot may be useful for evaluating drug effects and AV node modifications on the ventricular response, even if connection between different pathways and $\mathrm{RR}$ interval distributions has not been understood yet. In fact, by means of visual inspection of the histographic Poincaré plots, van der Berg et al. found clustering of RR interval series in $31(47 \%)$ of the 66 patients under study [52]. Moreover, in patients whose $\mathrm{RR}$ intervals were clustered, electrical cardioversion resulted more effective in restoring SR, and also, those patients had a higher likelihood of remaining in SR than their counterparts without well-defined clusters. It was speculated that RR-interval clustering represents a relatively high degree of organization of atrial fibrillatory activity. In the present thesis an in deep evaluation of the potential of Poincaré plots will be described and applied to clarify the role of the atrial fibrillatory activity in the ventricular response.

\subsection{Mathematical Modeling of Atrio-ventricular Conduction Time}

Mathematical models are a repository of knowledge as well as research and teaching tools. Usually, mathematical models are used to explain some behaviors and mechanisms which are not analyzable by means of experimental models. This has been the case in electrophysiology and specifically in the atrioventricular conduction. In fact, mathematical models have been indispensable to clarify some of the characteristic feature of AV nodal function. These include some characteristics present in other cardiac tissues such as conduction slowing of single premature atrial impulses and variable conduction responses to accelerations in rate and some of the specific characteristic features of the AV node such as the Wenckebach periodicity and the concealed conduction:

Wenckebach periodicity is characterized by a conduction pattern where AV nodal conduction time progressively increases from beat to beat until block occurs Fig. 2.9 [61].

Concealed conduction is the effect that produces an atrial beat that fails to get through the His bundle but that partially penetrates in the AV node 


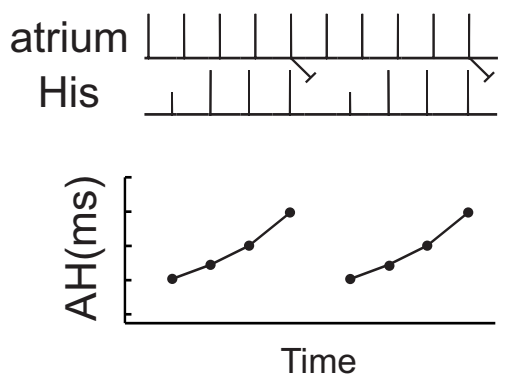

Figure 2.9: Wenckebach Periodicity. A conduction pattern where AV nodal conduction time progressively increases from beat to beat until block occurs.

and consequently alters the AV conduction time at which a subsequent atrial impulse is conducted [62].

We can find in the literature several mathematical models that attempt to reflect some of these characteristics of the AV node from different perspectives and main objectives: 1) modeling of AV time, 2) modeling of RR interval series and 3) modeling of AV node cells and anatomical structures.

\subsubsection{Models of the AV time}

One of the first attempts to model the atrioventicular conduction time was done by Decherd et al. [61]. In that work, Decherd attempted to unmask the role of the AV node in the Wenckebach periodicity. First atrioventricular conduction curves, or recovery conduction curves were plotted and used to estimate the AV conduction time. These diagrams represent the $\mathrm{A}-\mathrm{V}$ conduction time against the preceding V-A interval or the A-A interval. In this preliminary work, the exponential behavior of these curves was suggested, although the AV conduction analysis was based on graphical interpretations.

Some years later, Moe and Abildskov [63], presented a deep analysis of the ventricular response pattern during atrial arrhythmias. In that work, the phenomenon of the concealed conduction, or the effect of non conducted beats in subsequent beats was analyzed in several dog experiments. This descriptive work was the basis of one of the more extensive models presented nine years later by Heethaar et al. [64, 65]. In this work, experiments performed on rats were used to validate the model that, to our knowledge, was the first study in which the AV conduction time was estimated by means an exponential equation formulation [64].

$$
A H_{n}=A+B \cdot \exp \left(-\lambda \cdot A_{n-1} A_{n}\right)
$$

where $A H_{n}$ is the atria to His conduction time (e.g. atrioventricular conduction time) of an atrial beat n, $A_{n-1} A_{n}$ is the time between the last two 
atrial activations and $A, B$ and $\lambda$ are constants.

The mathematical formulation presented in equation 2.1 has been widely used as the basis for later works $[66,67,68,69,70,71,72,73,74]$. Although, in some other works, the usefulness of hyperbolic or pseudo-linear approximations has also been suggested [75, 76]. Usually constants of different mathematical models are fitted from a family of atrioventricular conduction curves like that illustrated in Fig. 2.10.
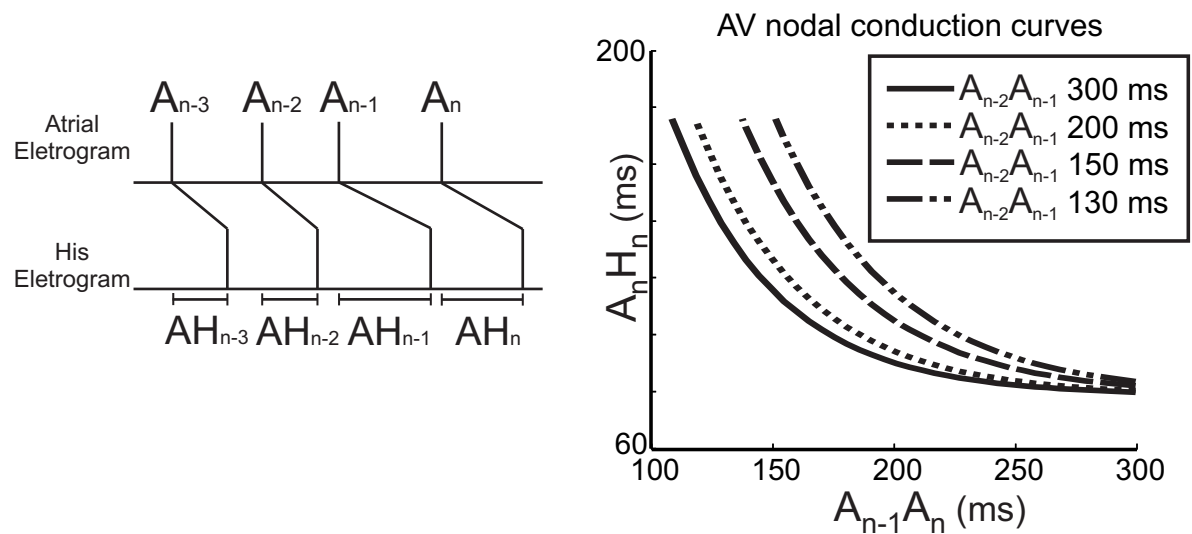

Figure 2.10: Family of AV node conductio curves. Schematic presentation of atrial and ventricular activation periods and the atrioventricular conduction time.

A detailed analysis of all these works may be too extensive. However, some comments on the most influential ones may be helpful to understand the subsequent development of the present thesis. From our point of view, together with the Heethaar et al. [64, 65] model, the model presented in 1991 by Talajic et al. [70] incorporates with high accuracy some of the specific characteristic features of the AV node such as the Wenckebach periodicity and the concealed conduction [65, 70]. Both phenomena, the Wenckebach periodicity and the concealed conduction play an important role in the AV conduction time during supraventricular arrhythmias. However, although a large number of authors have attempt to explain them in detail, specific mechanisms that produce both, Wenckebach periodicity and concealed conduction, has not been precisely elucidated. Nevertheless, the incorporation of both phenomena has been necessary for a precise estimation of the AV conduction time both in experimental models [70] and in human recordings $[72,73]$. 


\subsubsection{Models of the RR interval series}

Modeling of RR interval series is a different approach for studying the ventricular response during AF. The main objective of these works was not to estimate the predicability of its models compared with real experiments. Rather than that, these models are useful to generate RR interval series with similar statistical parameters than those obtained in patients during AF. One of the most influential papers in this research line was presented in 1983 by Cohen et al. [67]. In this case, and in some more recent publications following the same approach $[74,77,78,79]$, authors used Poisson processes to simulate atrial activity during AF and, by using their models, estimate the ventricular response pattern.

One of the most relevant advances to which this research line has contributed is the incorporation of the retrograde ventricle-to-atrial activation. In recent studies by Lian et al.[74, 77, 78, 79], authors illustrated the possibilities of ventricular pacing therapy as a rate control strategy during AF [3].

\subsubsection{Models of AV node cells and anatomical structures}

In a parallel way to all mathematical models based on the so-called atrioventricular conduction curve, some authors have attempted to estimate the atrioventricular conduction time by means of the cellular modeling of cardiac atrioventricular tissues based on Hodkin-Huxley [80] formulation $[81,82,83,84,85,86,87,88,89,90]$. However, most of these studies have been based on ventricular myocyte models due to the lack of specific experiments on ion-channels of AV nodal cells. In addition, the complex morphological structures that anatomically and functionally form the atrioventricular tissues have not been included yet in mathematical models due to the difficulties associated with their analysis and characterization by means of current histological and electrophysiological recording tools. However, recently, great advances have been done in the structural [91, 92, 93] and electrical [94] characterization of these structures at least in a rabbit model.

These novel studies will be very useful to unmask this slick heart structure. However, the main limitation of these detailed models is the large computer time needed for their evaluation. Nowadays, computer capacity limits substantially the possibility to analyze long time periods which may be of great interest for characterizing the atrioventricular behavior during AF. 



\section{Chapter 3}

\section{Poincaré Surface Profiles ${ }^{1}$}

\begin{tabular}{llll}
\hline 3.1 & Introduction & 3.4 & Database \\
3.2 & State of the Art & 3.5 & Results \\
3.3 & Methods & 3.6 & $\begin{array}{l}\text { Discussion and } \\
\text { Conclusion }\end{array}$ \\
& & & Consion \\
\hline
\end{tabular}

The ventricular response (VR) during atrial fibrillation (AF) presents particular characteristics which may play a relevant role in the selection of the most appropriate treatment. Using different ECG signal processing techniques such as RR histogram analysis or histographic Poincaré plots (so-called 3-D Poincaré plots), clusters of RR intervals due to preferential AV node conduction can be observed. However, these methods are limited by the need for visual inspection and subjective interpretation of analysis results.

The objective of this work was to develop a method to automatically detect and quantify preferential clusters of RR intervals. This novel method, the Poincaré Surface Profile (PSP) uses the information of histographic Poincaré plots to filter part of the AV node memory effects. PSP detected all RR populations present in $\mathrm{RR}$ interval histograms in 55 patients with persistent $\mathrm{AF}$; but also $67 \%$ additional RR populations. In addition, a reduction of beat to beat dependencies allowed a more accurate location of RR populations.

This novel Poincaré plot based analysis also allows monitoring of short-term variations of preferential conductions. We illustrate the capability of this shorttime monitoring technique to evaluate the effects of rate control drugs on each preferential conduction.

\footnotetext{
${ }^{1}$ Chapter based on the manuscript: Andreu M. Climent, M. Salud Guillem, Daniela Husser, Francisco Castells, Jose Millet and Andreas Bollmann. Poincaré Surface Profiles of RR intervals. A Novel Noninvasive Method for the Evaluation of Preferential AV nodal Conduction during Atrial Fibrillation. IEEE Trans Biomed Eng. 56(2):433-42. 2009
} 


\subsection{Introduction}

Atrial fibrillation (AF) is characterized by an unorganized electrical activity of the atria. This is associated with irregular ventricular responses (VR) with typically shorter RR intervals than during normal sinus rhythm [3].

Normally, in the clinical practice only mean ventricular rate and its variability are used to describe $\mathrm{VR}$ and to evaluate treatment effects. However, the VR during AF presents additional particular characteristics that can be analyzed by different signal processing techniques [53, 49]. By constructing histograms of $\mathrm{RR}$ intervals during $\mathrm{AF}$, different populations of more probable RR intervals can be found [95, 40, 41]. Additionally, different clusters of $\mathrm{RR}$ interval sequences can be made visible in Poincaré plots combined with histographic information $[60,52,96]$.

Both methods, histograms and Poincaré plots, have shown their potential usefulness in clinical decision-making [52, 97, 43, 98, 99, 42, 100]. For instance, the presence of bimodal histograms has been shown to be associated with success of atrio-ventricular (AV) node modification reducing mean RR interval (e.g. ablation of the slow AV node pathway) [96, 43, 98]. Furthermore, clustering in bidimensional histographic scattergrams (so-called 3D-plot, 3-D Poincaré plot or 3-D Lorenz plot) has predicted the effectiveness of electrical cardioversion [52]. Taken together, identification and quantification of preferential AV nodal conductions may play an important role in choosing the appropriate treatment for a given patient. However, AV nodal conduction mechanisms are poorly understood and difficult to quantify in the clinical setting [3].

Current AF management guidelines mention that there are fundamentally two ways to manage this rhythm disorder: to restore and maintain sinus rhythm (SR) or to allow AF to continue and ensure that ventricular rate is controlled [4] both of which seem to have similar outcomes [5]. The rate control strategy of AF deals with efforts to utilize and adjust the propagation properties of the AV node [3]. Nevertheless, treatment effects on the AV nodal conduction properties and consequently VR are not easy to assess.

The objective of this work was to develop a method to automatically detect preferential AV node conductions expressed as RR interval sequences both in long- and short-term ECG recordings, and hence with the capability to monitor short-term variations. Moreover, it was the aim to improve the sensitivity for detecting different RR populations over existent techniques such as RR interval histogram analysis.

In this work, novel techniques for automatic characterization of the VR during AF, i.e. the Poincaré Surface and the Poincaré Surface Profile are introduced. In addition, the ability of this technique to monitor short-term variations of preferential conduction and to monitor rate control treatment is illustrated. 


\subsection{State of the Art}

Ventricular rate during $\mathrm{AF}$ is analyzed by measuring and visualizing the time between two ventricular activations ( $R R$ intervals). Only normally conducted beats are used for further analysis, whereas wide QRS complexes and artifacts are excluded. Current, related methods to analyze RR interval distributions include (A) RR interval histograms, (B) Poincaré plots, (C) histographic Poincaré plots [53], which are described below.

\subsubsection{RR Interval Histograms}

In long $\mathrm{RR}$ series the $\mathrm{RR}$ interval histogram can be regarded as an approximation of the probability density function (pdf), where the parameter corresponding to the $\mathrm{RR}$ interval is discretized in several $\mathrm{RR}$ interval categories. Accordingly, the histogram represents the number of occurrences given at each category. The maximum resolution that can be achieved is the sampling interval, although a reliable histogram analysis would require in this case long ECG recordings. Therefore, a compromise between the number of $\mathrm{RR}$ intervals needed for the construction of RR histograms and accuracy in $\mathrm{RR}$ interval estimation should be established in order to choose an appropriate histogram resolution.

In order to reduce the statistical variability of the histogram due to the limited series of RR intervals, histogram smoothing can be performed using a low pass filter. As an example, a filtering technique based on applying repeatedly a sliding average window has been proposed in [97]. A special form of analyzing RR histograms by constructing RR interval histograms according to mean heartrate are the so-called heart rate stratified histograms (HRSH) [95, 40].

By constructing histograms of RR interval series during AF, uni-, bi-, or multimodal RR distribution patterns can be found [40, 96, 43, 42, 100, 101]. It has been suggested that a bimodal $\mathrm{RR}$ interval distribution during chronic $\mathrm{AF}$ is associated with the presence of dual AV nodal physiology. It predicts a better outcome of radiofrequency ( $\mathrm{RF}$ ) ablation of the posterior atrionodal input (slow pathway) [40, 100]. However, the bimodal RR interval distribution analysis is not always related with the presence of dual AV nodal pathway physiology, and a unimodal histogram pattern may still be associated with dual AV nodal pathways [41, 47]. Nevertheless, modifications of the AV node in patients presenting with bimodal RR interval distributions were more effective in reducing the heart rate than in patients with an unimodal pattern $[43,98]$.

\subsubsection{Poincaré Plots}

In RR interval scattergrams, so-called Poincaré plots (PP) or Lorenz Plots, each $\mathrm{RR}$ interval is presented in the vertical axes and the immediately preceding 
$\mathrm{RR}$ interval in the horizontal axis $[54,102,58,56]$. In this representation the Poincaré plot is set to 1 at each pair (RRn-1,RRn):

$$
P S P(x, y)= \begin{cases}1 & \text { if } \exists R R_{n-1}=x, R R_{n}=y \quad 2 \leq n \leq N \\ 0 & \text { otherwise }\end{cases}
$$

where $\mathrm{N}$ is the total number of $\mathrm{RR}$ intervals. This representation produces a scattergram of couples of $\mathrm{RR}$ intervals which allows the measurement of beat to beat dependencies. Analogously to the histogram, the maximum resolution of Poincaré plots corresponds to the sampling interval. An example of an RR interval scattergram during AF is illustrated in Fig. 3.1.a.

\subsubsection{Histographic Poincaré plot}

In histographic Poincaré plots, so-called 3D-plots [52], the number of occurrences of RR interval pairs at each pixel of the Poincaré plot is added as the height in a third axis. The value of each pixel of the histographic Poincaré plot is equal to the number of occurrences of RR interval pairs given by the $(\mathrm{x}, \mathrm{y})$ coordinates of the pixel. If long ECG recordings are to be analyzed, a high number of occurrences may be present at each pair $(\mathrm{x}, \mathrm{y})$, which are disregarded in the Poincaré plot. The histographic Poincaré plot adds this information, which can be useful to analyze the probability of occurrence of a $\mathrm{RR}$ interval conditioned to the previous RR interval (Fig 3.1.b.).

In histographic Poincaré plots of patients with AF, clusters of more probable RR intervals were observed [8]. This finding supports the hypothesis that ventricular rhythm during AF may not be completely random. In this study, electrical cardioversion was shown to be more successful in patients whose histographic Poincaré plots were clustered, resulting in a higher rate of restoration of sinus rate and higher likelihood of remaining in sinus rhythm than their counterparts without well-defined clusters.

\subsubsection{Detection of Preferential AV Node Conduction}

Preferential AV node conduction is usually detected from the RR interval histogram. As mentioned before, either unimodal or multimodal distributions can be found during AF. The correct detection of preferential AV node conduction can be useful to characterize and understand the response of the AV node. However, there are situations where the analysis of the conventional histogram can lead to wrong conclusions:

One situation that is necessarily to take into account is that a multimodal histogram constructed from a long-time recording does not mean by itself that different preferential AV conductions exist. In fact, two different situations can result in a bimodal histogram: either (1) two clusters are present simultaneously or (2) two clusters are present at different times, e.g. corresponding to resting 
and active periods with different heart rates. These two situations could be distinguished by looking at the out of diagonal clusters of the Poincaré plot. The detection of transition clusters outside the diagonal in the Poincaré plot may indicate frequent transitions between more than one preferential atrioventricular conduction intervals during a specific time under study.

Another important consideration for the analysis of preferential AV nodal conduction by means of conventional RR histograms is that the AV node presents significant beat to beat dependencies, which may distort the measurements. In order to illustrate how the beat to beat dependencies of the AV node may influence the analysis of the histogram, let us consider the histographic Poincaré plot in Fig. 3.1.b.

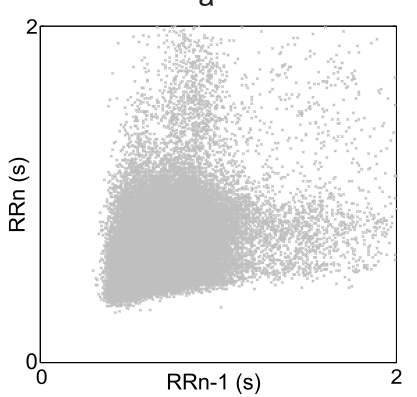

c

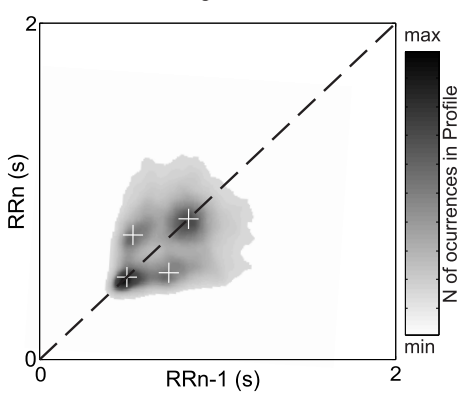

b

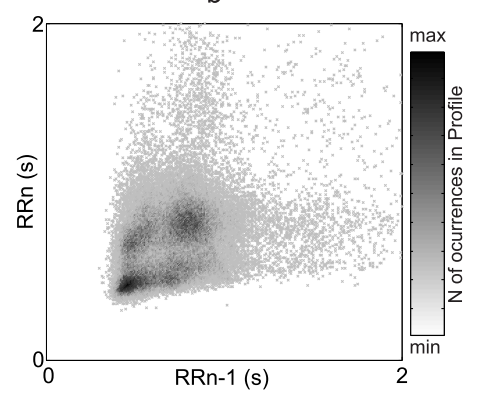

d

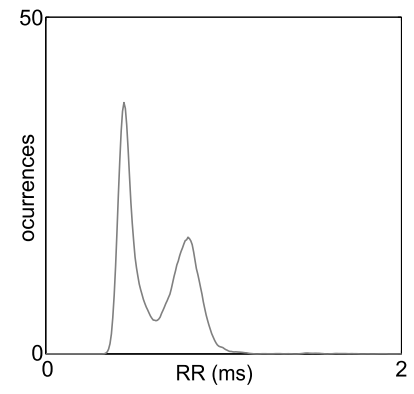

Figure 3.1: In Panel (a), a Poincaré plot with RR intervals obtained during 24 hours is depicted. In panel (b), the same Poincaré plot is shown, but the number of occurrences of RR pairs is color-coded. The darkest points represent the highest number of occurrences. In panel (c), the Poincaré Surface is illustrated, with white crosses indicating local maxima that are automatically detected. From the dashed line (Panel (c)) the Poincaré Surface Profile (PSP) is constructed that is illustrated in panel (d). 
In this patient, four RR interval populations have been identified: (1) consecutive short RR intervals, (2) consecutive long RR intervals, (3) alternate short and long RR intervals and (4) alternate long and short RR intervals. In an RR interval histogram analysis a bimodal distribution would have been represented. In addition, by observing the histographic Poincaré plot, a displacement of the RR interval can be appreciated when a transition occurs. In fact, when a short RR interval occurs after a long RR interval, it is slightly longer in comparison to consecutive short RR intervals. Analogously, when a long RR interval occurs after a short RR interval, it is slightly shorter in comparison to consecutive long intervals. This memory property of the AV node is reflected in the RR interval histogram, so that the clusters corresponding to each of the preferential AV node conductions appear shifted and closer to each other, which introduces the following problems: (1) errors in the estimation of the RR intervals and (2) if the clusters become too close they may be practically joined into a single cluster and it may be difficult to identify a multimodal distribution.

In the following section we will present novel methods to overcome these problems and enhance the detection of preferential AV node conductions. The suggested techniques are fully automated, and therefore independent of visual inspection and subjective data interpretation.

\subsection{Methods}

In this section different signal processing techniques are presented aimed at the characterization of the histographic Poincaré plot. Section A describes methods for cluster identification, such as definition of the number of clusters or evaluation of the position and number of occurrences of RR interval pairs in each cluster. In section $B$ a method to detect preferential AV nodal conductions is presented. This method, namely the Poincaré Surface Profile (PSP), employs the histographic Poincaré plot to construct a filtered version of the RR interval histogram where the RR intervals involved during a change in the AV node response are disregarded. As a result, an RR interval distribution more independent of the memory properties of the AV node is obtained, which overcomes the limitations of conventional histogram analysis and allows a more accurate and reliable detection of preferential RR interval. In section $\mathrm{C}$ an application of the PSP for rate control monitoring treatment is described.

\subsubsection{Poincaré Surface and Cluster Identification}

The problem of the variability due to limited $R R$ interval series presented in section 3.2 is emphasized in the case of the histographic Poincaré plot. This is a critical factor if a Poincaré plot is to be constructed from a small number of RR intervals, since clusters can not always be easily identified. In order to 
emphasize clusters, reduce the variability and increase the accuracy in cluster location, a two-dimensional low-pass filter can be applied to the histographic Poincaré plot. This processing results in a smooth surface plot or Poincaré Surface (PS). Due to the properties of the surface described by RR interval populations during AF, which has been modeled as a bidimensional Gaussian distribution [67], we propose a rotationally symmetric Gaussian lowpass filter with standard deviation $\sigma$ to smooth the histographic Poincaré plot:

$$
h\left(\tau_{1}, \tau_{2}\right)=\frac{1}{2 \pi \sigma^{2}} e^{\frac{\tau_{1}^{2}+\tau_{2}^{2}}{2 \sigma^{2}}}
$$

The PS is the result of filtering the histographic Poincaré plot with the two dimensional filter $h\left(\tau_{1}, \tau_{2}\right)$. Fig. 3.1.b shows a histographic Poincaré Plot in which an irregular surface with a high amount of local maxima can be observed. After applying the Gaussian filter in (2) to the histographic Poincaré plot, a smoother surface is obtained as depicted in Fig. 3.1.c.

Detection of clusters in the PS can be performed as follows: a local maximum on the Poincaré surface is defined at the pair $\left(x_{\max }, y_{\max }\right)$ if

$$
P S(x, y)<P S\left(x_{\max }, y_{\max }\right), \quad\left\|\left(x-x_{\max }\right),\left(y-y_{\max }\right)\right\|<\epsilon
$$

where $\epsilon$ is a parameter that can be used to avoid the detection of neighbor local maxima that indeed belong to the same cluster. Automatic detection of clusters is illustrated in Fig. 3.1.c.

\subsubsection{Poincaré Surface Profile}

The PSP is defined as the diagonal of the Poincaré Surface:

$$
P S P(x)=P S(x, x)
$$

This can be interpreted as filtering the RR interval histogram by considering only RR intervals that were preceded by beats with approximately the same RR interval and using bidimensional information of neighboring RR interval couples thanks to the previous smoothing process. The PSP corresponding to the PS in Fig. 3.1.c (dashed line) is depicted in Fig. 3.1.d.

An algorithm to identify significant peaks on the PSP can automatically detect and characterize different RR populations on the PSP. A peak can be considered as significant if it fulfils two criteria: (1) be a local maximum and (2) have a higher amplitude than a defined threshold, e.g. an automatic threshold can be computed as a percentage of the maximum amplitude. All peaks whose number of occurrences is lower than this threshold are considered noise. Finally, if two peaks are closer than a specific distance $(\epsilon)$ the peak with the lowest amplitude is also discarded. 
Peaks detected in the PSP are labeled as 1, 2, 3, etc. according to the position on the RR cluster in ascending order. In case that three peaks are detected in the PSP the distance between peaks 2 and 1 (D2-1) and the distance between peaks 3 and 2 (D3-2) are computed and compared.

\subsubsection{Short Time Variations on the Ventricular Response}

Short time variations in the ventricular response can be analyzed by computing a 30 -minute PSP every 15 minutes, with $50 \%$ overlapping (i.e. for a Holter recording of 24 hours, 95 PSP from overlapping sequential segments can be generated), so that the behavior of each cluster along the day can be monitored.

\subsubsection{Statistical Analysis}

Data are presented as mean +/- standard deviation. Pearson correlation analysis was performed to analyze the possible association between peaks obtained with the PSP. The paired Student's t test was performed to compare the position of peaks detected by PSP and the position of peaks detected by the histogram.

Chi-square test was used to compare the number of peaks detected by the PSP method with the number of peaks detected by the histogram. A p value $<0.05$ was considered statistically significant.

\subsection{Database and Preprocessing}

65 consecutive patients (52 male, 13 female, mean age $66 \pm 10$ years) with persistent AF (AF duration 18 \pm 20 months) were included in this study. For every patient, a clinically indicated ambulatory Holter ECGs of approximately 24 hours was recorded. Holter recordings with a high degree of noise or with a percentage of ventricular ectopic beats and aberrant beats $>20 \%$ were excluded $(\mathrm{N}=10)$. Three Holter recordings were available for one patient: two Holters during baseline and one Holter ECG during rate control treatment with beta blockers.

Holter ECGs were acquired during usual daily activities using a CardioMem CM 3000 recorder (Fa. Getemed, Teltow, Germany) with a sampling rate of $128 \mathrm{~Hz}$. QRS complexes were detected using Getemed software. Results of automatic analysis were visually inspected and each error in the detection or labeling was corrected manually. Duration of each RR interval and classification of all beats were exported for off-line analysis using Matlab 7 (The Mathworks, Inc., Natick, USA). 


\subsection{Results}

\subsubsection{Poincaré Surface and Cluster Identification}

The irregularity of $\mathrm{RR}$ intervals during $\mathrm{AF}$ resulted in a wide scattered distribution on the Poincaré plot. However, histographic Poincaré plots showed different clusters of more probable couples of RR intervals.

After testing different resolutions, we decided to set it to the maximum of our ECG recorder system, with a sampling frequency of $128 \mathrm{~Hz}$ (i.e. the resolution was $7.8125 \mathrm{~ms}$ ). In order to construct a PS a rotationally symmetric Gaussian lowpass filter with a standard deviation of $25 \mathrm{~ms}$ was used.

In Fig. 3.2.a., typical PS types of four patients with AF are depicted. These four patients presented similar $R R$ mean and $R R$ variability (table 3.1 ). The standard Poincaré plot of these four patients did not present clear differences but a wide and dispersed VR. However, by adding the number of occurrences of couples of RR intervals, the histographic Poincaré plot unmasked differences of the VR organization degree. The construction of PS emphasized these differences. Patient 1 presented one wide cluster on the PS. Patient 2 presented four clearly separated clusters. Patient 3 presented four overlapping clusters. Patient 4 presented multiple clusters.

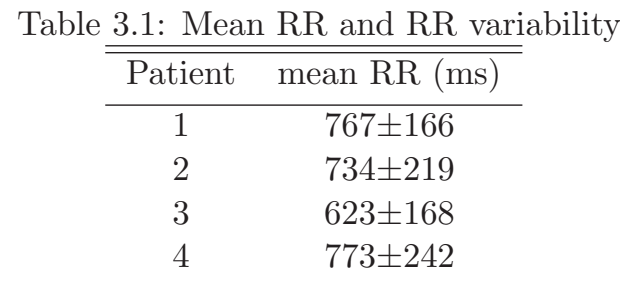

In order to characterize the PS automatically, a minimum distance between peaks of $50 \mathrm{~ms}$, epsilon in equation 3, was chosen as optimal to define a local maximum. Position and number of occurrences of detected clusters in the four patients are shown in table 3.2.

\subsubsection{Poincaré Surface Profile. Comparison with Histograms}

In order to analyze possible improvement of the PSP over conventional RR interval histogram for analyzing $\mathrm{RR}$ interval distributions, the number of $\mathrm{RR}$ interval clusters, their location and number of occurrences were evaluated by applying both methods in all Holter ECG recordings. Conventional RR interval histograms were constructed with the same resolution used for Poincaré plots. They were filtered with the smoothing procedure presented in [97]. It consisted of the application of a moving average filter until the minimum distance between 

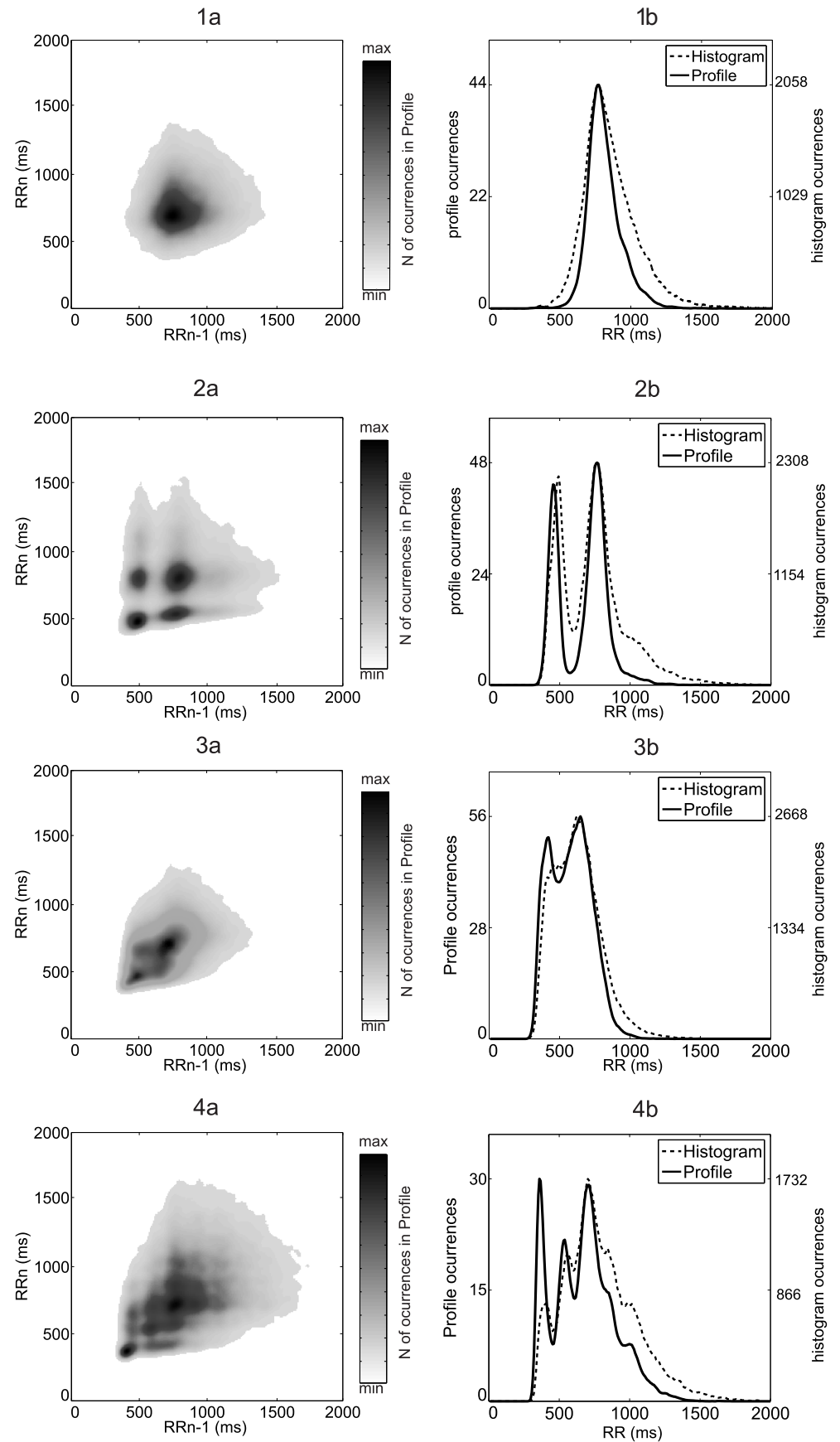

Figure 3.2: 24-hour Poincaré Surface (panels a) and RR interval histogram (dashed line in panels b) and Poincaré Plot Profile (continuous line in panels b) of four patients with four different VR patterns. 
Table 3.2: Positions of detected clusters on the local maximum of the cluster for four patients. Position of a cluster is defined by its positions in the horizontal axis (RRn-1) in ms. and the position in the vertical axis (RRn) in ms.

\begin{tabular}{cc|cc|cc|cc}
\hline \hline \multicolumn{2}{c|}{ Patient 1 } & \multicolumn{2}{|c|}{ Patient 2 } & \multicolumn{2}{c|}{ Patient 3 } & \multicolumn{2}{c}{ Patient 4 } \\
\hline RRn-1 & RRn & RRn-1 & RRn & RRn-1 & RRn & RRn-1 & RRn \\
700 & 677 & 459 & 467 & 418 & 424 & 375 & 359 \\
& & 756 & 768 & 651 & 646 & 531 & 531 \\
& & 749 & 488 & 611 & 462 & 711 & 703 \\
& 493 & 757 & 464 & 594 & 555 & 688 \\
& & & & & 703 & 563 \\
& & & & & 836 & 711 \\
& & & & & 711 & 836 \\
\hline
\end{tabular}

two local maxima was higher than 50 ms. Peaks detected in the conventional RR interval histogram were labeled as 1, 2, 3, etc. according to the closest peak found in the PSP.

Significant peaks were detected in both the PSPs and histograms by using the method previously described in the section 3.3.B.. The threshold used for the definition of significance was $10 \%$ of the maximum peak and $50 \mathrm{~ms}$ was chosen as the minimum distance between peaks.

In patient 1 , both the histogram and the PSP showed only one cluster, or a wide unimodal pattern. In patient 2, both the histogram and the PSP depicted a bimodal pattern, where most probable RR interval clusters showed similar number of occurrences. In the PSP, the valley between the two peaks was emphasized. Two peaks were automatically detected by means of both algorithms. In patient 3, visual inspection of the histogram does not allow to distinguish between one or two clusters whereas the PSP showed two clearly defined peaks. By means of the automatic algorithm described in section 3.3.b., one cluster was detected in the histogram whereas in the PSP two peaks were detected. Patient 4 showed an RR interval histogram with one dominant cluster and sawtooth-like tails. Two peaks were automatically estimated by using the detection algorithm to the histogram. In this case, the PSP showed three separated clusters and two pronounced slope changes in long RR intervals. The first three RR peaks were automatically detected.

In Table 3.3 the distributions of patients according to the number of peaks detected by means of PSP and conventional RR interval histograms are summarized. All RR populations found in the RR interval histogram were detected by means of the PSP. In summary, 61 RR peaks were detected in the conventional histograms whereas $102 \mathrm{RR}$ peaks were detected in the PSP.

Fig. 3.3 shows the difference between the positions of each peak detected in the PSP and the position of each peak detected by means of the conventional histogram when more than one peak was detected in the PSP. $96 \%$ of type 1 
Table 3.3: Distribution of patients according to the number of peaks detected by means of PSP and RR interval histogram.

\begin{tabular}{ccc}
\hline \hline & PSP & Histogram \\
\hline Patients with 1 RR population & 19 & 30 \\
Patients with 2 RR populations & 25 & 22 \\
Patients with 3 RR populations & 11 & 3 \\
\hline
\end{tabular}

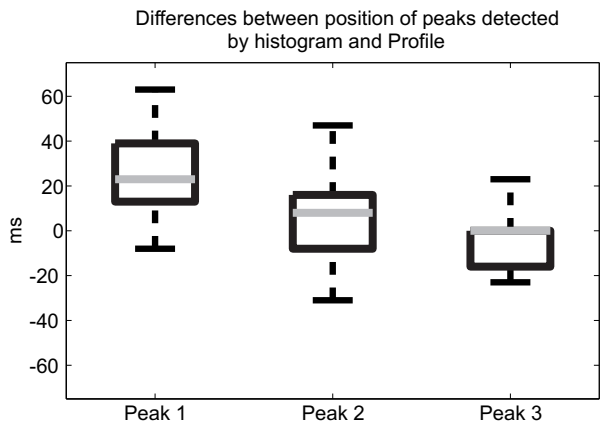

Figure 3.3: Boxplots of the difference between the positions of each peak detected by means of RR histograms or PSP. Boxes have lines at the first quartile (bottom), median (gray line), and third quartile values (top). The whiskers are lines extending from each end of the box to show the extent of the rest of the data.

peaks were found at higher positions in the histogram compared to the PSP, with a mean difference of $26 \pm 19 \mathrm{~ms}(\mathrm{p}<0.01)$. Type 2 peaks were found in the PSP at either lower or higher locations compared to the histogram, a mean difference of $6 \pm 18 \mathrm{~ms}(\mathrm{p}=0.05)$ indicates a small but significant higher positions in the histogram compared to the PSP. Type 3 peaks were found predominantly $(86 \%)$ at equal or lower positions in the histogram than in the PSP, with a mean difference of $2 \pm 15 \mathrm{~ms}$ ( $\mathrm{p}=$ n.s.).

\subsubsection{Poincaré Surface Profile and Transition Clusters in Poincaré Surface}

Joint information of the number of peaks found in the PSP and the existence of transition clusters in PS is summarized in table 3.4. In 18 of the 19 patients with one peak in the PSP no transitional clusters were found. One patient with one peak in the PSP presented three clusters in the PS, one in the diagonal and two in symmetrical positions around it. In 20 of the 25 patients with two peaks in the PSP, 2 transition clusters were detected. In 1 of the 25 patients one transition cluster was detected. In 2 of the 25 patients 3 transition clusters 
were detected. In 2 of the 25 patients no transition clusters were detected. In all 11 patients with three peaks in the PSP more than 2 transition clusters were detected.

Table 3.4: Distribution of patients according to the number of peaks detected by means of PSP and the transition clusters detected in the Poincaré Surface.

\begin{tabular}{ccl}
\hline \hline NPeaks in the PSP & & Number of patients \\
\hline 1 & 18 & without transition clusters \\
& 1 & with transition clusters \\
\hline 2 & 2 & without transition clusters \\
& 23 & with transition clusters \\
\hline 3 & 0 & without transition clusters \\
& 11 & with transition clusters \\
\hline
\end{tabular}

In the 23 patients in which two peaks were found in the PSP and at least one transition cluster was detected in the PS, mean position of peaks 1 and 2 were $434 \pm 85 \mathrm{~ms}$ and $684 \pm 92 \mathrm{~ms}$ respectively.

In the 11 patients with three peaks mean position of peaks 1,2 and 3 were $471 \pm 105 \mathrm{~ms}, 717 \pm 161 \mathrm{~ms}$ and $947 \pm 227 \mathrm{~ms}$. Pairwise correlation among the location of the three populations was higher than $0.91(\mathrm{p}<0.01)$. The mean distance between peaks 2 and 1 (D2-1) was $246 \pm 68 \mathrm{~ms}$ and the mean distance between peaks 3 and 2 (D3-2) was $230 \pm 78 \mathrm{~ms}(\mathrm{p}=\mathrm{n} . \mathrm{s})$. The correlation between D2-1 and D3-2 was $\mathrm{R}=0.97(\mathrm{p}<0.01)$. The mean absolute difference between D3-2 and D2-1 was $15 \pm 14$ ms.

\subsubsection{Illustration of Short Time VR Variations vs Variation of Preferential Conduction}

Short-time variations in the PSP over the 24-hour Holter recording time are depicted in Fig. 3.4. In each panel, short time variations of the amplitude of the PSP in intervals of 30 minutes with an overlap of 15 minutes are represented. RR clusters were detected in each PSP by using the method described in section 3.3. Panels a, b, c and d correspond to patients 1, 2, 3 and 4 in Fig. 3.2 respectively.

In panel (a) a fairly constant unimodal pattern was present during the whole day. The mean position of the detected peak in each PSP was $697 \pm 28 \mathrm{~ms}$.

In panel (b) two separated RR clusters at $450 \pm 32 \mathrm{~ms}$ and $738 \pm 34 \mathrm{~ms}$ were detected during most part of the day. A cluster centred at $738 \mathrm{~ms}$ was detected during the whole recording with similar number of occurrences. On the other hand, the number of occurrences in the cluster situated around $450 \mathrm{~ms}$ changed during the day. It had a high number of occurrences concentrated in the peak between 9:15 to 19:45, the number of occurrences decreased during the rest of the day and it was not detected from 23:00 to 5:00. 
In patient from panel (c), two peaks were detected in the 24 hour PSP (Fig. 3.2) at $426 \mathrm{~ms}$ and $649 \mathrm{~ms}$. By evaluating short-time variations by visual inspection a higher degree of dispersion in the PSPs could be observed and a lower definition of transitions between both detected clusters than in patient 2. By means of the automatic characterization two clusters were discriminated, one at $406 \pm 25 \mathrm{~ms}$ and the other at $641 \pm 80 \mathrm{~ms}$. The cluster centred around 641 ms was detected during the whole day although with a considerable variation in the position ( $80 \mathrm{~ms}$ ). The RR interval centred around $406 \mathrm{~ms}$ presented a high number of occurrences between 12:15 to 13:00, 13:45 to 18:00 and 8:00 to 10:15.

In panel $(\mathrm{d})$ corresponding to patient 4 , three separated clusters at $370 \pm 18$ $\mathrm{ms}, 543 \pm 22 \mathrm{~ms}$ and $707 \pm 32 \mathrm{~ms}$ were detected. The cluster centred at $707 \mathrm{~ms}$ was detected during the whole day without large variations in the number of occurrences. The cluster centred at $543 \mathrm{~ms}$ was detected continuously from 10:15 to 13:45 and from 5:45 until the end of the Holter and it was intermittently detected during the rest of the day. The cluster centered at 370 ms was detected from 10:15 to $12: 45$, from 13:30 to 14:15 and from 8:30 to the end of the recording. This cluster with short RR intervals had the higher number of occurrences. Notice that three clusters were simultaneously present from 10:30 to $12: 45$, from $13: 30$ to $14: 15$ and from 8:30 to the end of the recording.

\subsubsection{Illustration of Drug Effects}

In order to illustrate the utility of monitoring short time variations in the PSP to evaluate rate control treatments, a second baseline recording and a recording during beta blockers treatment of patient 2 of the Fig. 3.2 and 3.4 are depicted in Fig. 3.5. In panel 1 the reproducibility of the method is evaluated with a second Holter recording acquired seven days later. In panel 1.a. the PS shows four clusters with similar number of occurrences; two in the diagonal and two in the transition positions. In panel 1.b. two separated RR clusters at $463 \pm 26 \mathrm{~ms}$ and $763 \pm 29 \mathrm{~ms}$ are detected during most part of the day. The cluster centred at $763 \mathrm{~ms}$ was detected during the whole day. The cluster centred at $463 \mathrm{~ms}$ was detected from the beginning of the recording until 22:00 and from 6:00 until the end of the recording.

In panel 2 of Fig. 3.5 the effects of the beta blockers treatment is illustrated. Due to the negative dromotropic effect of the beta blockers, the mean RR interval increased from $685 \pm 217 \mathrm{~ms}$ in the first Holter (Fig. 3.4 panel 2) to $788 \pm 205 \mathrm{~ms}$ in the third Holter (Fig. 3.5 panel 2). In panel 2.a. is depicted the PS. Four clusters were detected, two in the diagonal and two in the transition positions. In this case the number of occurrences in the long RR intervals cluster in the diagonal was higher than in the other three clusters. In the short time variations in the PSP depicted in panel 2.b. two separated RR clusters were detected at $465 \pm 10 \mathrm{~ms}$ and $716 \pm 20 \mathrm{~ms}$. The cluster centred at 

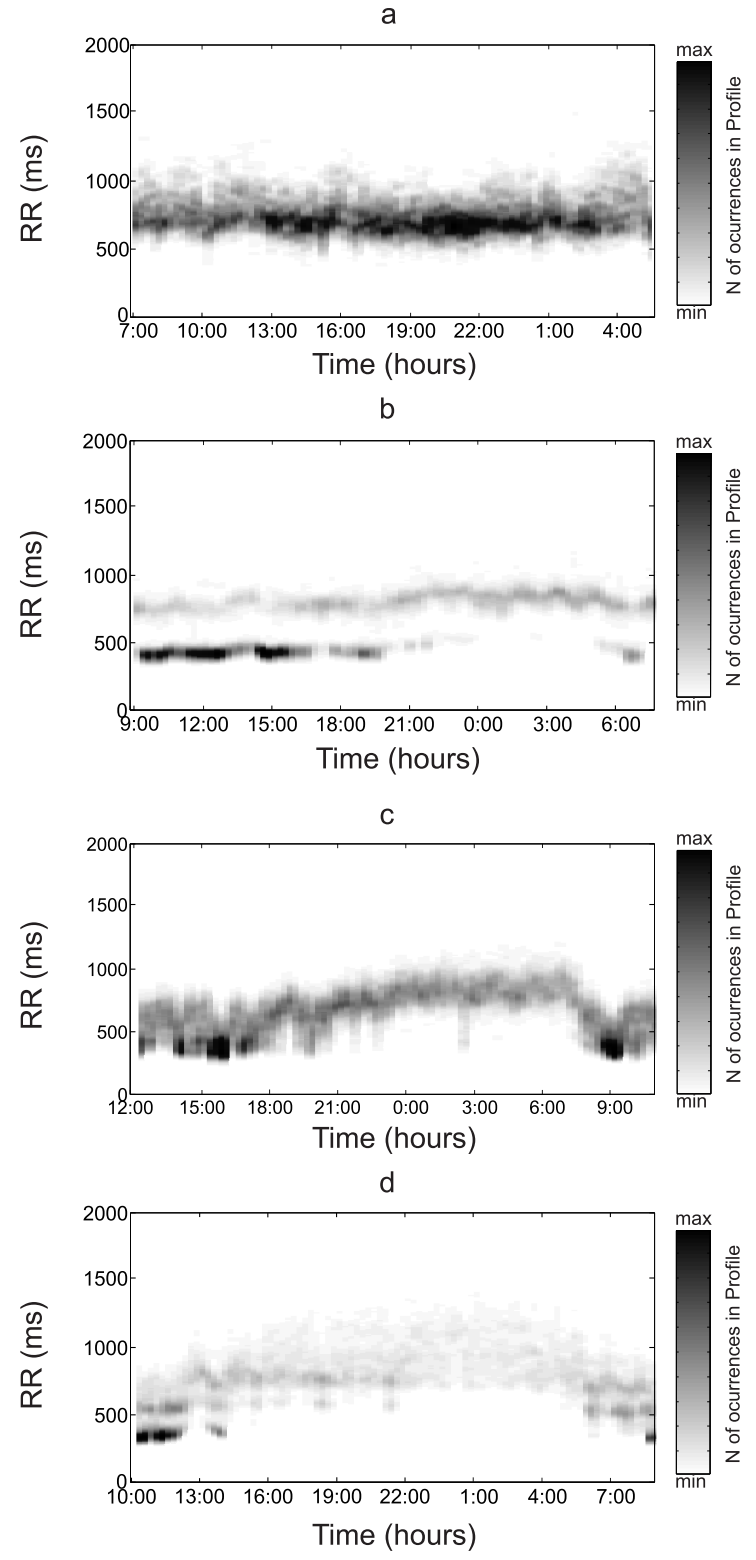

Figure 3.4: Short-time variations of the Poincaré Surface Profile (PSP). One PSP calculated from a time lag of 30 minute every 15 minutes. Panels a, b, c and d represent the 24 hour PSP variation of patients 1, 2, 3 and 4 of Fig. 3.2 respectively. Horizontal axes correspond to the time interval available in each Holter recording. 
716 ms was detected during the whole day with a higher number of occurrences than in the baseline Holters. In this Holter the cluster centred at $465 \mathrm{~ms}$ was detected intermittently from 12:00 to 19:00.
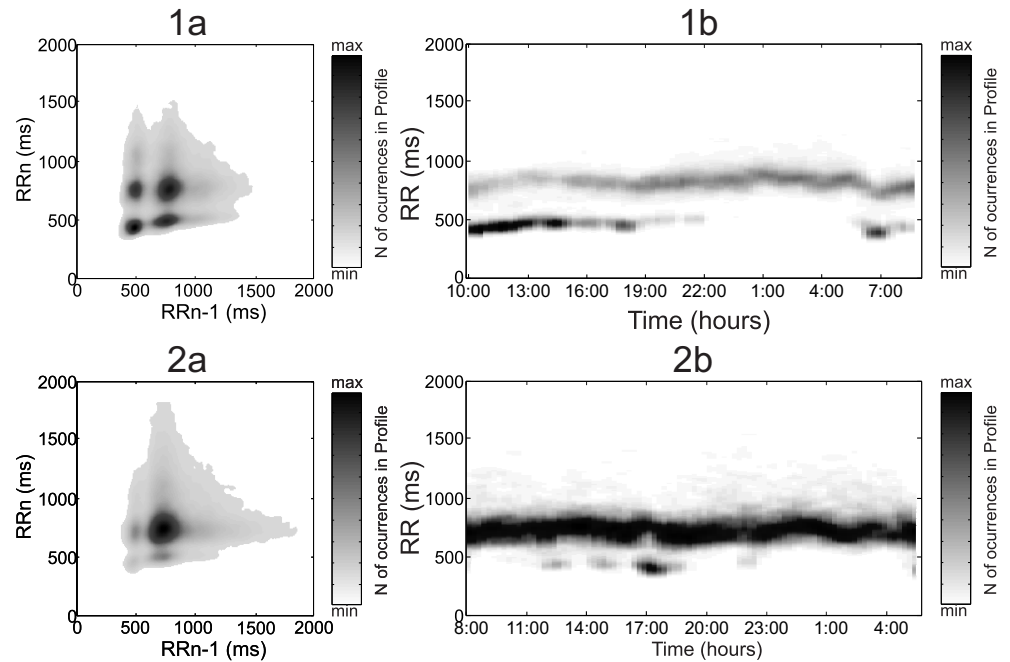

Figure 3.5: Panel 1 represents a second baseline Holter of patient 2 of Figures 3.2 and 3.4. In panel 2 a Holter of the same patient during rate control treatment with beta blockers is presented. In panels 1.a. and 2.a. Poincaré Surfaces are depicted. Panels 1.b and 2.b show short-time variations in the PS.

\subsection{Discussion and Conclusion}

In this work, different signal processing techniques have been proposed to identify and quantify preferential AV node conductions from ECG recordings during AF.

We have presented an automatic method for the characterization of clustering in a histographic Poincaré plot during AF. We have introduced the Poincaré Surface plot for reducing the dispersion of RR populations, allowing a robust and reproducible cluster detection. This is the first time that an automatic technique to detect clusters in the Poincaré plot is presented. The ability of PS to identify clusters has been illustrated with four patients (Fig. 3.2.a. and table 3.2.). These patients presented four different PS patterns, although mean RR interval and RR variability during 24 hours did not present significant differences (table 3.2).

The detection of transition clusters has been presented as an indicator of the presence of multiple simultaneous preferential AV nodal conductions. Nevertheless, position of transition clusters is influenced by the memory effects 
of the AV node (i.e. Wenckebach phenomenon), and consequently their locations do not give an accurate position of the preferential $\mathrm{RR}$ interval. These effects depend mainly on preceding $R R$ intervals, which alter the atrioventricular conduction time and modify the RR interval. The importance of transition clusters should not be underestimated but as they are affected by AV node memory effects, they should not be used for the estimation of the position of preferential AV nodal conduction.

Once transition clusters are detected in the Poincaré Surface, we propose the Poincaré Surface Profile technique for improved detection of preferential atrioventricular conductions during AF over conventional $\mathrm{RR}$ interval histograms. By using the PSP method transitions between preferential AV nodal conductions are not considered because they lie outside the identity line in the Poincaré plot, consequently reducing blurring effects of the memory of the AV node. This allows a more accurate detection of preferential RR interval conductions, both in the number of clusters and in the position of the clusters. Results depicted in Fig. 3.3 support these considerations. Type 1 peaks correspond to shorter RR intervals in the PSP than in the histogram in most cases. Transitions between short and intermediate RR populations only increase the superior tail of the shortest RR population in the histogram, moving the peak towards the right in the histogram. Type 2 peaks are influenced by both the transition between the short and the intermediate populations and the intermediate and the long populations, and therefore can either be shifted to the right or to the left in the histogram. Type 3 peaks correspond to longer RR intervals in the PSP than in the histogram in most cases. Transitions between intermediate and long RR populations only decrease the inferior tail of the longest $\mathrm{RR}$ population in the conventional histogram, moving the peak towards the left in the histogram, although this has been observed in a small number of patients with three peaks detected by both methods. The existence of transition $\mathrm{RR}$ intervals between a third $\mathrm{RR}$ cluster with a hypothetic fourth RR cluster might move the position of the third peak of the RR histogram to the right, although this has not been observed in our population under study.

The proposed PSP method offers important advantages over conventional $R R$ interval histograms for the detection of multiple $R R$ interval clusters as it emphasizes most probable $\mathrm{RR}$ clusters. In this work $\mathrm{RR}$ interval histograms were compared to the PSP method in 55 patients. PSP detected all RR populations present in conventional RR interval histograms. Indeed, $67 \%$ additional RR populations were detected by the PSP method. Consequently, the PSP method may be superior to conventional RR histograms for detecting different RR clusters.

Another advantage of the PSP over existent methods is the exclusion of aberrant ventricular and ventricular ectopic beats. Suyama et. al. [56] demonstrated that pairs of RR intervals constructed with aberrant conduction 
beats were distributed along the lower envelope of the Poincaré plot. Similarly, the coupling points of wide QRS complexes are also distributed along the envelope of the Poincaré plot [103]. The PSP method excludes pairs of RR intervals that appear in the envelope of the Poincaré plot. Consequently, PSP is more robust than conventional histograms against errors in detection or classification of QRS complexes.

Improvements of the PSP against conventional RR interval histogram for the detection of RR interval clusters allow the study of shorter RR interval series which may elucidate the circadian behavior of AV nodal properties. Circadian variations and irregularity of the VR during AF have shown an important role in the prognosis of survival in patients with persistent AF [58, 104]. Preliminary works studied three-hour variations manually in the Poincaré plot by visual inspections [60]. We present an automatic method to study short-time variations on RR distributions. Different patterns of short-time variations on the VR during AF have been illustrated (Fig. 3.4). This method allows a detailed exploration of variations of each RR interval cluster along the day, however, the accuracy of short time variations on the PSP depends on the degree of organization of the VR in each patient.

The possibility to use the PS and short time variations in the PSP to monitor rate control methods is illustrated in Fig. 3.5. The reproducibility of PSP measurements in two different Holter recordings from the same patient (Fig. 3.4. panel 2 and Fig 3.5. panel 1) is confirmed. Interestingly, during rate control treatment with beta blockers, both preferential AV nodal conductions remain in similar positions. The reduction of mean RR interval was due to variations in the number of occurrences of each RR cluster rather than variations in the position of the RR clusters. In this patient beta blockade did not change the position of RR interval populations but result in shorter RR cluster less likely and less stable and longer RR cluster more probable and preferential. We illustrate the capability of this short-time monitoring technique to evaluate the effects of rate control drugs on each preferential AV node conduction.

One may take into account that a conventional $\mathrm{RR}$ interval histogram does not contain the same information as a PSP. A histogram represents the probability of occurrence of a given RR interval, whereas the PSP represents the probability of occurrence of two consecutive RR intervals with the same given value. In this work we propose the combination of the information presented in a Poincaré plot together with the information of the PSP, which is more accurate than the information of a conventional RR interval histogram. Different scenarios can be found when combining PS and PSP information (table 3.4): 1) No transition clusters are detected in the PS and only one RR cluster is detected in the PSP (18 patients in our database). These patients presented only one preferential AV nodal conduction. 2) Transition clusters are detected in the PS and more than one RR cluster is detected in the PSP (34 patients in our database). In these patients different 
RR populations were present simultaneously and the PSP allowed accurate detection of preferential AV nodal conductions.3) Multiple RR populations are detected in the PSP but transitions clusters are not detected in the PS (2 cases in our database), in this case different RR populations should be understood as RR clusters that were not present simultaneously (e.g. due to variations in the autonomic nervous system) rather than different AV node preferential conductions. 4) Transitional clusters are present in the PS but only one RR cluster is detected in the PSP ( 1 case in our database). This may happen when two preferential AV nodal conductions are present but one of them is never used two consecutive times. PSP was unable to detect the second RR cluster because there were not enough couples of consecutive RR intervals of this preferential AV nodal conduction.

Clinical implications of different AV nodal preferential conductions remain unclear. A bimodal RR histogram has been thought to indicate dual pathways AV nodal physiology during AF [40, 60, 100]. Although a bimodal distribution could be explained by the concealed conduction model in which AV node refractoriness differs between the proximal and distal region [67], Olsson et al. strongly suggested that the two different RR populations correspond to conduction through two different atrio-nodal conduction routes. In [40] HRSH were presented for the indirect characterization of multiple intranodal pathways of the AV node. However, the bimodal RR interval distribution analysis is not always related with the presence of dual AV nodal pathway physiology, and a unimodal histogram pattern may still be associated with dual AV nodal pathways [47]. In addition, the dual pathway interpretation can not explain 3 or more peaks in the PSP. Of interest is our finding that after applying PSP for the filtering of transitional RR intervals there was a high correlation between peak locations, and that the difference between peaks D3-2 and D2-1 in each patient with three peaks was almost identical. That raises the possibility that in this case the preferential AV nodal conductions represent different $\mathrm{AV}$ conduction ratios of the atrial fibrillatory input (i.e. 2:1, 3:1 and 4:1 atrioventricular conductions) and they are not necessarily determined by AV nodal anatomy. Similar atrioventricular conduction behavior takes place in more organized atrial arrhythmias such as atrial flutter. The exact properties of the atrioventricular conduction are of course hard to estimate without detailed examination during electrophysiological study, however the PSP may be a useful tool for studying the relationship between the atrial rate and preferential AV nodal conductions.

Even when the physiological meaning of the different RR populations is a controversial topic, clinical relevance of the evaluation of the existence of preferential AV nodal conductions has been reported in several studies $[60,96,97,43,99,42,100,101,54,105,106]$. Rokas et al. demonstrated that modifications in the AV node in patients presenting bimodal RR interval distributions were more effective in reducing the heart rate than in patients 
with a unimodal pattern [43]. In a more recent study from Van den Berg et al. showed that electrical cardioversion resulted more often in restoration of sinus rhythm in patients whose RR intervals were clustered, and also, those patients had a higher likelihood of remaining in sinus rhythm than their counterparts without well-defined clusters [52].

In summary, the PSP method is a robust technique that allows automatic detection of preferential AV nodal conductions. Consequently, it may play a relevant role for a deeper understanding of AF mechanisms and for quantifying $\mathrm{AF}$ patterns and properties. PSP and Poincaré plots together with already existent techniques will help in the study of rate control drugs and in the selection of a more appropriate treatment for each patient. 


\section{Chapter 4}

\section{Role of the Atrial Rate ${ }^{1}$}

\begin{tabular}{llll}
\hline 4.1 & Introduction & 4.4 & Discussion \\
4.2 & Methods & 4.5 & Conclusions \\
4.3 & Results & & \\
\hline
\end{tabular}

During atrial fibrillation (AF), RR interval histograms show different populations of predominant $R R$ intervals $(p R R)$. These $p R R$ intervals have been suggested to be multiples of the refractory period of the AV node or caused by the existence of a dual AV node physiology. In this study the hypothesis that predominant $\mathrm{RR}$ intervals are related to the dominant atrial fibrillatory rate is tested. In this study Holter ECG signals from 55 patients with persistent AF were analyzed. Number and position of predominant RR intervals were detected and compared with mean and standard deviation of the dominant atrial cycle length (DACL) . In addition, effects of an enhancement of vagal activity and rate control treatments (Beta Blockers and Verapamil) were evaluated. In all patients with more than one predominant $\mathrm{RR}$ interval and in $47 \%$ with one predominant $\mathrm{RR}$ interval, RR interval populations were statistically related with multiples of the DACL. During night activities and during beta blockers treatment, mean ventricular rate decreased $(\mathrm{p}<0.01)$. This change was associated with a variation in the percentage of occurrences of each predominant $\mathrm{RR}(\mathrm{p}<0.01)$, whereas no statistical differences were present in the mean DACL or in the position of predominant $\mathrm{RR}$ intervals. A variation of the DACL due to Verapamil was associated with a consistent modification in the position of the predominant $\mathrm{RR}$ intervals. The relation between predominant $\mathrm{RR}$ and multiples of the DACL during AF suggests that more probable RR intervals are caused by different conduction ratios of the atrial rate.

\footnotetext{
${ }^{1}$ Chapter based on the manuscript: Andreu M. Climent, M. Salud Guillem, Daniela Husser, Francisco Castells, Jose Millet and Andreas Bollmann. Role of the Atrial Rate as a Factor Modulating Ventricular Response during Atrial Fibrillation. Pacing and Clinical Electrophysiology. 33(12):1510-1517. 2010
} 


\subsection{Introduction}

Despite the large number of patients treated with rate-controlling therapies for Atrial Fibrillation (AF), atrioventricular conduction mechanisms during AF remain unclear. In fact, the ventricular response (VR) during AF presents intriguing characteristics $[53,49]$ : by constructing histograms of $\mathrm{RR}$ intervals or bidimensional histogram plots (so-called 3D plots, Lorenz plots or Poncair plots) from $R R$ series different populations of predominant $R R$ intervals ( $p R R$ ) can be identified. Whereas in some patients, unimodal RR patterns are observed, in others bi- or multimodal patterns exist. Although this phenomenon may be of importance during rate control strategies, underlying AV nodal conduction mechanisms are still an incognita. In an earlier study [107], it was postulated that $\mathrm{pRR}$ are multiples of the refractory period of the AV node. However, in more recent works pRR have been suggested to be caused by the existence of dual AV node physiology [97, 40, 43]. It was hypothesized that conduction through the slow AV nodal pathway (with a short refractory period) produces short RR intervals, whereas conductions through the fast AV nodal pathway (with a long refractory period) produces long RR intervals [97, 40, 43, 60, 41, 42, 100]. However, none of these hypotheses can explain satisfactorily the generation of the VR during AF.

The main objective of this study was the evaluation of the relationship between the positions of pRR intervals and the dominant atrial cycle length (DACL) during AF. We tested the hypothesis that the positions of $\mathrm{pRR}$ populations are caused by different conduction ratios of the atrial rate (i.e. $2 \cdot \mathrm{DACL}, 3 \cdot \mathrm{DACL}$, etc.).

\subsection{Materials and Methods}

\subsubsection{Study Population and Holter ECG Acquisition}

In this study 65 consecutive patients with persistent $\mathrm{AF}$ were included. For each patient, a clinically indicated ambulatory Holter ECGs of approximately 24 hours was recorded during usual daily activities using a CardioMem CM 3000 recorder (Fa. Getemed, Teltow, Germany). QRS complexes were detected and classified using Getemed software. Results of automatic analysis were visually inspected and each error in the detection or labeling was corrected manually. Only RR intervals between normal (sinus) beats were used in the subsequent analysis. Holter recordings with a high degree of noise or with a percentage of ventricular ectopic and aberrant beats higher than $20 \%$ were excluded $(\mathrm{N}=10)$. The remaining 55 patients comprise the study population (Table 4.1).

In order to evaluate the effect of rate control treatments, two Holter recordings were acquired for six patients (3 under beta-blockers and 3 under Verapamil). In each subgroup (beta-blockers and Verapamil), baseline 
recordings were compared to drug recordings for two patients and recordings obtained under different dosages of the same drug were compared for one patient.

Table 4.1: Patient Characteristics

\begin{tabular}{l|c|c|c|c}
\hline \hline & $\begin{array}{c}\text { Total } \\
(\mathrm{n}=55)\end{array}$ & $\begin{array}{c}\text { Unimodal } \\
(\mathrm{n}=21)\end{array}$ & $\begin{array}{c}\text { Multimodal } \\
(\mathrm{n}=34)\end{array}$ & $\mathrm{P}$ \\
\hline Age, years & $65 \pm 11$ & $67 \pm 10$ & $63 \pm 11$ & $\mathrm{NS}$ \\
Male, n (\%) & $43(78)$ & $17(81)$ & $26(76)$ & $\mathrm{NS}$ \\
AF duration, months & $19 \pm 20$ & $23 \pm 17$ & 1821 & $\mathrm{NS}$ \\
Left atrial diameter, mm & $50 \pm 8$ & $52 \pm 7$ & $50 \pm 9$ & $\mathrm{NS}$ \\
Left ventricular ejection fraction, \% & $50 \pm 14$ & $46 \pm 17$ & $53 \pm 10$ & $\mathrm{NS}$ \\
Heart disease* & $3(5)$ & $0(0)$ & $3(9)$ & $\mathrm{NS}$ \\
None, n (\%) & $23(42)$ & $7(33)$ & $16(47)$ & \\
Hypertension, n (\%) & $10(18)$ & $4(19)$ & $6(18)$ & \\
Coronary artery disease, n (\%) & $6(11)$ & $4(19)$ & $2(6)$ & \\
Valvular heart disease, n (\%) & $6(11)$ & $3(14)$ & $3(9)$ & $\mathrm{NS}$ \\
Cardiomyopathy, n (\%) & $38(69)$ & $14(66)$ & $24(71)$ & \\
Medication* & $15(27)$ & $3(14)$ & $12(35)$ & \\
Beta blockers, n (\%) & $24(44)$ & $9(42)$ & $15(44)$ & \\
Calcium channel blockers, n (\%) & $81 \pm 22$ & $79 \pm 13$ & $82 \pm 74$ & $\mathrm{NS}$ \\
Digitalis, n (\%) & & & \\
Ventricular rate, bpm & & & \\
*treatment received during recording, more than one possible, NS=not & \\
& significant & & \\
\end{tabular}

\subsubsection{Analysis of the Atrial Activity}

In order to estimate the mean atrial fibrillatory rate, the dominant frequency of atrial fibrillatory signals was estimated from the surface Holter ECG lead. Ventricular activity was reduced by subtracting a matching QRS-T template. This template was computed for each beat as a linear combination of the principal components previously obtained from the analysis of all beats in the ECG segment [108]. For every 10 seconds of ECG recording, the dominant frequency was defined as the dominant peak in the power spectrum between 3 and $10 \mathrm{~Hz}$. The dominant atrial cycle length (DACL) of each segment was calculated as the inverse of the dominant frequency. The main limitations in the estimation of the AF rate were the low signal to noise ratio of Holter signals and the possible QRS complex residue. In order to reject segments in which a precise estimation of the DACL was not possible, the spectral concentration around the dominant frequency $( \pm 0.5 \mathrm{~Hz})$ was measured. In case that this spectral concentration was lower than $40 \%$, DACL estimation was 
discarded. For each 30 minute segment, 180 values of DACL and spectral concentration were calculated ( 1 value every 10 seconds for each parameter). If more than $70 \%$ of the 10 second segments presented a spectral concentration higher than $40 \%$, mean and standard deviation of DACL of segments with high spectral concentration were computed. In addition to these parameters, mean DACL during day (7h-23h) and night (23h-7h) activity periods and during the whole day were compared.

\subsubsection{Analysis of the Ventricular Response}

The analysis of pRR during AF was based on the Poincare Surface Profile (PSP) method that has been presented in detail elsewhere [109]. In brief, this methodology allows analyzing the dynamic behavior of the VR during AF by means of the analysis of only near-equal consecutive RR intervals derived from a Poincar plot. Short-term variations in the VR were analyzed by computing a 30-minute PSP every 15 minutes, with $50 \%$ overlapping (i.e. for a Holter recording of 24 hours, 95 PSP from overlapping sequential segments can be generated), so that the number and position of pRR during the recording was monitored. For each 30 minute signal an algorithm to identify pRR on the PSP was used in order to automatically detect different RR populations [109]. For each 30 minute segment, 1, 2 or $3 \mathrm{pRR}$ intervals were detected and patients were grouped according to their VR pattern. Although the existence of two or more pRR in at least one 30-minute segment may suffice to assess the existence of a multimodal VR pattern, in order to guarantee robustness to erroneous $\mathrm{pRR}$ detections, a total percentage of PSP segments with two or more pRR greater than $10 \%$ was required for assessing multimodality.

In addition, mean ventricular rate during the day (7h-23h) and night (23h-7h) activity periods and during the whole day were compared. Moreover, position and probability of occurrence of each $\mathrm{pRR}$ were analyzed for day (7h-23h) and night (23h-7h) activity periods in all patients with more than one pRR. Specifically, for each activity period, mean position and mean percentage of RR interval occurrences around $50 \mathrm{~ms}$ of each $\mathrm{pRR}$ were measured.

\subsubsection{Comparison between Atrial Cycle Length and Predominant RR Intervals}

A pRR was considered to be an nth multiple of DACL if it was located within a window at $n \cdot\left(D A C L \pm s t d_{D A C L}\right)$. For instance, if during 30 minutes the measured DACL was $150 \pm 5 \mathrm{~ms}$, a pRR was considered a second multiple if its duration ranged from 290 to $310 \mathrm{~ms}$, a third multiple if its duration ranged from 435 to $465 \mathrm{~ms}$, etc. In order to avoid identifying relationships between DACL and pRR due to wide tolerance ranges, segments that presented a stdDACL greater than $15 \mathrm{~ms}$ were rejected. In addition, mean DACL and 
mean ventricular rate during the whole day and during day and night activity periods were compared.

\subsubsection{Statistical Analysis}

In order to evaluate the relationship between multiples of the DACL and the different RR populations, one tailed t-test was performed to evaluate the hypothesis that $p R R-n \cdot D A C L$ was lower than $n \cdot s t d_{D A C L}$ for each patient. In addition, paired Student's t tests were performed in order to compare 1) DACL between day and night, 2) ventricular rate between day and night, 3) percentage of occurrences of each pRR between day and night and 4) position of each pRR between day and night. Similar comparisons were done between baseline and rate control Holters. A p value $<0.05$ was considered statistically significant.

\subsection{Results}

\subsubsection{Atrial Cycle Length and Predominant RR Intervals}

Short-term variations of PSP and multiples of DACL of two representative patients are depicted in Figure 4.1. In the recording shown in panel 1, one peak was detected in the PSP during the whole day. The detected pRR laid inside the window of the 4th multiple of the DACL in $90 \%$ of the segments $(\mathrm{p}<0.01)$. The recording shown in panel 2 was classified as multimodal due to the existence of two pRR intervals in $25 \%$ PSP segments. In this patient the long $\mathrm{pRR}$ laid inside the window of the 5th multiple of the DACL in $100 \%$ of the 91 segments $(\mathrm{p}<0.01)$. Similarly, the short $\mathrm{pRR}$ interval appeared inside the window of the 3rd multiple of the DACL in $100 \%$ of the 23 segments $(\mathrm{p}<0.01)$.

By analyzing short time variations of PSPs over 24 hours for the whole database, 21 patients presented a unimodal VR pattern and 34 patients a multimodal VR pattern (23 with 2 pRR intervals and 11 with $3 \mathrm{pRR}$ intervals). There was no difference in clinical characteristics between patient groups (Table 4.1). Of those, 2 patients with unimodal VR patterns and 10 patients with multimodal VR patterns were excluded from further analysis due to QRST cancellation artefacts and estimation of DACL. Summarizing, 19 patients with unimodal VR pattern and 24 patients with multimodal VR pattern were analyzed.

Notice that a thorough estimation of the DACL was not possible for all 30 minute segments of these 43 patients. Thirty minute segments with an insufficient spectral concentration or with a standard deviation of the DACL larger than $15 \mathrm{~ms}$ were rejected. Patients with less than 50 segments fulfilling these criteria were not considered for further analysis. The mean number of 

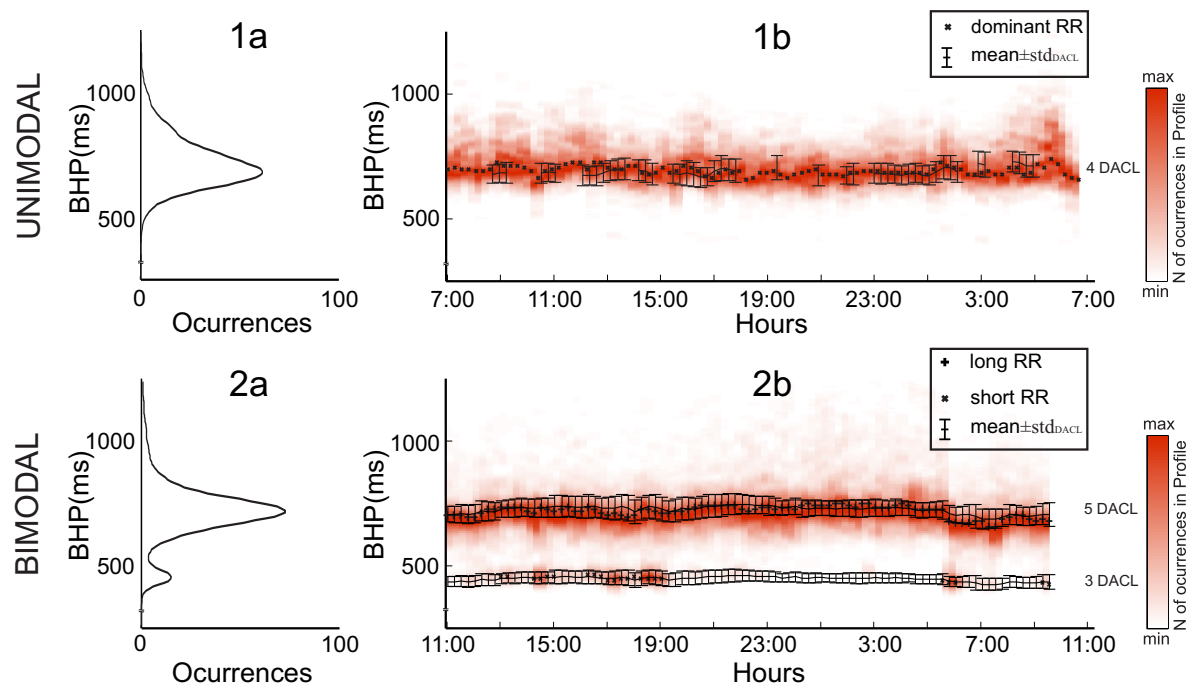

Figure 4.1: In panels 1a and 2a, 24 hours Poincaré Surface Profiles (PSP) are depicted. In panels $1 \mathrm{~b}$ and $2 \mathrm{~b}$, short-time variations of the PSP during the day are represented according to a red color scale. Crosses represent local maxima of each PSP segment automatically detected. DACL multiples are represented as the mean and standard deviation of each measurable 30-minute segment. In panels $1 \mathrm{a}$ and $1 \mathrm{~b}$, a unimodal VR is depicted in which a single pRR was found during the whole day together with the 4th multiple of DACL which was statistically related with the pRR. In panels $2 \mathrm{a}$ and $2 \mathrm{~b}$, a multimodal patient is depicted in which a bimodal PSP was detected during several segments of the day, the 3rd and 5th multiples of DACL were statistically related with pRR intervals. 
measurable segments and the percentage of them that satisfy the relation between atrial cycle length and pRR interval are shown in table 4.2.

For all patients with a multimodal VR pattern and for 9 of the 19 patients with a unimodal VR pattern it was statistically demonstrated that $\mathrm{pRR}$ intervals were harmonics of the DACL. For these 9 patients in $75 \pm 15 \%$ of measurable segments $\mathrm{pRR}$ was statistically related to DACL $(\mathrm{p}<0.05)$. In the other 10 patients with a unimodal VR pattern, $56 \pm 21 \%$ of segments presented a pRR$\mathrm{n} \cdot \mathrm{DACL}$ lower than $s t d_{D A C L}(\mathrm{p}=\mathrm{ns})$ Notice that the standard deviation of the position of the pRR interval in these 10 patients was $90 \pm 50 \mathrm{~ms}$, significantly higher than in the other 9 patients with a unimodal pattern $(50 \pm 23 \mathrm{~ms}, \mathrm{p}<0.05)$. These patients may have been incorrectly classified into the unimodal group and present two nearby $\mathrm{pRR}$ populations leading to an inaccurate location of the pRR at some segments and thus inability to demonstrate its relation with the DACL.

Table 4.2: Measurable segments and percentage of them statistically related with atrial cycle length.

\begin{tabular}{|c|c|c|c|}
\hline & $\mathrm{N}$ & $\begin{array}{l}\text { Measured } \\
\text { Segments }\end{array}$ & $\begin{array}{c}\mathrm{pRR}-\mathrm{n} \cdot \mathrm{DACL} \\
< \\
\mathrm{n} \cdot \mathrm{std}_{D A C L}\end{array}$ \\
\hline UNIMODAL PSP & 19 & $76 \pm 43$ & $65 \pm 22 \%$ \\
\hline MULTIMODAL PSP & 24 & $79 \pm 36$ & $85 \pm 11 \%$ \\
\hline
\end{tabular}

\subsubsection{Autonomic Tone Effects}

Comparison of day and night periods for all patients with more than one pRR is summarized in table 4.3. No statistical differences were found neither in the mean DACL nor in the position of each pRR interval, although the mean ventricular rate was significantly lower for night periods. This decrease was associated with an increase in the proportion of long $\mathrm{pRR}$ in comparison to short $\mathrm{pRR}$. The number of patients with a third $\mathrm{pRR}$ was too small to detect statistically significant trends. An example of the stability of the position of $\mathrm{pRR}$ and their day-to-night variation of occurrences is illustrated in panel $2 \mathrm{~b}$ of Figure 4.1. In this patient, during the night, number of occurrences of the pRR corresponding to the 3rd atrial harmonic decreased markedly whereas the number of occurrences of the pRR corresponding to the 5th atrial harmonic increased.

For the 9 patients that presented unimodal ventricular response pattern and pRR was demonstrated to be a multiple of the DACL, mean RR interval were longer during night periods $(767 \pm 181 \mathrm{~ms}$ vs. $849 \pm 187 \mathrm{~ms}, \mathrm{p}<0.05)$. However, 
Table 4.3: Comparison between Day and Night Activity Periods.

\begin{tabular}{|c|c|c|c|c|c|c|c|c|}
\hline & & \multicolumn{3}{|c|}{ Day (7h-23h) } & \multicolumn{3}{|c|}{ Night (23h-7h) } & \multirow[b]{2}{*}{ ** } \\
\hline RR mean & $\mathrm{ms}$ & 711 & \pm & 131 & 832 & \pm & 158 & \\
\hline DACL mean & $\mathrm{ms}$ & 160 & \pm & 20 & 161 & \pm & 21 & \\
\hline \multirow[t]{2}{*}{$1 \mathrm{pRR}$} & $\mathrm{ms}$ & 505 & \pm & 142 & 489 & \pm & 166 & \\
\hline & $\%$ oc. & 29 & \pm & $14 \%$ & 14 & \pm & $12 \%$ & $* *$ \\
\hline \multirow[t]{2}{*}{$2 \mathrm{pRR}$} & $\mathrm{ms}$ & 782 & \pm & 162 & 792 & \pm & 170 & \\
\hline & $\%$ oc. & 24 & \pm & $15 \%$ & 30 & \pm & $13 \%$ & $* *$ \\
\hline \multirow[t]{2}{*}{$3 \mathrm{pRR}$} & $\mathrm{ms}$ & 1116 & \pm & 251 & 1123 & \pm & 257 & \\
\hline & $\%$ oc. & 11 & \pm & $13 \%$ & 12 & \pm & $5 \%$ & \\
\hline
\end{tabular}

$\mathrm{DACL}=$ dominant atrial cycle length, $\mathrm{pRR}=$ predominant $\mathrm{RR}$ interval, $\mathrm{oc}=$ occurrences.

two different mechanisms produce these reductions in the ventricular rate. In six patients, a significant variation of the mean DACL was detected between day and night $(157 \pm 14 \mathrm{~ms}$ vs $165 \pm 16 \mathrm{~ms}, \mathrm{p}<0.05)$. In these patients both day and night periods presented a pRR similar to the 5th multiple of the DACL and so the position of the pRR changed following the mean DACL variations. In three patients, no significant differences were measurable for the mean of the DACL between day and night. However, in these three patients, pRR was the 4th multiple of the mean DACL during the day whereas during the night pRR was the 5 th multiple of the mean DACL.

\subsubsection{Rate Control Effects}

Comparisons between Holter recordings during baseline and rate control treatment for the three patients that were treated with Betablockers and for the three patients treated with Verapamil are summarized in table 4.4.

On one hand, patients treated with Betablockers presented a multimodal ventricular response pattern with 2 populations during both recordings. No significant differences were found neither in the mean atrial rate nor in the position of predominant $\mathrm{RR}$ intervals, however mean ventricular rate was significantly lower $(\mathrm{p}<0.01)$. This reduction in the ventricular rate was associated with an increase in the percentage of occurrences of long pRR compared to short pRR $(\mathrm{p}<0.01)$. In Figure 4.2 the effects of Betablockers in the VR pattern and its relation with the atrial rate in patient 1 are illustrated. As visible, in both Holter recordings positions of $p R R$ were similar $(p=n . s$. and in both the positions of these populations were significantly related with 3rd and 5 th harmonics of the atrial rate $(\mathrm{p}<0.05)$.

On the other hand, patients treated with Verapamil presented a multimodal ventricular response pattern during both recordings. In these patients a significant reduction in the ventricular rate was also achieved. 

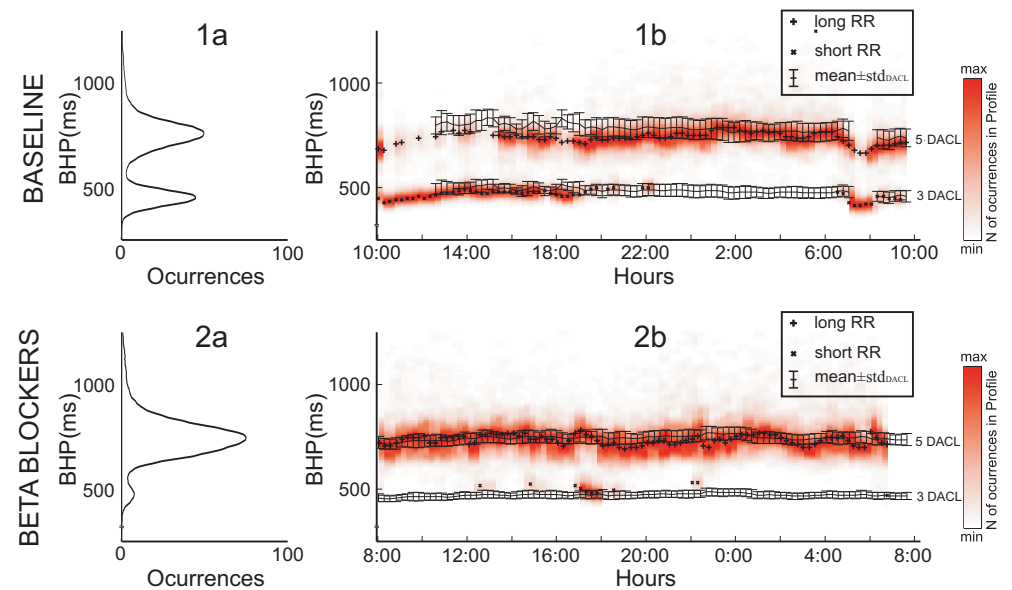

Figure 4.2: Panels 1a and 1b represent 24 hours PSP and short-time variations of the PSP together with the $3 \mathrm{rd}$ and 5 th atrial rate multiples (panels $1 \mathrm{~b}$ and $2 \mathrm{~b}$ ) during a baseline Holter (panels 1a and 1b) and during rate control treatment with beta blockers (panels $2 \mathrm{a}$ and $2 \mathrm{~b}$ ).

However, under Verapamil treatment a significant reduction in the DACL was measured during the second Holter recordings $(\mathrm{p}<0.01)$. Positions of $\mathrm{pRR}$ in the second Holter were also shifted, but still remained statistically related with harmonics of the DACL. Nevertheless, although DACL decreased, positions of the pRR increased due to an increase in the harmonic order (table 4.4).

\subsection{Discussion}

\subsubsection{Main Findings}

In this study, a relation between the atrial cycle length and the ventricular response during AF has been found. For the first time during AF, the position of predominant RR intervals has been shown to be correlated with multiples of the dominant atrial cycle length. It has been illustrated how the reduction of the ventricular rate due to changes in the autonomic tone (e.g. days vs. night) does not imply variations in the position of predominant RR intervals, but rather variation in the probability of occurrence of each predominant RR intervals. Similar behavior was found when the conduction properties of the AV node were modified by Betablockers. In addition, it has been illustrated how a modification in the atrial rate due to Verapamil produce shifts in the position of the pRR consistent with modified DACL values. 
Table 4.4: Rate Control Treatment Effects.

\begin{tabular}{c|c|c|c|c|c|c|c|c}
\hline \hline \multicolumn{1}{c}{ Baseline Recordings } \\
\hline $\mathrm{N}$ & RR & DACL & \multicolumn{3}{|c|}{ pRR } & \multicolumn{3}{c}{2 pRR } \\
& mean & mean & $(\mathrm{ms})$ & harm. & $\%$ oc. & $(\mathrm{ms})$ & harm. & $\%$ oc. \\
\hline 1 & $735 \pm 220$ & $151 \pm 11$ & $458 \pm 24$ & 3rd & $24 \%$ & $760 \pm 30$ & 5 th & $39 \%$ \\
2 & $914 \pm 233$ & $171 \pm 13$ & $703 \pm 60$ & 4 th & $24 \%$ & $1018 \pm 39$ & 6 th & $14 \%$ \\
3 & $600 \pm 195$ & $170 \pm 11$ & $502 \pm 35$ & 3rd & $39 \%$ & $849 \pm 29$ & 5 th & $22 \%$ \\
\hline
\end{tabular}

\begin{tabular}{c|c|c|c|c|c|c|c|c}
\hline \hline \multicolumn{10}{c}{ Beta Blockers Recordings } \\
\hline $\mathrm{N}$ & RR & DACL & \multicolumn{3}{c|}{$1 \mathrm{pRR}$} & \multicolumn{3}{c}{$2 \mathrm{pRR}$} \\
& mean & mean & $(\mathrm{ms})$ & harm. & $\%$ oc, & $(\mathrm{ms})$ & harm. & $\%$ oc, \\
\hline 1 & $788 \pm 205$ & $154 \pm 12$ & $464 \pm 23$ & $3 \mathrm{rd}$ & $4 \%$ & $759 \pm 23$ & 5 th & $49 \%$ \\
2 & $996 \pm 221$ & $171 \pm 14$ & $706 \pm 53$ & 4 th & $19 \%$ & $1018 \pm 27$ & 6 th & $22 \%$ \\
3 & $683 \pm 187$ & $168 \pm 16$ & $497 \pm 31$ & $3 \mathrm{rd}$ & $29 \%$ & $839 \pm 36$ & 5 th & $36 \%$ \\
\hline
\end{tabular}

\begin{tabular}{c|c|c|c|c|c|c|c|c}
\hline \hline \multicolumn{10}{c}{ Baseline Recordings } \\
\hline $\mathrm{N}$ & RR & DACL & \multicolumn{2}{c}{ 1 pRR } & \multicolumn{2}{c}{2 pRR } & \multicolumn{2}{|c}{3 pRR (ms) } \\
& mean & mean & $(\mathrm{ms})$ & harm. & $(\mathrm{ms})$ & harm. & $(\mathrm{ms})$ & harm. \\
\hline 4 & $773 \pm 242$ & $178 \pm 16$ & $367 \pm 11$ & 2nd & $539 \pm 21$ & 3rd & $711 \pm 21$ & 4 th \\
5 & $911 \pm 225$ & $146 \pm 14$ & $720 \pm 39$ & 5th & $1017 \pm 30$ & 7 th & & \\
6 & $752 \pm 162$ & $171 \pm 13$ & $504 \pm 25$ & 3rd & $672 \pm 43$ & 4 th & & \\
\hline
\end{tabular}

\begin{tabular}{c|c|c|c|c|c|c|c|c}
\hline \hline \multicolumn{10}{c}{ Verapamil Recordings } \\
\hline $\mathrm{N}$ & RR & DACL & \multicolumn{2}{c}{1 pRR } & \multicolumn{2}{c}{2 pRR } & \multicolumn{2}{|c}{3 pRR (ms) } \\
& mean & mean & $(\mathrm{ms})$ & harm. & $(\mathrm{ms})$ & harm. & $(\mathrm{ms})$ & harm. \\
\hline 4 & $870 \pm 199$ & $153 \pm 14$ & $625 \pm 21$ & 4 th & $781 \pm 28$ & 5 th & $910 \pm 30$ & 6 th \\
5 & $942 \pm 233$ & $134 \pm 13$ & $668 \pm 32$ & 5 th & $931 \pm 38$ & 7 th & $1208 \pm 38$ & 9 th \\
6 & $873 \pm 196$ & $157 \pm 14$ & $631 \pm 31$ & 4 th & $797 \pm 39$ & 5 th & & \\
\hline
\end{tabular}

$\mathrm{N}=$ patient number, $\mathrm{DACL}=$ dominant atrial cycle length, $\mathrm{pRR}=$ predominant $\mathrm{RR}$ interval, harm.: harmonic order respect to mean DACL. Mean values in ms.

\subsubsection{Ventricular Response during Atrial Fibrillation}

The VR during AF is usually assumed to be a random phenomenon [110]. However, the existence of a certain relationship between each RR interval and the preceding RR series was demonstrated by means of bidimensional histograms and autocorrelation functions [111, 52], where different populations of more probable RR intervals ( $\mathrm{pRR}$ ) can be found. Despite the importance of these phenomena in rate-controlling therapies for the AF, mechanisms responsible of this observation remain controversial. In 1950, Sderstrm postulated that $\mathrm{pRR}$ conductions should be multiples of the refractory periods of the AV node [107]. Although it was a reasonable hypothesis, it does not explain satisfactorily the variations of $\mathrm{pRR}$ during day and night or 
Betablockers. Since AV nodal refractory period is modified by the vagal tone [112], position of pRR should gradually change between day and night, following the vagal tone. Rather than this, the reduction of the mean RR during the night was caused by an increase in the number of occurrences of long RR interval populations and a decrease in the number of occurrences of short RR interval populations, whereas the position of populations did not change. A similar behavior was also observed in the three patients treated with Betablockers.

In 1956, Moe et al. presented evidence of the existence of an AV node dual physiology [23]. It was demonstrated that the so-called slow-pathway has a shorter refractory period but lower conduction velocity than the so-called fast-pathway. The concept of two pathways with separated conduction properties was used later by Olsson et al. [97, 40, 95] for suggesting that pRR intervals during $\mathrm{AF}$ are caused by conduction through different AV node pathways. However, even when the slow pathway presents a slightly shorter refractory period than the fast pathway, both refractory periods range from 250 to $350 \mathrm{~ms}$ [113], differences that do not exceed $100 \mathrm{~ms}$, far from the positions of the pRR intervals observed in our results and previous studies [40, 41]. According to our results, short and long RR distributions, or even multiple RR populations, do not necessarily imply conduction though different AV nodal pathways [40]. In fact, Garrigue et al. demonstrated that not the dual AV nodal pathways per se, but the complexity of the atrial-AV nodal engagement underlie the VR patterns observed during high atrial rates $[114,115]$. In the same way, by pacing from different sites of the atria in rabbit experiments, Chorro et al. [116] concluded that the ventricular rate during AF was not only determined by the properties of the AV node but also by the rate and irregularity of the fibrillatory process. This reaffirms the idea presented by Kirsh et al.[117], who suggested that the irregularity of the VR to AF is primarily a consequence of the irregularity inherent in the atrial activity and the role of the AV node is predominantly confined to that of scaling the atrial activity [117]. Our findings relating pRR and the mean atrial cycle length in Holter ECG recordings from humans are consistent with these findings.

\subsubsection{Atrioventricular Conduction Mechanisms}

According to our results, the role of the AV node may be mainly confined to scaling atrial waves and, consequently, in order to study the VR during AF a careful evaluation of the rate and organization degree of the atrial fibrillatory activity bombarding the AV node is necessary. Our results suggest that atrioventricular conduction during AF is based on similar mechanisms to that of regular atrial tachycardias such as Atrial Flutter. This novel point of view should modify the way in which rate control strategies aim at achieving regular and optimal ventricular rates. Nevertheless, the atrioventricular conduction during AF is the result of a combination of several effects. Although more 
probable RR intervals have been related to the atrial rate, the way in which concealed beats, interactions between AV nodal pathways or the autonomic nervous system modulate the conduction of atrial waves needs to be clarified.

\subsubsection{Limitations}

In this manuscript some of the most novel techniques have been used in order to evaluate globally the atrial activity and the ventricular response during AF $[108,109]$. These techniques have been applied to Holter ECG recordings since only long recordings allow a detailed analysis of autonomic tone or rate control effects in the atrial and ventricular response during AF. Although the evaluation of global phenomenon rather than detailed analysis of the AV node zone could implicate some limitations, the assumption that DACL could be calculated from ECG signals was based on the demonstrated stable relation between rate of ECG atrial waves and endocardial cycle length [118]. Notice that only segments with an accurate estimation of the DACL were used for the comparison. In addition, the assumption that VR during AF could be characterized by means of BHP was based on the demonstrated relation between position of pRR in BHPs and in complete histograms [109]. Although these assumptions have been accepted to obtain a first approximation of the overall relation between atrial activity and the VR during AF, more complex models including atrial activity organization parameters and the full population of ventricular activations would help in a further understanding of the beat to beat dynamics of the AV node during AF. In fact, further analysis evaluating nervous stimulations [119] and atrial wavefront organization should be needed in order to confirm our hypothesis and to reveal the reason why some patients presented unimodal or multimodal VR patterns.

\subsection{Conclusions}

The relation between predominant RR and multiples of the DACL suggests that more probable $\mathrm{RR}$ intervals are caused by different conduction ratios of the atrial rate. This finding represents a novel hypothesis and may contribute to a better understanding of the role of the AV node during AF. 


\section{Chapter 5}

\section{Generation of Realistic AA Interval Series ${ }^{1}$}

\subsection{Introduction \\ 5.2 Materials and Methods 5.3 Results}

\author{
5.4 Discussion and \\ Conclusions
}

The aim of the this chapter is to describe a methodological architecture for the generation of realistic atrial to atrial activation intervals (AA) during atrial fibrillation $(\mathrm{AF})$, which can be used to investigate the role of the atrial fibrillatory process in the ventricular response during AF. In this work, a methodology for the generation of AA intervals series with selected values for mean, standard deviation, kurtosis, skewness and with specific autocorrelation functions is presented and evaluated. In order to test the accuracy of this method, AA times were calculated from two endocardial recordings of 10 patients during AF. In addition, the role of each statistical characteristic of the AA interval series in the ventricular response during AF is examined using a mathematical model of the $\mathrm{AV}$ together with realistic atrial activation sequences generated by using the presented methodology. We have observed RR histograms with multiple RR populations occurring at multiples of the mean atrial rate, as observed in real AF recordings. In addition, we have shown that the statistical characteristics of the AA series influence the shape of the RR histogram, demonstrating the importance of an accurate characterization and generation of AA interval series during AF. The use of the present methodology may help in understanding the role of the atrial rate in the ventricular response during $\mathrm{AF}$.

\footnotetext{
${ }^{1}$ Chapter based on the manuscript: Andreu M. Climent, Felipe Atienza, Jose Millet and M. Salud Guillem. Generation of Realistic Atrial to Atrial Interval Series during Atrial Fibrillation. Submitted
} 


\subsection{Introduction}

Atrial fibrillation (AF) is characterized by disorganized electrical activity of the atria. This is associated with an irregular bombarding of the atrial activity into the AV node $[4,3]$. Since the AV node is not able to conduct all atrial activations, some of them are blocked within the node. This "filtering" property of the AV node is fundamental for keeping the heart beating at a sustainable rate. However, the ventricular response during AF presents shorter and more irregular RR intervals than during sinus rhythm. This fast, irregular ventricular rate associated with $\mathrm{AF}$ can cause some of the most severe symptoms such as palpitation, chest discomfort, light-headiness and syncope $[4,3]$. In fact, rapid and irregular heart rate may result in the heart being unable to provide adequate blood flow and oxygen delivery to the rest of the body with all the associated long-term problems. The so-called rate control strategy of AF focuses on controlling ventricular rate. Usually, this treatment strategy deals with efforts to adjust propagation properties of the AV without taking into account the atrial fibrillatory process [3]. Nevertheless, the important role of the atrial fibrillatory process in the ventricular response pattern during $\mathrm{AF}$ has recently been proved [116, 120, 121, 115, 114]. This research demonstrated that in order to understand the atrioventricular conduction during AF and thus achieve an optimal rate control it is necessary to combine an accurate characterization of the atrial to atrial activation interval (AA interval) with a model of the AV conduction.

In the literature great efforts have been made in order to characterize and model the role of the atrioventricular junction in the ventricular response during $\mathrm{AF}$ (i.e. RR interval series) [67, 64, 65, 72, 74, 73, 70]. However, little attention has been paid to the specific characteristics of AA interval series and its relation to the ventricular response. Often, AA interval series during AF have been considered as a completely random phenomenon presenting either a Gaussian or a Poisson distribution [67, 94, 122, 123, 90, 71] and these random series were used as inputs for mathematical models of the atrioventricular conduction during $\mathrm{AF}[67,74,71]$. These approximations allow the modification of the mean AA interval and its standard deviation and thus are appropriate for testing the role of some conduction properties of the AV node junction such as the effective refractory period or the conduction velocity in the ventricular response. However, the role of some important characteristics of the atrial activation pattern cannot be evaluated. Specifically, the peakedness and the degree of asymmetry of a distribution of AA intervals may modify the ventricular response pattern. And more importantly, the possible presence of certain temporal organization in atrial wavelets, which indicates that atrial activation during $\mathrm{AF}$ is not entirely random [124, 72, 125] may influence the variability of $\mathrm{RR}$ interval series. In this study, we describe a methodological architecture for the generation of realistic AA intervals following a suitable PDF and with a desired autocorrelation function. 
Specifically, we have chosen the Type IV Pearson distribution which allows for varying degrees of skewness and kurtosis independently of mean and standard deviation moments $[126,127,128,129,130,131]$ and thus it is well suited for modeling many biological applications where distributions of interest are often asymmetric and have extensive tails.

This chapter is organized as follows: first, the methodology for the generation of AA intervals with selected values for mean, standard deviation, kurtosis, skewness and with specific autocorrelation functions is presented. The accuracy of the presented methodology to generate realistic AA interval series is tested and compared to other distributions used in the literature. Finally, the role of each statistical characteristic of the AA interval series in the ventricular response during $\mathrm{AF}$ is illustrated by using a mathematical model of the AV together with realistic atrial activation sequences generated by using the presented methodology.

\subsection{Methods}

\subsubsection{Methodology for Generating Atrial to Atrial Interval Series}

Realistic AA intervals can be synthesized by first generating a random series with a desired PDF and then forcing the series to present a given autocorrelation function. Both the PDF of choice and the autocorrelation introduced should emulate the process of arrival of atrial impulses to the AV node. In the literature, Poisson and Gaussian distributions have been used to model the PDF of AA intervals during AF. In a Poisson distribution all its statistical moments are determined by a single parameter ( $\lambda$ and thus statistical characteristics of the distribution (i.e. mean, standard deviation, skewness and kurtosis) cannot be set independently. In the case of a Gaussian distribution, the mean and the standard deviation can be set independently, but the kurtosis and the skewness have constant values, (i.e. 3 and 0 respectively).

The Type IV Pearson distribution allows for varying degrees of mean, standard deviation, skewness and kurtosis, and so is ideally suited for many applications in which distributions of interest are asymmetrical and have extensive tails. This is the case of several biological phenomena and in particular of the AA interval series during AF. The expression of Type IV Pearson distribution is given by $[126,127,128,129,130,131]$ :

$$
f(x)=C \cdot\left[1+\left(\frac{x-\mu_{4}}{\mu_{3}}\right)^{2}\right]^{-\mu_{1}} \cdot \exp \left[-\mu_{2} \cdot \arctan \left(\frac{x-\mu_{4}}{\mu_{3}}\right)\right]
$$

where $\mu_{1}$ and $\mu_{2}$ are shape parameters which jointly determine the degree of skewness and kurtosis of the distribution, and $\mu_{3}$ and $\mu_{4}$ are scale and location 
parameters. These four parameters characterize the distribution and uniquely determine the first four moments of the distribution (mean, variance, skewness and kurtosis) [127, 130]:

$$
\begin{gathered}
\mu_{1}=(r+2) / 2 \\
\mu_{2}=\frac{-r(r-2) S_{k}}{\sqrt{16(r-1)-S_{k}^{2}(r-2)^{2}}} \\
\mu_{3}=\sqrt{V\left[r-1-S_{k}^{2}(r-2)^{2} / 16\right]} \\
\mu_{4}=M-(r-2) S_{k} \sqrt{(V) / 4}
\end{gathered}
$$

where

$$
r=\frac{6\left(K-S_{k}^{2}-1\right)}{2 K-3 S_{K}^{2}-6}
$$

and $M, V, S_{k}$ and $K$ are respectively the mean, variance, skewness and kurtosis of the distribution. The normalization parameter $C$ depends on $\mu_{1}, \mu_{2}$ and $\mu_{3}[126,128,129,131]$ :

$$
C=\left[\mu_{3} \int_{p i / 2}^{-p i / 2} \cos ^{r}(x) \exp \left(-\mu_{2} x\right) d x\right]^{-1}
$$

which can be computed via numerical integration [127]. Note that Type IV Pearson distribution requires the following condition to be satisfied [128]:

$$
0<k=\frac{{S_{k}}^{2}(K+3)^{2}}{4\left(4 K-3{S_{k}}^{2}\right)\left(2 K-3{S_{k}}^{2}-6\right)}<1
$$

Once a closed-form of the desired distribution is chosen and its statistical moments are set to resemble realistic AA series, random series following this distribution can be computed from uniform random series, as described by Devroye [132] and Nagahara [133]. This process is illustrated in Fig. 5.1. The computed AA series is thus a sequence of uncorrelated AA intervals with the desired PDF. Correlation among AA intervals can be introduced by filtering the uncorrelated AA series $\left(A A_{u}\right)$ with the desired autocorrelation coefficients [122]:

$$
A A_{c}[n]=\sum_{k=0}^{M} c_{k} A A_{u}[n-k]
$$

where $c$ is the desired autocorrelation coefficients and $A A_{c}$ is the generated AA interval series. 


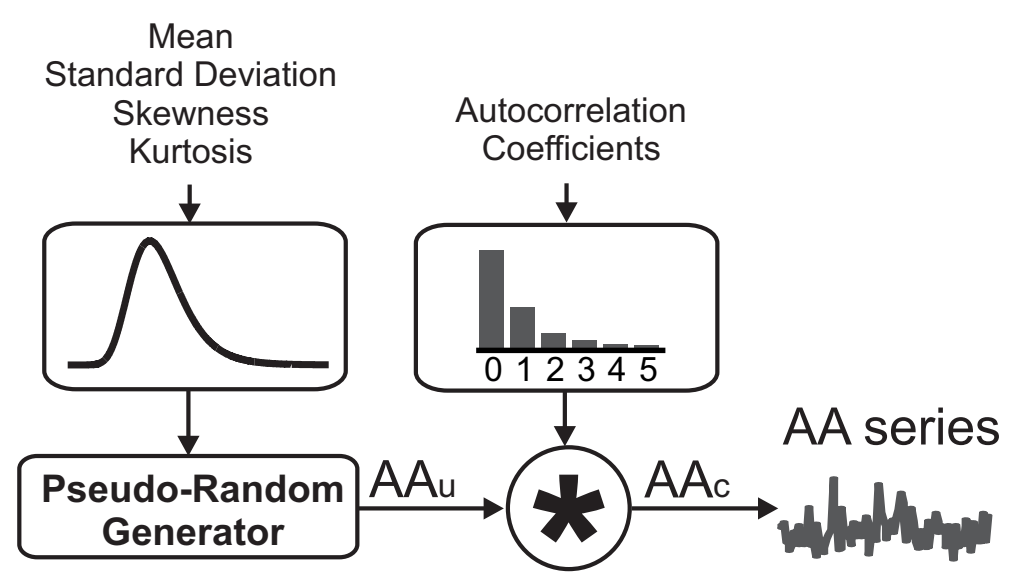

Figure 5.1: Schematic representation of the procedure used to generate realistic AA interval series during Atrial Fibrillation. Type IV Pearson Distribution (characterized by mean, standard deviation, skewness and kurtosis) is used to generate a pseudo-random uncorrelated AA series $\left(A A_{u}\right)$. The output of the generator is convoluted with a given autocorrelation function in order to obtain the desired $A A_{c}$ interval series.

\subsubsection{Comparison with real AA interval series}

In order to demonstrate the accuracy of the presented methodology in generating realistic AA interval series, endocardial electrograms recorded during AF were used. Statistical characteristics of real recordings $\left(A A_{r}\right)$ were used as input parameters for the generation of synthetic AA interval series $\left(A A_{c}\right)$ and finally, similarities between $A A_{r}$ and $A A_{c}$ were evaluated.

Specifically, bipolar endocardial electrograms were obtained in 10 patients during AF prior to radiofrequency ablation for the termination of the arrhythmia 1. For each patient, endocardial recordings from the right and left atrium were obtained. Atrial activations were automatically detected from the recordings by using the methodology presented by Faes et al. [134]. Briefly, after electrogram filtering, threshold levels were set to detect atrial activations. A total of $20 \mathrm{AA}$ interval series were used to test the presented AA generator. In order to develop the analysis independently from the mean AA interval, only the first 2000 AA intervals of each recording were used.

Accuracy of Type IV Pearson distribution in modeling the PDF of real AA interval series was measured by means of the Kullback-Leibler distance [135] and compared with that of Gaussian and Poisson distributions.

In order to estimate the ability of the presented methodology to generate realistic AA interval series, statistical parameters of recorded AA intervals $\left(A A_{r}\right)$ were used as inputs for constructing synthetic $A A_{c}$ series. Differences 
between statistical measurements extracted from the generated AA series $\left(A A_{c}\right)$ and those extracted from real AA interval series $\left(A A_{r}\right)$ were compared by linear regression. Also, a paired, two-tailed Student's t-test (pi0.05) was used to evaluate the dissimilarity of each measurement pair. SPSS 16.0 (SPSS Inc., Chicago, Illinois) was used for the analysis. In addition, standard and bidimensional histograms (so-called Lorenz or Poincaré plots [109]) of real and generated AA interval series (i.e. $A A_{r}$ and $A A_{c}$ ) were displayed and visually compared.

\subsubsection{Role of Statistical Characteristics of the Atrial to Atrial Activity in the Ventricular Response during AF}

Finally, the influence of each statistical characteristic of the AA interval series on the ventricular response during $\mathrm{AF}$ was illustrated by using a mathematical model of the AV node together with the realistic atrial activation sequences generated by using the presented methodology. Specifically, the mathematical model of human AV nodes proposed by Mangin et al. was used [73]. This mathematical model estimates the atrioventricular conduction time of a specific atrial beat $\left(A_{i+1}\right)$ by means of the so-called AV nodal recovery curve which was approximated by an exponential function:

$$
A V_{i+1}=A V_{\min }+\alpha \cdot \exp \left(-\frac{A_{i} A_{i+1}-A V_{i}}{\tau}\right)
$$

where $A V_{i+1}$ is the conduction time for the atrial beat $A_{i+1}, A V_{\min }$ is the minimum conduction time corresponding to the propagation in full recovery tissue, $\alpha$ and $\tau$ are positive constants, $A_{i} A_{i+1}$ is the atrial to atrial interval measured from the last conducted beat Ai to the testing beat $A_{i+1}$ and $A V_{i}$ is the atrioventricular conduction time of the last conducted beat. In this model an atrial beat is considered to be blocked if it reaches the AV node during its refractory period. This refractory period is set to a constant value $\theta$ which is incremented by a random quantity $\Delta$. In this study, we have set the parameters of the model to the midpoint of their physiological values $[72,73,70]\left(A V_{\min }=50 \mathrm{~ms}, \alpha=200 \mathrm{~ms}, \tau=100 \mathrm{~ms}, \theta=200 \mathrm{~ms}\right.$ and $\Delta$ a random number with mean $50 \mathrm{~ms}$ and standard deviation $50 \mathrm{~ms}$ ).

In order to evaluate the separate effect of each statistical property of the AA interval series on the ventricular response, we generated sets of AA series in which one parameter (mean, standard deviation, skewness, kurtosis or autocorrelation) was variable in a physiologically feasible range and the remaining parameters were set to their most common value, as observed in human patients. 


\subsection{Results}

\subsubsection{Accuracy of Pearson Type IV distribution}

In Fig. 5.2, a fit of Poisson, Gaussian and Pearson Type IV distributions to a real AA series during AF is illustrated. Both Gaussian and Poisson distributions were less accurate than the Pearson Type IV PDF. Measurement of the Kullback-Leibler distance in all evaluated AA series confirms this observation, as summarized in Table 5.1. The distances between fitted Type IV Pearson distributions and real data were significantly shorter than those measured for Gaussian or Poisson distributions $(\mathrm{p}<0.001)$. Notice that the condition for Type IV Pearson Distribution adjustment in eq. 5.8 was always satisfied.

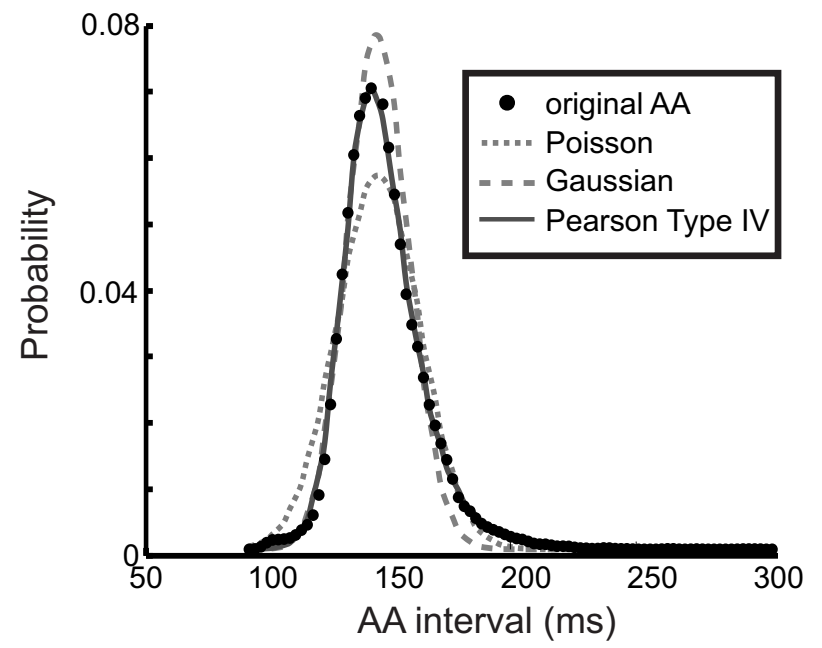

Figure 5.2: Probability distribution of a real AA interval series and a fitted synthetic function. Poisson, Gaussian and Pearson Type IV distributions are plotted.

Table 5.1: Kullback- Leibler Distances for all Distributions

\begin{tabular}{cc}
\hline \hline Distribution & Kullback-Leibler distance \\
\hline Poisson & $0.641 \pm 0.440$ \\
Gaussian & $0.105 \pm 0.066$ \\
Pearson Type IV & $0.025 \pm 0.011$ \\
\hline
\end{tabular}




\subsubsection{Accuracy of Generated AA interval series}

In Fig. 5.3, an example of a real AA interval series $\left(A A_{r}\right)$ recorded in the left atrium and the $A A_{c}$ series generated using its statistical parameters are depicted. As it can be noted, although the specific AA interval values are different, similar AA interval histograms (panels B), bidimensional histograms (panels C) and autocorrelation coefficients (panels D) were obtained.

In Fig. 5.4 the analysis of the whole database is shown. Statistical parameters of recorded $A A_{r}$ interval series and their corresponding synthetic $A A_{c}$ interval series for all recordings are depicted (i.e. mean, standard deviation, skewness, kurtosis, 1st and 2nd order autocorrelation coefficients). There was a significant correlation between all real and generated statistical parameters $\left(R^{2}>0.92, \mathrm{p}<0.01\right)$.

Although all recordings were acquired during AF, there was a wide range of variation of each parameter, which demonstrates that statistical parameters of AA series are not homogeneous. Skewness was higher than zero in all analyzed patients and ranged from 0.06 to $2.24(\mathrm{p}<0.01)$, and kurtosis was higher than 3 in all analyzed recordings and ranged from 3.28 to $13.27(\mathrm{p}<0.01)$.

The results of autocorrelation analysis are illustrated in Fig. 5.4. Although autocorrelation coefficients up to the fifth order were used for the generation of AA interval series, none of the analyzed recordings presented 3rd to 5th order coefficients higher than 0.2 . For this reason, only 1 st and 2 nd order autocorrelation coefficients are illustrated. $A A_{r}$ series were not uncorrelated, since in $40 \%$ of the analyzed recordings AA intervals presented a significant dependency with the previous AA interval (displaying a 1st order autocorrelation coefficient higher than 0.2). Again, measured autocorrelation coefficients were not homogeneous in our database which reflects that different degrees of temporal organization exist in AF patients. Specifically, the range for 1 st and 2nd autocorrelation coefficients was -0.03 to 0.46 and -0.02 to 0.28 respectively.

\subsubsection{Role of Statistical Characteristics of the AA series in the Ventricular Response during AF}

In Fig. 5.5, histograms corresponding to different sets of AA series are illustrated in which only one statistical parameter of the series was varied. Notice that two RR populations appear in RR histograms which correspond to multiples of the mean atrial rate while the overall shape of the histogram depends on several statistical characteristics of the AA series. The mean value of the AA series has a great impact on the shape of the RR histogram, as panel A shows, modifying both the location of the dominant populations and the number of occurrences of each population. For fast atrial rates, higher multiples of the mean AA occur with a higher probability (i.e. 2nd and 3rd multiples), whereas for slower atrial rates lower multiples of the mean AA present more occurrences 

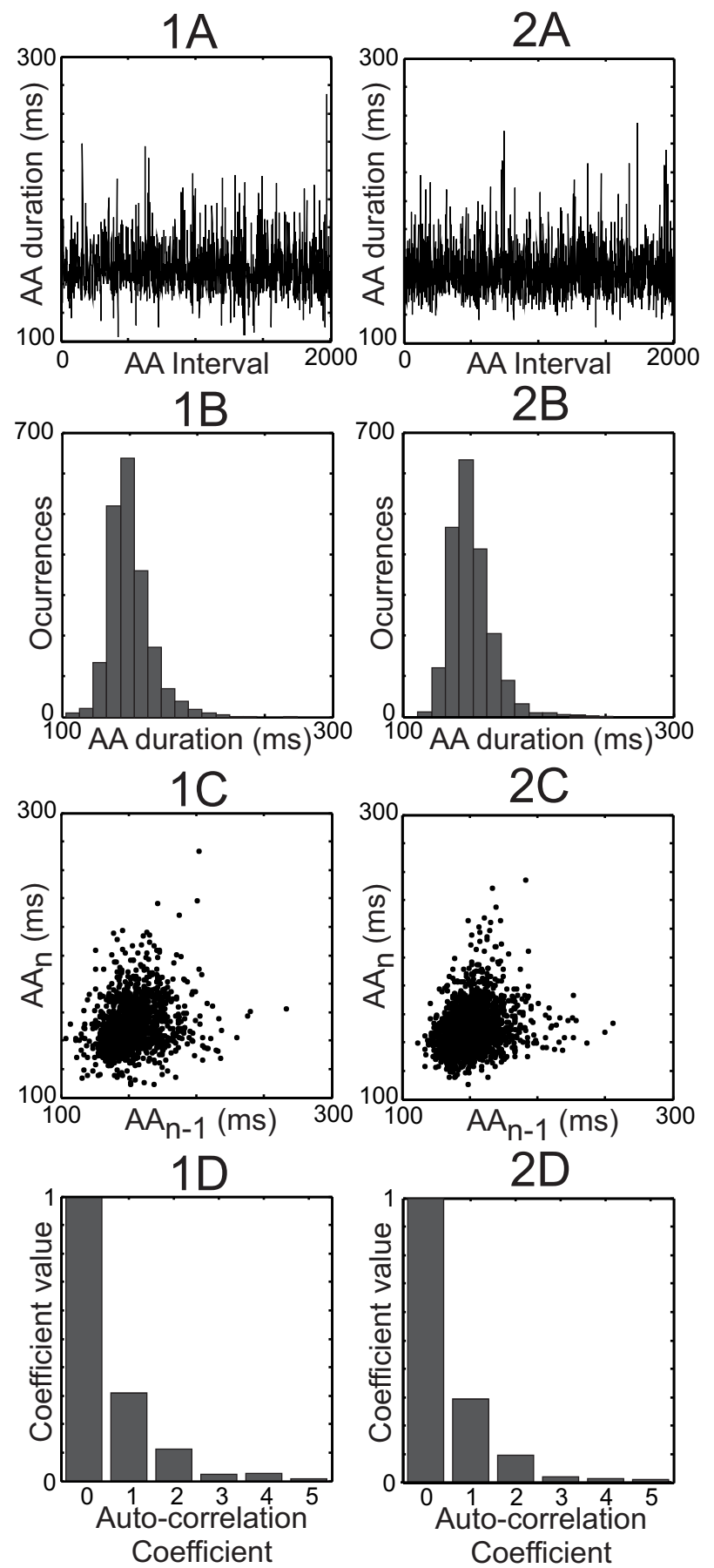

Figure 5.3: Comparison between real (left) and generated (right) AA interval series. AA interval series (A), Standard Histogram (B), Bidimensional Histogram (C), Autocorrelation coefficients (D). 

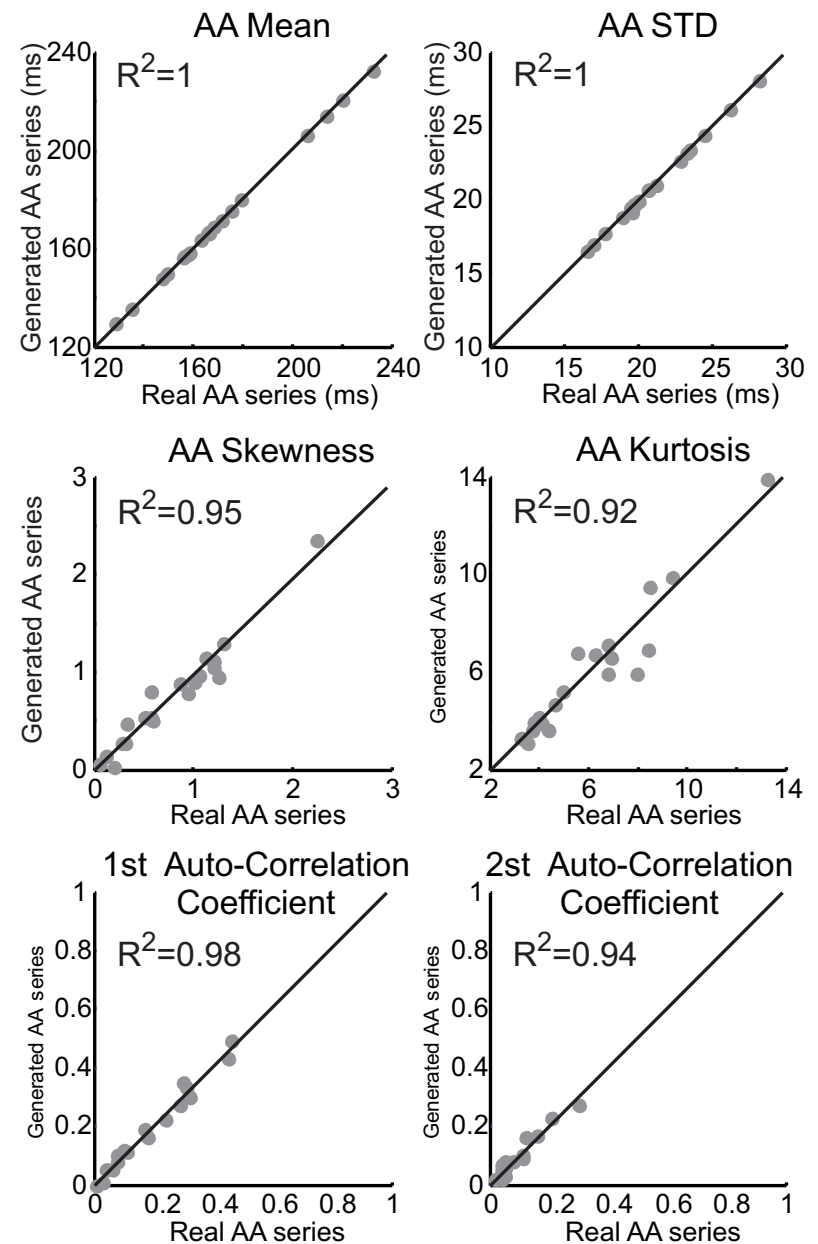

Figure 5.4: Relationship between statistical parameters of recorded AA interval series and generated AA interval series. 
and for an intermediate mean AA value both multiples present a similar number of occurrences. The standard deviation of the AA series is reflected in the width of each RR population, as shown in panel B. In order to increase the values of the standard deviation, the depth of the valley between both RR populations is decreased, and thus identification of both populations is more difficult. Skewness and kurtosis of the AA series also influence the shape of the RR histogram, as can be seen in panels $\mathrm{C}$ and D. Finally, in panel E, the effect of the autocorrelation of the AA interval series in the ventricular response is shown. Therefore, increasing values of autocorrelation are reflected in a decreased valley between both dominant $\mathrm{RR}$ populations.

\subsection{Discussion and Conclusion}

\subsubsection{Main Findings}

This paper develops and presents a novel methodology for the generation of realistic AA interval series during AF. The presented methodology allows the generation of realistic AA interval series with a desired PDF and autocorrelation. We have shown that generated AA series resemble real AA series in terms of their PDF, histogram, Poincaré plot and autocorrelation values.

In addition, we have illustrated the applicability of the present methodology in the study of the ventricular response during AF. We have shown that the characteristics of atrial activity have a great influence on ventricular response. Multiple peaks can be observed in simulated RR histograms, similarly to the RR histogram patterns observed in human recordings. These peaks appeared to be multiples of the mean AA interval and dominance of a particular multiple. The shape of the histogram may also depend on several factors, including the mean atrial rate, its standard deviation, skewness and kurtosis or its autocorrelation.

\subsubsection{Comparison with other studies}

In the literature, several AA series generators have been used with different purposes: testing of $\mathrm{AV}$ node models, generation of RR intervals or simulation of AF in animal experiments [67, 94, 74, 123, 71]. These simple AA generators do not reproduce some statistical properties of real AA series during AF (i.e. shape of the PDF or autocorrelation) and thus their use is limited to certain applications. In particular, they are not well suited for the study of the detailed response of the $\mathrm{AV}$ node during $\mathrm{AF}$, since the conduction properties of the $\mathrm{AV}$ node depend strongly on the history of previously arrived impulses.

The shape of the PDF of AA interval series can be accurately modeled by a Type IV Pearson distribution, which has already been used to approximate the sum of lognormal variables in communication areas [126, 128, 129, 131]. Type IV Pearson distribution is more versatile than other distributions previously used 
A Effect of mean value
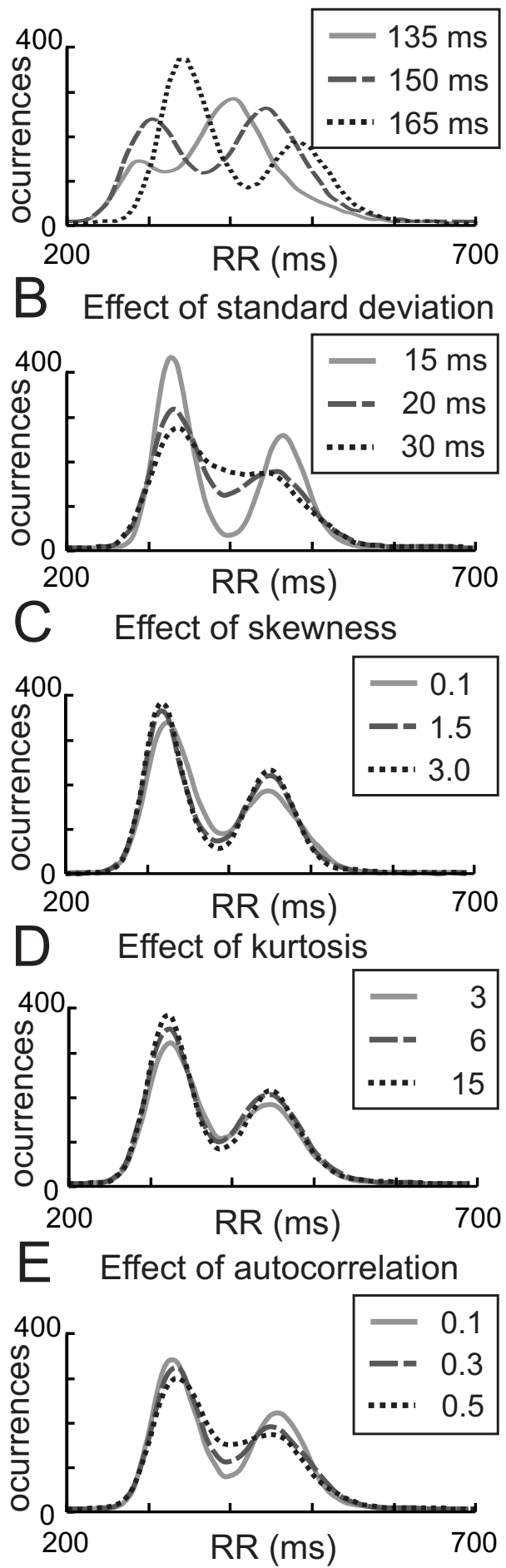

Figure 5.5: RR interval histograms illustrating the role of each statistical parameter of the AA interval series in the ventricular response during AF. The same mathematical model of the AV node was used for all simulations. For the analysis of each parameter (e.g. mean, standard deviation, skewness, kurtosis and autocorrelation) all the other parameters were fixed to mean physiological values parameters (e.g. mean $=155 \mathrm{~ms}$, std $=22 \mathrm{~ms}$, skewness $=1.1$, kurtosis $=8$, autocorrelation coefficients $=\left[\begin{array}{lllll}1 & 0.21 & 0.07 & 0.05 & 0.01\end{array}\right]$ ). (See text for more details). 
for modeling AA interval series and, as expected, has resulted in more accurate results than Gaussian and Poisson PDFs to adjust AA interval series during AF (see Fig.5.2 and Table 5.1). In addition, previously reported, synthetic AA series generators assumed that AA series are uncorrelated, based on the consideration of $\mathrm{AF}$ as a completely random phenomenon [67, 74, 71]. However, different degrees of local and/or transient organization have been observed by us (Fig. 5.4) and some other experimental studies [124, 72, 125]. This temporal organization is reflected in a certain degree of autocorrelation in the series and, as we have shown, it may be of importance to modify the ventricular response.

\subsubsection{Limitations}

In this study, a method for generating synthetic AA interval series is presented. However as with any other model, it is inherently inexact and some limitations should be pointed out: 1) adjustment of the PDF to a Type IV Pearson Distribution requires the condition in Eq. 5.8 to be satisfied. This condition may not apply when attempting to synthesize AA series during organized arrhythmias such as atrial flutter. In this case, a different probability density function should be employed, but the overall methodology may be valid. 2) Dependence of AA intervals on preceding intervals is introduced in an uncorrelated series by applying the filtering method described in Eq. 5.9. This algorithm is appropriate for generating series with a given autocorrelation function with a limited number of non-zero coefficients, such as those observed during AF. However, in cases where a larger number of non-zero autocorrelation coefficients is needed, other methods may be more suitable $[136,122]$. 3) Statistical parameters derived from AA series during $\mathrm{AF}$ have been assumed to be time invariant. However, many authors have reported temporal variations in AA interval series which can be gradual or sudden $[121,137,138]$. These variations could be emulated by concatenating generated AA interval series with different statistical parameters. In fact, an extension of this work might involve the comparison of the presented methodology with longer recordings (i.e. 24 hours) although endocardial or epicardial recordings of more than 5-6 hours are not currently available.

Finally, the mathematical model of the AV node used to evaluate the role of the $\mathrm{AA}$ interval series in the ventricular response during $\mathrm{AF}$ demonstrated its ability to predict AV time in human recordings [73]. However, it does not incorporate some AV characteristics that may play a relevant role in the atrioventricular conduction such as the dual AV node physiology [139]. Since conduction curves measured in previous studies from human AV nodes do not model multiple pathways separately we have introduced the effect of dual AV node physiology in our study. 


\subsubsection{Clinical Applications}

This study presents a versatile methodology for generating realistic AA interval series during AF. For the first time, synthetic AA series present statistical properties (including kurtosis, skewness and autocorrelation) found in real AF recordings. AA series generated by using the present methodology can be applied to the study of the mechanisms related with the ventricular response during $\mathrm{AF}$ and the role of the atrial fibrillatory process in atrioventricular conduction. A deeper understanding of the AV conduction during $\mathrm{AF}$ will help in designing drug therapies for rate control in this group of patients. 


\section{Chapter 6}

\section{Functional Mathematical Model of Dual Pathway AV Nodal Conduction ${ }^{1}$}

\author{
6.1 Introduction \\ 6.2 Materials and Methods \\ 6.3 Results
}

\author{
6.4 Discussion and \\ Conclusions
}

Dual AV nodal pathway physiology is described as two different wavefronts that propagate from the atria to the His bundle: one with a longer effective refractory period (ERP) (fast pathway, FP) and a second with a shorter ERP (slow pathway, SP). By using His electrogram alternance (HEA), we have developed a mathematical model of atrioventricular conduction that incorporates dual AV nodal pathway physiology. Experiments were performed on 5 rabbit atrial-AV nodal preparations to develop and test the presented model. HEAs from the inferior margin of the His bundle were used to identify fast and slow wavefront propagations. The ability to predict AV conduction time and the interaction between FP and SP wavefronts have been analyzed during regular and irregular atrial rhythms (e.g., atrial fibrillation, AF). In addition, the role of dual AV nodal pathway wavefronts in the generation of Wenckebach periodicities has been illustrated. Finally, atrioventricular node ablative modifications have been evaluated. The model accurately reproduced interactions between FP and SP during regular and irregular atrial pacing

\footnotetext{
${ }^{1}$ Chapter based on the manuscript: Andreu M. Climent, M. Salud Guillem, Youhua Zhang, Jose Millet and Todor N. Mazgalev. Functional Mathematical Model of Dual Pathway AV nodal Conduction. Am J Physiol Heart Circ Physiol. 2011 Apr;300(4):H1393-401
} 
protocols. In all experiments specificity and sensitivity higher than $85 \%$ were obtained in the prediction of the pathway responsible for conduction. It has been shown that during AF the SP ablation significantly increased the mean HH interval (204 \pm 39 versus $274 \pm 50 \mathrm{~ms}, \mathrm{p}>0.05$ ), whereas FP ablation did not produce significant slowing of ventricular rate. The presented mathematical model can help in understanding some of the intriguing AV node mechanisms and should be considered as a step forward in the studies of AV nodal conduction.

\subsection{Introduction}

The Atrio-ventricular (AV) node is a small region of the heart that governs the relation between atrial and ventricular activations. During normal sinus rhythm relatively slow conduction along the AV node causes a delay between atrial and ventricular activations and allows an efficient pumping of blood. This reduced conduction velocity is adjusted according to the atrial rate: when the time between two atrial activations (AA) is long, the conduction velocity of the node is higher; when the AA is short, the AV node conduction time increases. This behavior is usually expressed by so-called conduction curve $[76,67,64,65,74,140,73,69,70]$. However, the behavior of the AV node under certain pathological conditions is complex and not well understood. During atrial tachyarrhythmias such as atrial flutter (AFL) or atrial fibrillation $(\mathrm{AF})$ the time between two atrial activations is shorter than the refractory period of AV nodal cells. Consequently, the AV node works as a filter, blocking some atrial activations and limiting the number of ventricular beats. The way in which this natural filter works, and how it could be used to perform efficient ventricular rate control therapies, remains beyond the scope of the simple conduction curve.

One of the intriguing properties of the AV conduction is the so-called dual pathway AV node electrophysiology [23]. This term is used in reference to two different wavefronts that propagate in tandem from the atria to the His bundle $[28,13,23]$, one with a shorter effective refractory period (ERP) and another with a longer ERP (i.e. slow and fast pathways respectively, from now SP and FP). Although this phenomenon was described during 1950s by Moe and collaborators [25, 23], the role that fast and slow wavefronts play in the conduction from the atria to the His bundle still remains debatable. In fact, the evaluation of their individual impact on the AV nodal conduction was ambiguous since the pathway responsible for the conduction of a particular beat could not be identified. Recently Zhang et al. have introduced the His Electrogram Alternance (HEA) [44, 45, 46], a recording technique which allows the identification of the dominant pathway and consequently the evaluation of the role of each pathway during AV nodal conduction.

By analyzing different stimulation protocols along with the HEA, we have 
developed and tested a functional mathematical model of the atrioventricular node that includes dual pathway physiology. In this manuscript the model has been detailed and used to explain some of unclear phenomena related to AV nodal conduction. Specifically, the model has been used to illustrate the role of dual AV nodal pathway wavefronts and their interaction in the generation of paradigmatic Wenckebach periodicities [141, 142, 143]. In addition, the model was used to predict the filtering behavior during irregular atrial rhythms such as AF. Moreover, atrioventricular node modifications (i.e. destructive impairment of conduction by ablation of one of the pathways $[144,145])$, have been modeled and evaluated.

\subsection{Methods}

\subsubsection{In-vitro Experiments}

Experiments were performed on 5 New Zealand White rabbits (Harlan, Indianapolis, IND). Atrial-AVN preparations were instrumented as described previously [44]. Briefly, after anesthesia with sodium pentobarbital $(50 \mathrm{mg} / \mathrm{kg})$, the heart was removed, placed in a glass chamber, and superfused with modified oxygenated Tyrode's solution at $36 \mathrm{C}$, a pH of 7.3 to 7.4 , and a flow rate of $35 \mathrm{~mL} / \mathrm{min}$. After trimming, the final preparation contained the triangle of Koch and the surrounding right atrial and ventricular tissues [44].

Bipolar leads (0.2mm spacing) were custom-made from 125- $\mu \mathrm{m} \mathrm{Ag-AgCL}$ Telfon-isolated wire and used to record atrial electrograms at the crista terminalis and interatrial septum, as well as for atrial pacing. Roving bipolar electrodes were used to record inferior His electrograms as reported previously 12. All electrodes were positioned with micromanipulators (WPI, M330). An 8-channel, programmable stimulator (AMPI, Master-8) was used for pacing. The recorded signals were amplified and filtered at 50 to $3000 \mathrm{~Hz}$ (Axon Instruments, CyberAmp 380), saved on tape (Vetter Digital, 4000A), and later digitized by AxoScope (Axon Instruments) at $5 \mathrm{kHz}$ per channel.

This study was approved by the Institutional Animal Research Committee and is in compliance with the "Guide for the Care and Use of Laboratory Animals" published by the National Institutes of Health (Publication \#85-23, revised 1996).

\subsubsection{Experimental Pacing Protocols}

The time for a given (test) atrial impulse to transverse the node and reach the His bundle (AV nodal conduction time) is primarily dependent on the socalled atrial coupling interval. This is the time interval between the test atrial beat and the beat immediately preceding it. Thus as this coupling interval shortens, the AV conduction time of the test atrial beat prolongs. However, 
this fundamental property is further modified by the impact of pre-preceding atrial beats (so-called conduction history).

In view of such complexity of the conduction properties of the AV node, standardized atrial pacing protocols have been developed and widely used in both experimental and clinical studies of the AV node. One of the most frequently used is the so-called $A_{1} A_{2} A_{3}$ stimulation protocol (Fig. 6.1). It requires the atrium to be paced at a constant base-rate for 10 beats with a fixed coupling interval $A_{1}-A_{1}$ in order to establish a "steady-state" status. After the last beat $A_{1}$ a "conditioning" atrial stimulus $A_{2}$ is introduced (coupling interval $A_{1}-A_{2}$ ), followed finally by the test-stimulus $A_{3}$ (coupling interval $A_{2}-A_{3}$ ). The sequence $A_{1} \cdot A_{1}-A_{2}-A_{3}$ is repeated multiple times, each time with progressively shorter $A_{2}-A_{3}$ coupling intervals.

The atrial electrograms resulting from this pacing protocol are recorded simultaneously with the bundle of His electrograms and the AV nodal conduction time of each atrial beat is determined as $A_{x} H_{x}$, where $\mathrm{x}=1,2$, or 3. The most important variable in this procedure is the test conduction time $A_{3} H_{3}$, which is plotted against the corresponding atrial coupling intervals $A_{2} A_{3}$.

The programmed pacing protocol consisted of construction of four conduction curves $A_{3} H_{3}=f\left(A_{2} A_{3}\right)$ per experiment, each corresponding to one of the 4 conditioning intervals $A_{1} A_{2}$ (used as constant parameters). The $A_{1} A_{2}$ intervals were $300,200,150$ and $125 \mathrm{~ms}$. Thus the pacing paradigm consisted of perpetual (daisy-linked) cycles. Each cycle started with 20 A1A1 beats $\left(A_{1} A_{1}=300 \mathrm{~ms}\right)$, followed by the $A_{1} A_{2}$ beat, followed by the $A_{2} A_{3}$ interval (starting at $A_{2} A_{3}=300 \mathrm{~ms}$ ). The next cycle continued with same 20 $\mathrm{A} 1 \mathrm{~A} 1$ and A1A2 beats, but $A_{2} A_{3}$ was shortened to $295 \mathrm{~ms}$. In this fashion an entire conduction curve was generated until $A_{2} A_{3}$ reached the effective refractory period and AV block ensued. The next conduction curve was generated in similar manner for $A_{1} A_{2}=200 \mathrm{~ms}$, and so-on.

While simpler pacing protocols (for example without the "conditioning" stimulus $A_{2}$ ) have been used for brevity especially in clinical studies, they do not permit proper evaluation of the impact of conduction of earlier atrial beats on the "test" beat. In addition to the programmed $A_{1}-A_{2}-A_{3}$ pacing protocol used to construct AV nodal conduction curves, we have employed fast regular atrial pacing with A-A coupling intervals from $200 \mathrm{~ms}$ to $100 \mathrm{~ms}(300$ to $600 \mathrm{bpm}$ ). This protocol permitted evaluation of Wenckebach periodicity, a conduction pattern where AV nodal conduction time progressively increases from beat to beat until block occurs [146]. Finally, atrial pacing at fast but random rates was used to mimic AV nodal conduction during atrial fibrillation [115].

The above described procedure for generating the conduction curves was completed in 15-20 minutes. The subsequent experimentation (e.g., rapid atrial pacing to induce Wenckebach periodicity or atrial fibrillation, surgical ablations 
A
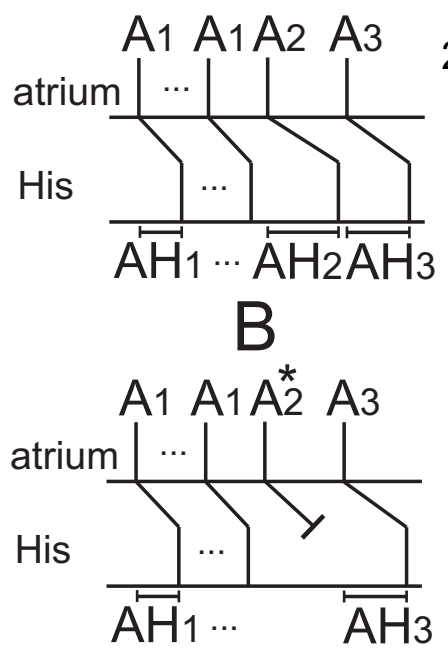

C AV nodal conduction curves

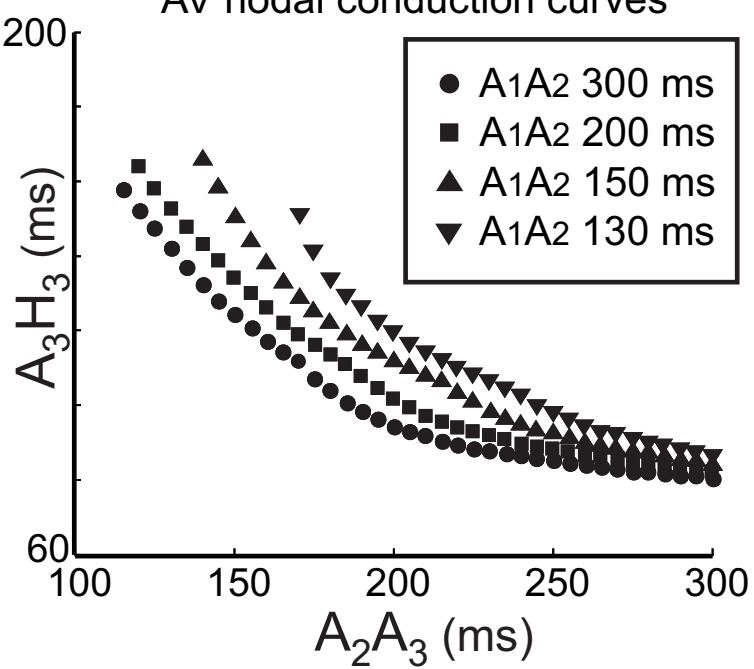

Figure 6.1: Schematic presentation of atrial and ventricular activation periods and the atrioventricular conduction time. In panel $\mathrm{A}$, an $A_{1} A_{2} A_{3}$ stimulation protocol without blocked beats, and in panel $\mathrm{B}$ conduction with a blocked atrial activation $\left(A_{2}{ }^{*}\right)$ are illustrated. The model utilized similar $A_{1} A_{2} A_{3}$ stimulation protocol and was developed from rabbit-heart experimental database and "real" $\mathrm{AV}$ nodal conduction curves $A_{3} H_{3}=\mathrm{f}\left(A_{2} A_{3}\right)$ like those shown in panel $\mathrm{C}$ (rabbit \# 3). The conditioning prematurities A1A2 (300, 200, 250, and $130 \mathrm{~ms}$ ) are shown in the inset. 
described later, etc) was completed in less than 2 hours. At the end, a basic conduction curve was constructed (with $A_{1} A_{2}=300 \mathrm{~ms}$ ) and compared with the initial one to ensure no deterioration of the preparation (e.g., mean conduction time variation $6.7+2.1 \mathrm{~ms})$.

\subsubsection{Differentiation between Conduction Via "Slow" and "Fast" AV Node Pathways}

The phenomenon of His-electrogram alternans has been previously described and validated as a reliable index of dual pathway electrophysiology $[44,45,46]$. Specifically, a characteristic sudden change in the signal amplitude occurs when conduction "switches" from "fast" to "slow" pathway. When bipolar recordings are made from the inferior His bundle domain, the fast pathway (FP) is associated with low-His amplitudes, and pronounced amplitude increase heralds the transition to "slow" pathway (SP) conduction. The change in amplitudes is typically several-fold and can easily be detected when applying either programmed pacing protocols with variable atrial prematurities, or during fast atrial rates including atrial fibrillation. We have used the His-electrogram alternans to assign each conducted beat observed in the rabbit heart experiments to the corresponding AV nodal pathway during programmed pacing, fast atrial rates, or atrial fibrillation.

\subsubsection{Mathematical Model of an AV Nodal Pathway}

The mathematical model developed in this study is based on the rabbit experiments utilizing the above described pacing protocol. The model described below was used for description of either the FP or the SP with constants specific for each of them. Typically, the conduction curve of the intact AV node had been fitted to an exponential function $[67,64,65,74,73,69,70]$. The following expression represents the test conduction time $A_{3} H_{3}$ as a function of the test atrial coupling interval $A_{2} A_{3}$ and the preceding conduction time $\mathrm{A}_{2} \mathrm{H}_{2}$ :

$$
A_{2} H_{3}=A H_{\text {min }}+\beta \cdot \exp \left(-\frac{A_{2} A_{3}-A_{2} H_{2}}{\tau_{\text {rec }}}\right)
$$

where $\mathrm{AH}_{\text {min }}$ is the minimum observed time for an atrial activation to reach the His bundle, observed at very slow atrial rates; $\tau_{\text {rec }}$ is AV nodal recovery period, which is related to the effective refractory period of the nodal cells; $\beta$ is a modulating factor.

The recovery period $\tau_{r e c}$ is not set to a constant value since the effective refractory period of nodal cells depends on the activation rate [147]. The recovery period was modeled by the following expression:

$$
\tau_{\text {rec }}=\gamma_{1}+\left(\frac{A_{2} H_{2}}{\gamma_{2}}\right)^{\gamma_{3}}
$$


where $\gamma_{1}, \gamma_{2}$ and $\gamma_{3}$ are constants whose values are different for each pathway. Finally, the modulating factor $\beta$ was modeled as follows:

$$
\beta=\lambda_{1} \cdot\left(A_{2} H_{2}\right)^{2}+\lambda_{2} \cdot A_{2} H_{2}+\lambda_{3}
$$

where $\lambda_{1}, \lambda_{2}$ and $\lambda_{3}$ are constants which depend on the pathway. Although previous studies have proposed a formulation of the $\beta$ modulation factor as dependent only on the base rate $A_{1} A_{2}$ [70], the formulation proposed in our model fitted better to the experimental data, in part because conduction history (including both $A_{1} A_{1}$ and $A_{1} A_{2}$ coupling intervals) is directly reflected in conduction time $\mathrm{A}_{2} \mathrm{H}_{2}$.

\subsubsection{Incorporation in the Model of "Concealed" Conduction}

Equations 6.1-6.3 are used as a first estimate of the conduction time for an atrial stimulus through a single AV nodal pathway. However, if the estimated conduction time is longer than a physiological maximum conduction time $\left(A H_{\max }\right)$, then the pathway is considered blocked. The parameter $A H_{\max }$ was specific for each pathway and was derived from the experimental data during fast atrial rates as the longest $\mathrm{AV}$ conduction time associated with either the FP or the SP.

When an atrial stimulus fails to be conducted it is marked $A_{2} *$ (Fig. 6.1B). Its effect on the test beat $A_{3}$ depends on the degree of penetration (concealment) of the $A_{2}{ }^{*}$ beat into the pathway. Specifically, if the propagation wavefront initiated by $A_{2} *$ is blocked near the atrial (proximal) side of the pathway, its effect on the subsequent conduction time $A_{3}-H_{3}$ is lesser than if $A_{2}{ }^{*}$ has been blocked distally (closer to the bundle of His). This postulate reflects an important AV nodal physiological property during "concealed" conduction: a proximal $A_{2}{ }^{*}$ block, at the same value of $A_{2}{ }^{*} A_{3}$, permits longer diastolic pause and thus fuller recovery for all distal cells, which leads to relatively better (faster) conduction $A_{3} H_{3}$ compared to the case of a deeper $A_{2}{ }^{*}$ penetration (or ultimately a full $A_{2} H_{2}$ conduction).

In the model, the degree of $A_{2}{ }^{*}$ penetration is computed by using equations 6.1-6.3, where the activation time $A_{2} H_{2}$ is replaced by a "virtual" conduction time $\mathrm{A}_{2}{ }^{*} \mathrm{H}_{2}$ :

$$
A_{2} H_{2} \rightarrow A_{2} * H_{2}=c_{1}-c_{2} \cdot \exp \left(-\frac{A_{1} A_{2}^{*}}{c_{3}}\right)
$$

where $c_{1}, c_{2}$ and $c_{3}$ are constants and $A_{1} A_{2}{ }^{*}$ is the coupling interval for the blocked beat $A_{2} *$ (Fig. 6.1B). The term "virtual" indicates that, in fact, there is no $H_{2}$ electrogram since $A_{2}{ }^{*}$ is a blocked beat. However, as it follows from eq. 6.4 , a longer $A_{1} A_{2} *$ coupling interval (associated with deeper penetration) 
results in larger $A_{2} * H_{2}$ value, which in turn (see eq. 6.1) leads to longer test conduction time $\mathrm{A}_{3} \mathrm{H}_{3}$.

\subsubsection{Description of the Dual Pathway Structure}

For each rabbit, both FP and SP AV nodal conduction were modeled using equations 6.1-6.4 with constants fitted for each pathway. In this manner, two different $A_{3} H_{3}$ conduction time functions were computed: $A_{1} A_{3}^{F P}$ and $A_{1} A_{3}^{S P}$. As illustrated in Fig. 6.2, for modeling purposes we used a simple Y-shaped structure to represent the SP and FP starting at the atrium and converging into a "final common pathway" that reaches the bundle of His. Such schematic presentation, while admittedly simplistic, is very close to the original description of the rabbit dual pathway physiology $[25,23]$ and permits an easy differentiation: the model "decides" which is the dominant pathway by determining the shorter of the two conduction times $A_{1} A_{3}^{F P}$ and $A_{1} A_{3}^{S P}$ for a specific atrial beat. Depending on atrial coupling intervals sequence $A_{1}-A_{2}-A_{3}$, the leading wavefront can be the FP (Fig. 6.1A, D), or the SP (Fig. $6.1 \mathrm{~B}, \mathrm{C}$ ). In the real rabbit experiments the presence of $\mathrm{FP}$ or $\mathrm{SP}$ conduction is confirmed by the His electrogram alternans (Fig. 6.2): His electrograms with high-amplitudes indicate SP conduction, whereas low-His amplitudes are associated with FP conduction.

As evident from the Y-structure (Fig. 6.2), the leading wavefront could retrogradely invade the pathway with the longer calculated $A_{3} H_{3}$ (Fig. $6.2 \mathrm{~A}$, B), thus colliding with and annihilating the later wavefront. A wavefront can also be blocked in either pathway at an atrial coupling interval encroaching on the pathway's effective refractoriness (Fig. 6.2 D, E). After a complete block of the AV node, the conduction pattern in the subsequent beat would depend on the degree of penetration of each wavefront in the corresponding pathway, as modeled by eq. 6.4. Accordingly, each of the scenarios shown in Fig. 6.2 A-E may be possible for the beat following an AV nodal block.

\subsubsection{Calculation of Model's Constants}

Constants of the model were determined for each of the dual pathways by using the experimental AV nodal conduction curves as well as the data obtained with regular atrial pacing. All experimentally observed conduction times were considered in tandem with the His electrogram of the corresponding beat in order to delineate conduction via the FP or SP based on His alternans (Fig. 6.3). Thus, $A H_{\text {min }}$ of the AV node was defined as the minimum AH time typically observed at base-rate pacing. $A H_{\max }$ of the FP and $\mathrm{SP}$ was defined as the maximum AV nodal time measured during rapid atrial rate stimulation protocol, and associated with low and high His amplitudes, respectively. Similarly, the parameters $\beta$ and $\tau_{\text {rec }}$ were estimated for each pathway by using the corresponding portions of the experimental 


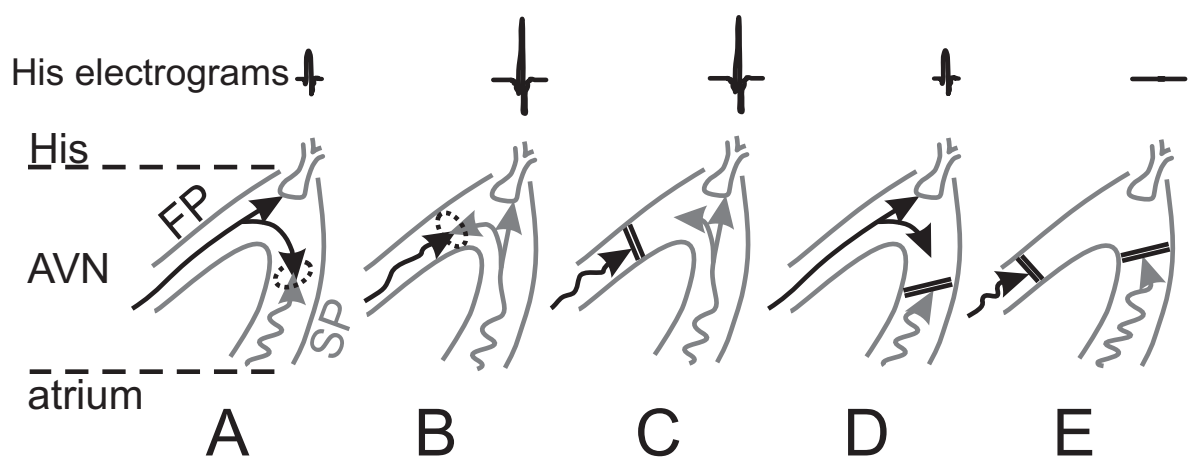

Figure 6.2: Illustration of different conduction interaction scenarios between fast and slow pathway propagations in a simplified Y-shaped atrial-AVN-His structure. Black arrows indicate fast pathway (FP) whereas gray arrows indicate slow pathway (SP) wavefront. In panel A, typically at long atrial coupling intervals, FP wavefront activates the His bundle and a retrograde wavelet meets and blocks the later SP wavefront. Similarly, in panel B, typically at short atrial prematurities, SP conduction reaches the His bundle and a retrograde wavelet blocks the later FP. In panel C, the FP wavefront is stopped upon encountering refractory tissue whereas the $\mathrm{SP}$ wavefront reaches the His bundle. In panel D, a similar situation is depicted for the SP wavefront. In panel E, both FP and $\mathrm{SP}$ wavefronts are blocked due to refractoriness at atrial prematurity defined as AV node effective refractory period. 
conduction curve activations with either low or high His electrogram. Since a family of conduction curves was generated with different conditioning cycle length $A_{1} A_{2}$, a large-scale curve fitting algorithm based on the interior-reflective Newton method $[148,149]$ was used to estimate $\beta$ and $\tau_{r e c}$, thus having as many values of $\beta$ and $\tau_{\text {rec }}$ as different $A_{1} A_{2}$ cycles were used. For each pathway, computed $\beta$ values were then used to estimate $\lambda_{1}, \lambda_{2}$ and $\lambda_{3}$ constants in eq. 6.2 whereas computed $\tau_{\text {rec }}$ values were used to estimate $\gamma_{1}$, $\gamma_{2}$ and $\gamma_{3}$ constants in eq. 6.3.
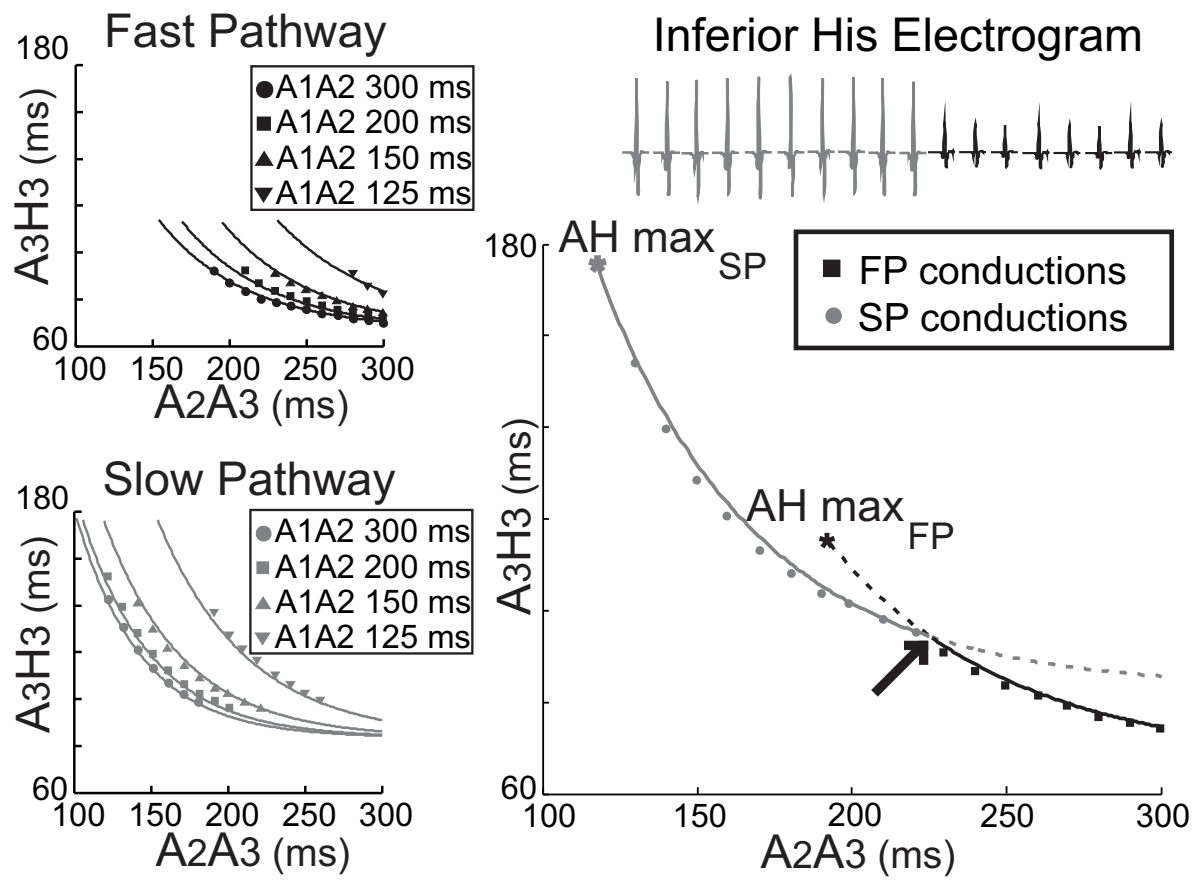

Figure 6.3: In the left panels model conduction curves for FP (black lines) and SP (gray lines) conduction with different $A_{1} A_{2}$ conditioning intervals (inset) are shown based on data points (dots) derived from rabbit \# 2. In the right panel a composite conduction curve for the intact $\mathrm{AV}$ node is shown $\left(A_{1} A_{2}=150 \mathrm{~ms}\right)$. Note that experimental data points are assigned to FP (black) and SP (gray) according to His-alternans index: the FP conduction is associated with lowamplitude His electrograms, while SP conduction results in high-amplitude His electrograms (see top trace). The transition from FP to SP was observed at $A_{2} A_{3}=226 \mathrm{~ms}$ (arrow).

Protocols with fast regular atrial pacing rate that lead to AV blocks but at the same time resulted in exclusive either FP or SP propagation were used to estimate constants in eq. 6.4, which models the concealed conduction. For each 
experimental case, the $A_{3} H_{3}$ conduction time measured for the beat following a blocked stimulus was used to estimate the "virtual" conduction time $A_{2}{ }^{*} H_{2}$ that would have resulted in the measured $A_{3} H_{3}$ according to eq. 6.1. The calculated $A_{2}{ }^{*} H_{2}$ values were then used to fit the constants $c_{1}, c_{2}$ and $c_{3}$ (eq. 6.4 ) by least squares fitting. This procedure was applied separately for each pathway.

\subsubsection{Validation of the Model}

In order to determine the fitting quality of the model, the protocols used during the rabbit experiments were reproduced in the model. Root mean squared error was measured to estimate the difference between experimental and modeled conduction curves. This test showed, in effect, how well the equations and the calculated model constants reflect the experimental database.

In order to validate the functional performance of the model we executed two additional tests, independently in the animal preparations and in the AV nodal model. First, atrial pacing was used that, within a range of AA intervals, produced Wenckebach periodicity in the animal experiments with conduction pattern indicating FP and SP conduction alternations. The same protocol was then applied to the model. Second, a sequence of 200 irregularly distributed atrial AA intervals were used for pacing that mimics atrial fibrillation. In both cases, we established the goodness of the model to predict the AV nodal pathway used for each atrial beat as well as the value of the experimentally measured conduction times.

\subsection{Results}

\subsubsection{Conduction Curve Fitting and Model Equation Constants}

In all rabbits, depending on atrial coupling intervals, two distinct (low and high) amplitudes, were detected in the inferior His electrogram recordings identifying the FP and SP conduction, respectively (Fig. 6.3, right panel). Therefore, both pathways could be modeled in each experiment. Constants of the model were calculated for each rabbit heart and are summarized in Table 6.1. As expected, $A H_{\min }$ and $A H_{\max }$ of the FP were always shorter than the $A H_{\min }$ and $A H_{\max }$ of the $\mathrm{SP}$, respectively.

As seen in Fig. 6.3 from one rabbit heart, the model-derived conduction curves (left panels, solid lines) closely approximated the experimentally observed data points. The model-generated curves based on data for the FP (corresponding to low-His amplitudes) are shown in black, and those for the SP (corresponding to high-His) are in gray. Each pathway was modeled as an exponential curve and the transition from FP to SP conduction is clearly 
Table 6.1: Values of constants for the models of FP and SP in each rabbit heart.

\begin{tabular}{|c|c|c|c|c|c|c|c|c|c|c|c|}
\hline \multicolumn{12}{|c|}{ FAST PATHWAY } \\
\hline Rabbit & $A H_{\min }$ & $A H_{\max }$ & $\lambda_{1}$ & $\lambda_{2}$ & $\lambda_{3}$ & $\gamma_{1}$ & $\gamma_{2}$ & $\gamma_{3}$ & $c_{1}$ & $c_{2}$ & $c_{3}$ \\
\hline 1 & 61 & 87 & 0,001 & $-0,31$ & 163 & 83 & 107,7 & 61,85 & 189 & 165 & 116 \\
\hline 2 & 67 & 92 & 0,009 & $-2,82$ & 339 & 48,3 & 10 & 1,31 & 295 & 20 & 185 \\
\hline 3 & 66 & 108 & 0,013 & $-2,76$ & 335 & 56,28 & 102,3 & 8,33 & 161 & 5334 & 22 \\
\hline 4 & 77 & 115 & 0,019 & $-5,87$ & 622 & 66,48 & 92,3 & 3.35 & 502 & 632 & 183 \\
\hline 5 & 57 & 79 & 0,001 & 0,07 & 75 & 75,67 & 186 & 54,46 & 320 & 492 & 94 \\
\hline \multicolumn{12}{|c|}{ SLOW PATHWAY } \\
\hline Rabbit & $A H_{\min }$ & $A H_{\max }$ & $\lambda_{1}$ & $\lambda_{2}$ & $\lambda_{3}$ & $\gamma_{1}$ & $\gamma_{2}$ & $\gamma_{3}$ & $c_{1}$ & $c_{2}$ & $c_{3}$ \\
\hline 1 & 83 & 175 & 0,2 & $-42,98$ & 2466 & 25,76 & 56,38 & 3,72 & 172 & 362 & 96 \\
\hline 2 & 86 & 182 & 0,121 & $-28,55$ & 1826 & 32,23 & 51,67 & 1,8 & 195 & 20 & 185 \\
\hline 3 & 83 & 171 & 0,046 & $-10,43$ & 688 & 37,93 & 34,62 & 2,23 & 165 & 19140 & 18 \\
\hline 4 & 97 & 178 & 0,005 & $-1,7$ & 244 & 39,25 & 164 & 43,35 & 181 & 130000 & 8 \\
\hline 5 & 69 & 135 & 0,1 & $-23,09$ & 1611 & 27,11 & 50 & 1,13 & 280 & 492 & 94 \\
\hline
\end{tabular}

All constants were determined by using the experimental pacing protocols and data (see text for more details).

indicated upon superposition (right panel) by the crossover of the 2 curves at some critical prematurity $A_{2} A_{3}$ (arrow). For longer $A_{2} A_{3}$ the FP was "predominant" since its conduction times were shorter. In contrast, for shorter $A_{2} A_{3}$, the shorter SP conduction times determined its predominance. In humans the transition between FP and SP conduction is frequently (although not always) associated with a characteristic interruption ("jump") in the conduction curve $[150,24]$. In the rabbit heart the conduction curves are rather smooth, although the transition between the dual pathways could still be visually detected (Fig. 6.3, right panel).

We have model-generated FP and SP curves for each rabbit heart using identical pacing protocols with the real animal experiments. Typically 4 pairs of conduction curves (corresponding to the conditioning $A_{1} A_{2}$ prematurities) were generated for each heart (Fig. 6.3, left panels) and then superimposed to obtain the conduction curve of the "intact" AV node (one such superposition for $A_{1} A_{2}=150 \mathrm{~ms}$ is illustrated in Fig. 6.3, right panel). For all studied rabbit hearts the root mean squared error of the FP and SP conduction times were $7 \pm 4 \mathrm{~ms}$ and $3 \pm 3 \mathrm{~ms}$ respectively. The $A_{2} A_{3}$ interval at which conduction changed from FP to SP (Fig. 6.3, right panel, arrow) measured by the model coincided with that obtained in the real experiments.

\subsubsection{Validation of the Model in Predicting Wenckebach Periodicity: Comparison with Rabbit AV Node}

In Fig. 6.4A an example of regular atrial pacing at $160 \mathrm{~ms}$ that generate Wenckebach periodicity and irregular $\mathrm{HH}$ intervals series for rabbit \# 1 is 
illustrated. In this case, a 5:4 Wenckebach behavior is present (i.e. four out of five atrial activations are conducted and one is blocked). The AV nodal conduction times within the Wenckebach cycle progressively increased (110ms, $132 \mathrm{~ms}, 143 \mathrm{~ms}$ and $153 \mathrm{~ms}$ ) until the occurrence of block. Importantly, as indicated by the amplitudes of the recorded His electrograms, the 1st atrial stimulus after AV block was conducted via the FP (low-His) whereas the 2nd, 3rd and 4th atrial stimulus were conducted via the SP wavefront (high-His). By applying the same atrial pacing series to the model, both the utilized pathway and the correct AV nodal times were faithfully reproduced (panel A, bottom trace indicates FP and SP conducted beats by short and longer bars, respectively).

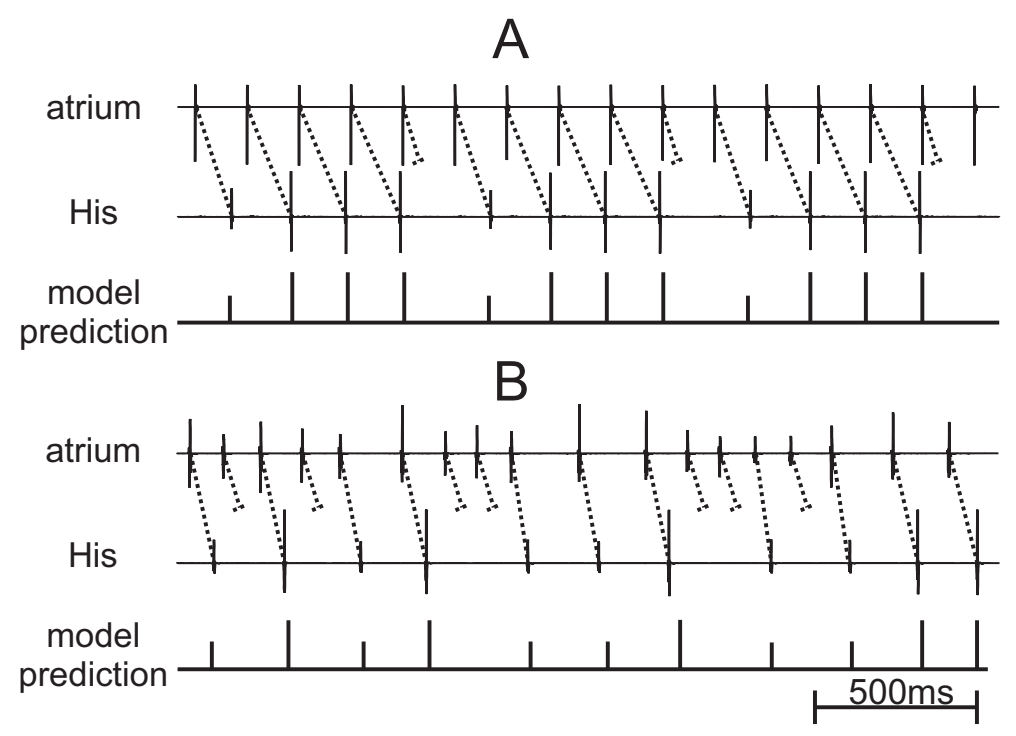

Figure 6.4: Panel A, schematic depiction of atrial and His electrograms recorded during regular atrial pacing intervals that generated Wenckebach phenomenon in an intact AV node (data from rabbit \# 1). Note His-amplitude alternans indicative for presence of FP and SP conduction. Panel B, atrial and His electrograms during random atrial pacing in the same heart that generated irregular atrioventricular conduction times (and therefore irregular His distribution) with alternation between FP and SP conduction. In both panels the predictions of the model for the same atrial pacing series are illustrated in the traces below the real His electrograms.

An average of $17 \pm 3$ Wenckebach recordings were obtained in each rabbit experiment with a constant-rate atrial pacing with atrial coupling intervals between $158 \pm 22$ and $134 \pm 19 \mathrm{~ms}$. In each experiment, in at least 3 Wenckebach series (average $5 \pm 2$ ) there was evidence for conduction 
alternating between the FP and SP. When the same AA interval series were applied to the model of each corresponding rabbit AV node, the correct AV node pathway used in a particular beat of the Wenckebach series was predicted $100 \%$ of times, and the root mean square errors of the calculated conduction times via the FP and SP were $9 \pm 5 \mathrm{~ms}$ and $14 \pm 9 \mathrm{~ms}$, respectively.

\subsubsection{Validation of the Model in Predicting Atrial Fibrillation: Comparison with Rabbit AV Node}

In Fig. 6.4B, the participation of AV nodal dual pathways during an episode of AF-like random atrial stimulation is illustrated (rabbit \# 1). As seen from the figure, the His electrogram alternans indicate that during irregular AA intervals some beats propagated via the FP (low-His) while others utilized the SP (highHis). Just like the randomness of the atrial beats, the FP and SP wavefront occurred in a random order. This in part was reflected in the observation that long (as well as short) HH intervals could terminate with either FP or SP Hiselectrograms. In other words, it was not possible to deduct which pathway was responsible for the conduction of a particular atrial beat by analysis of $\mathrm{HH}$ intervals duration alone. Therefore, in general, the assumption that long $\mathrm{HH}$ are due to FP conduction and short $\mathrm{HH}$ intervals are due to SP conduction [40] may be incorrect. As it can be seen from Fig. 6.4B, the model estimation of the involvement of dual AV nodal pathways during AF was highly accurate in this experiment.

Similar percentage of $\mathrm{FP}$ and $\mathrm{SP}$ conductions during $\mathrm{AF}$ was found in 4 of the 5 cases, and only in one case we observed almost-exclusive SP conduction. Table 6.2 summarizes mean values of sensitivity and specificity of the mathematical model in the prediction of the AV node response for a series of 200 random AA interval series. In all cases a specificity and sensitivity higher than $85 \%$ were obtained. Although SP conduction was more frequent (44\%), the number of FP and blocked beats (i.e. $29 \%$ and $27 \%$, respectively) was significant during AF.

Table 6.2: Accuracy of model predictability for presence of FP and SP conduction during atrial fibrillation.

\begin{tabular}{lccc}
\hline \hline & $\mathrm{N}$ & Specificity & Sensitivity \\
\hline FP conduction & $57 \pm 21$ & $96 \pm 2 \%$ & $89 \pm 6 \%$ \\
SP conduction & $89 \pm 15$ & $93 \pm 3 \%$ & $88 \pm 5 \%$ \\
Blocked conduction & $54 \pm 9$ & $93 \pm 3 \%$ & $91 \pm 9 \%$ \\
\hline
\end{tabular}

N, number of beats. FP, Fast Pathway. SP, Slow Pathway. 


\subsubsection{Model Predictions after Modifications of Dual Pathway Electrophysiology}

The AV nodal dual pathway electrophysiology is the basic mechanism underlying the complexity of atrio-ventricular conduction and plays an important role in the genesis and maintenance of various arrhythmias $[151,152]$. Since the Ystructure (see Fig. 6.2) of the atrial-ventricular connections could naturally sustain re-entrant excitation, specific ablation procedures have been developed clinically to modify dual pathways by impairing one or the other in an attempt to interrupt the re-entrant circuit.

Since the ablation procedure is destructive and irreversible, experiments in real hearts could employ either SP or FP modification, thus limiting an indepth study of AV nodal properties. The model offers the unique opportunity to study the same AV node upon "ablation" of either pathway in any order, and to elucidate the conduction pattern during complex arrhythmias induced by unlimited choice of pacing algorithms.

\subsubsection{Selective AV Nodal Ablations and Wenckebach Periodicity}

Fig. 6.5 depicts an example in which a 5:4 Wenckebach periodicity was first generated in the intact AV node model (panel A). By sequentially "ablating" in the model either the SP or the FP, the conduction patterns observed with only FP (panel B) or SP (panel C) could be delineated. Note that in all cases, as should be expected during Wenckebach periodicity, there was a progressive increase of the conduction times $\mathrm{AH}$ in the cycle. However, the evolution of HH-intervals was different. Within the Wenckebach cycle (i.e., before the blocked beat) there was progressive prolongation of $\mathrm{HH}$ in the intact AVN and in the AVN with only-SP (panels A and C, upward arrows), but decreasing $\mathrm{HH}$ were present with only-FP (panel B, downward arrows). The latter behavior is known clinically as "typical Wenckebach" and the former is described as "atypical Wenckebach" [153]. While the specific mechanism(s) for such conduction peculiarity is not well understood, our model suggests that the involvement of AVN dual pathways electrophysiology could be a likely explanation. In fact, the model predicts that even small changes in atrial rate can alter significantly the pattern of Wenckebach periodicity. Thus Fig. 6.6 (bottom panels, curved arrows) shows that at an atrial coupling interval of 158 ms the intact node can exhibit both a "typical" and "atypical" pattern of $\mathrm{HH}$ evolution.

We ran all 5 rabbit heart models through a wide range of atrial pacing rates both in intact and ablated (FP or SP) modes and were able to generate both "typical" and "atypical" Wenckebach in each case. Some summarized results are shown in Fig. 6.7 and demonstrate that during Wenckebach periodicity the properties of the intact AV node were mostly determined by 


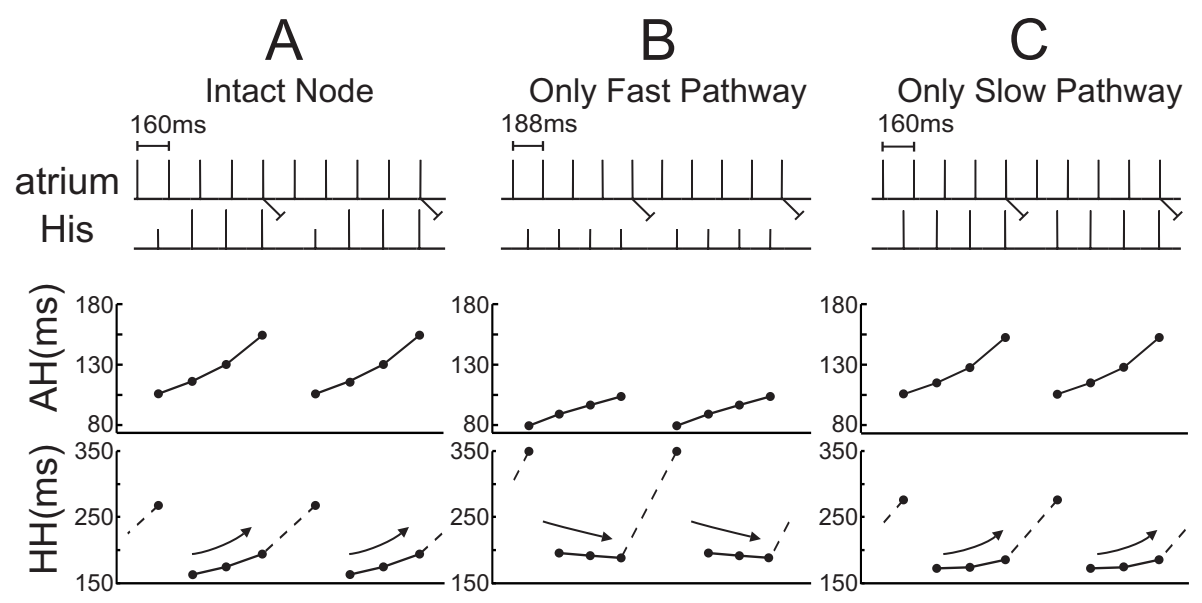

Figure 6.5: An example of 5:4 Wenckebach periodicity during regular atrial pacing in an intact AV node model (column A, rabbit heart \# 1), and modelderived sequences with only-FP (column B) and only-SP (column C) conduction. The upper panels schematically represent atrial and His electrograms. The Hamplitude alternans indicates presence of specific pathway: FP (low amplitude), or SP (high amplitude). The middle panels show the AH conduction times of consecutive beats in the Wenckebach cycle, whereas the bottom panels represent the $\mathrm{HH}$ interval evolution. Blocked atrial beats are marked by the symbol $\perp$ (upper panels) and by dashed lines in the $\mathrm{HH}$ panels. Upward arrows indicate "atypical" Wenckebach pattern with progressively longer $\mathrm{HH}$, while downward arrows indicate "typical" pattern with progressively shorter $\mathrm{HH}$ intervals in the Wenckebach cycle. See the text for details. 


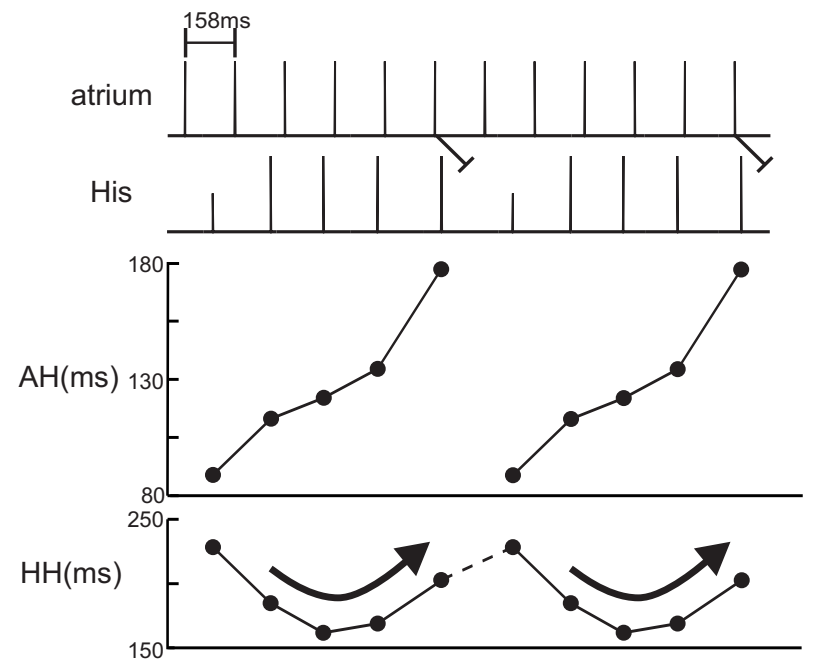

Figure 6.6: An example of 6:5 Wenckebach periodicity during regular atrial pacing in an intact AV node (data from rabbit \# 4). The figure is organized similarly to Fig. 6.5. Note the presence of both typical and atypical HH pattern (curved arrows). See the text for details.

the presence of the SP. When compared to only-FP conduction (columns "c"), both the intact node (columns "a") and only-SP conduction (columns "b") revealed Wenckebach periodicity at relatively shorter AA intervals that also occupied a wider range. The predominant role of the SP can also be derived from all traces in Figs. 6.4, 6.5, and 6.6 (intact AVN) whereas FP conduction was only observed in the first beat of the Wenckebach cycle with all subsequent beats utilizing the SP.

\subsubsection{Selective AV Nodal Ablations and Atrial Fibrillation}

In Fig. $6.8 \mathrm{HH}$ intervals resulting from random AA pacing (containing intervals from 75 to $125 \mathrm{~ms}$ ) are depicted in one modeled heart. Table 6.3 summarizes the shortest, the average and longest $\mathrm{HH}$ intervals in all 5 rabbit heart models using intact, only-SP, or only-FP models. Ablation of the FP (Fig. 6.8, middle panel) did not produce significant prolongation in the mean $\mathrm{HH}$ interval (a measure for slowing of ventricular rate). This finding agrees with both experimental $[44,45,46]$ and clinical $[144,145]$ observations indicating relatively low effectiveness of FP-ablations as a therapy to control (i.e., to slow) ventricular rate in AF. In contrast, ablation of the SP (right panel C) produced a significant reduction in the number of short $\mathrm{HH}$ intervals, resulting in an increase of the minimum $\mathrm{HH}$ interval, as well as a prolongation 


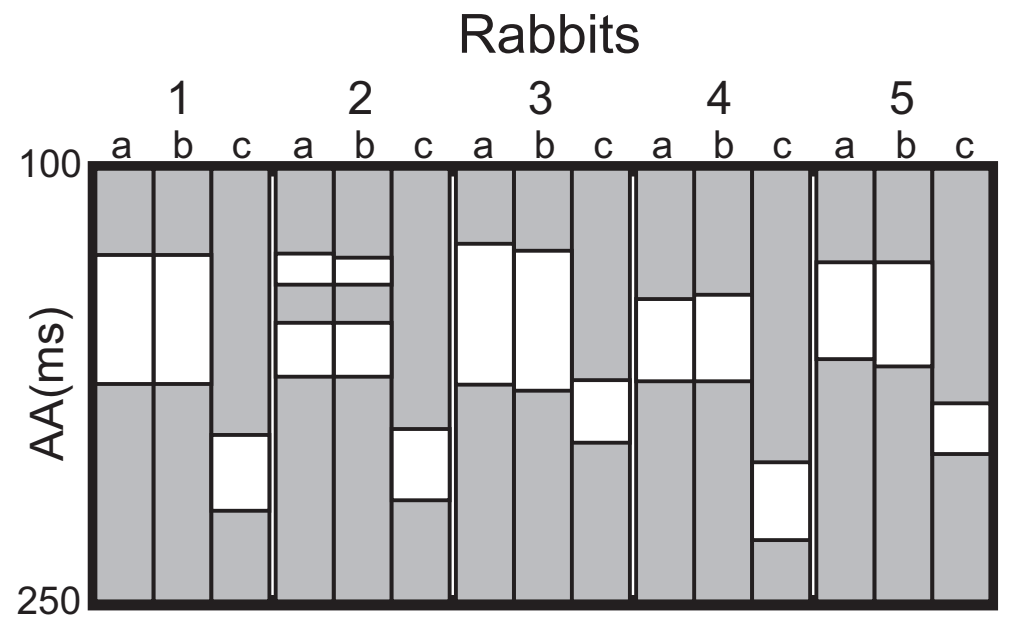

Figure 6.7: Systematic analysis of the atrial AA pacing intervals producing Wenckebach periodicity in intact AV models, and after selective SP or FP ablation. The white bars indicate ranges of AA intervals for which the Wenckebach phenomenon was generated in the intact AV node (a), in only the SP (b) and in only the FP (c). As seen, FP ablation (panels b) did not modify significantly AA interval ranges producing Wenckebach periodicity in the intact node. However, after SP ablation (panels c) Wenckebach periodicity was observed at longer AA intervals that occupied narrower range. This suggests that Wenckebach-like conduction patterns in the intact AV node are predominantly determined by the SP. 
of the average $\mathrm{HH}$ interval. The increase in the average $\mathrm{HH}$ interval after $\mathrm{SP}$ ablation was highly correlated with the ratio between the number of SP and FP conducted beats in the intact heart $(\mathrm{R}=0.91, \mathrm{p}>0.05)$. Thus the model predicts that SP-ablation, which is a common clinical procedure for control of ventricular rate, $[144,145]$ should be expected to be particularly effective in intact hearts that favor SP conduction during AF. The latter could be determined by applying the His-alternans index before performing the ablation procedure (as was done in the left panel of Fig. 6.8).

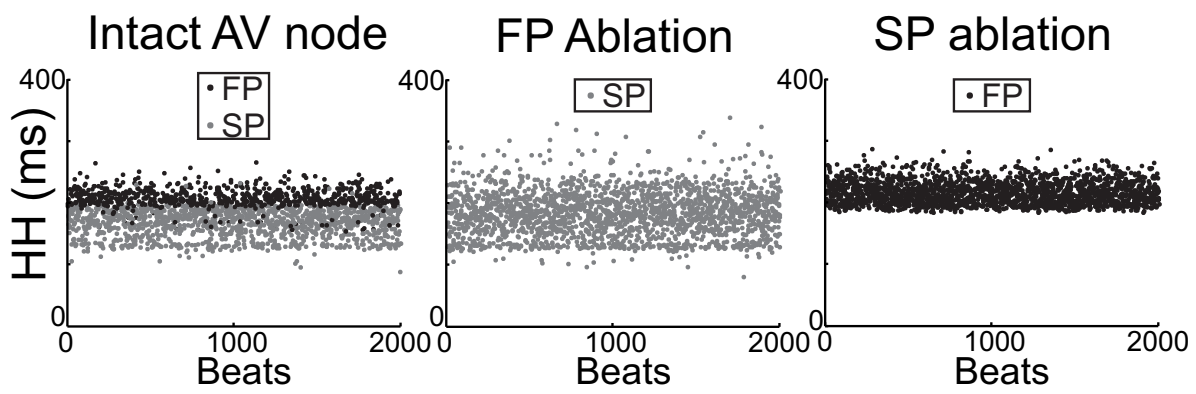

Figure 6.8: Distribution of 2000 consecutive $\mathrm{HH}$ intervals during random atrial pacing (AA intervals from 75 to $125 \mathrm{~ms}$ ) in the intact node (left), after FPablation (middle) and after SP-ablation (right). Data was derived from the model of rabbit heart \# 2. Beats conducted via the SP are shown as grey dots, and beats conducted via the FP are shown as black dots. See the text for details.

Table 6.3: Effect of selective pathway ablation on $\mathrm{HH}$ intervals during atrial fibrillation.

\begin{tabular}{lccc}
\hline \hline & Shortest HH $(\mathrm{ms})$ & Average HH $(\mathrm{ms})$ & Longest HH (ms) \\
\hline Intact AV node & $125 \pm 29$ & $202 \pm 39$ & $318 \pm 50$ \\
FP-ablation & $127 \pm 30$ & $205 \pm 41$ & $436 \pm 72^{*}$ \\
SP-ablation & $179 \pm 72^{*}$ & $274 \pm 50^{*}$ & $401 \pm 47^{*}$ \\
\hline
\end{tabular}

$* \mathrm{p}>0.05$ respect to control values

\subsubsection{Multimodal HH interval Histograms: Role of the Dual AV Nodal Physiology}

As commented previously in the present thesis, the existence of bimodal $\mathrm{HH}$ histograms has been used in the literature as an indirect identification of dual AV nodal physiology [40, 97, 43]. However, as we previously demonstrated [109], the presence of more than one predominant $\mathrm{HH}$ distribution may be due mainly to different AV nodal conduction patterns (2:1, 3:1, 4:1, etc.) rather than FP 
or SP conductions. In order to clarify these phenomena, different experiments were developed by using the mathematical model of the AV node presented in this chapter and the AA intervals series generator presented in chapter 5 . These simulation produced different ventricular response patterns depending on the relationship between the atrial signal and atrioventricular characteristics. About the bimodal HH histogram phenomenon, all different ventricular response patterns can be summarized in four scenarios that are illustrated in (Fig. 6.9).

In the first scenario (panels 1A to 1D) an example for rabbit 4 is illustrated. The intact AV node presented a unimodal histogram although both FP and SP conductions were present (panel 1B). The mean AA interval in this case was $110 \mathrm{~ms}$ and the most probable $\mathrm{HH}$ interval was around $220 \mathrm{~ms}$ which may represent a predominant 2:1 conduction pattern. When a simulated ablation of the SP (panel 1C) or the FP (panel 1D) was produced, no significant differences were observed in the ventricular response pattern since unimodal histograms were present.

In the second scenario (panels $2 \mathrm{~A}$ to $2 \mathrm{D}$ ) an example for rabbit 5 is shown. The intact AV node presented a unimodal histogram although both FP and SP conductions were present (panel 2B). In this specific case the mean AA interval was $100 \mathrm{~ms}$ and the most probable $\mathrm{HH}$ interval was around $200 \mathrm{~ms}$ confirming the presence of a 2:1 dominant pattern. Notice how, after ablation of the SP, the ventricular response pattern changed to a bimodal histogram although only the FP was available (2C). In this case both the $2: 1$ and the $3: 1$ atrioventricular conduction pattern were present. Similar behaviour was achieved after the ablation of the FP (2D). Here, although only the SP was available a bimodal histogram was found. This first scenario in which the ablation of the SP produced a modification from unimodal to bimodal histograms was observed during real experiments in the literature [45]

In the third scenario (panels 3A to 3D), an example for rabbit 2 is illustrated. A bimodal HH histogram was present with the intact node. In this specific case, the mean AA interval was $105 \mathrm{~ms}$ and most probable $\mathrm{HH}$ interval were around $210 \mathrm{~ms}$ and $315 \mathrm{~ms}$ confirming the presence of $2: 1$ and $3: 1 \mathrm{AV}$ conduction patterns. As it can be observed in panel $3 \mathrm{~B}$, both FP and SP conductions were present and both contribute to the short (2:1) and long (3:1) HH distributions. After the simulated ablation of the SP (panel 3C) a bimodal HH histogram remained although only FP conductions were possible. Similarly, after the FP ablation (panel 3D) a bimodal HH histogram remained although only SP conductions were available.

In the forth scenario (panels $4 \mathrm{~A}$ to $4 \mathrm{D}$ ), a second example for rabbit 2 is depicted. The only difference with the example of panels 3 was the mean atrial rate (e.g. mean AA was reduced from $105 \mathrm{~ms}$ to $100 \mathrm{~ms}$ ). In this case a bimodal HH histogram was present with the intact node also. However, in this experiment FP conductions were mainly responsible for the long $\mathrm{HH}$ distributions (3:1) whereas SP conductions were mainly responsible for the 


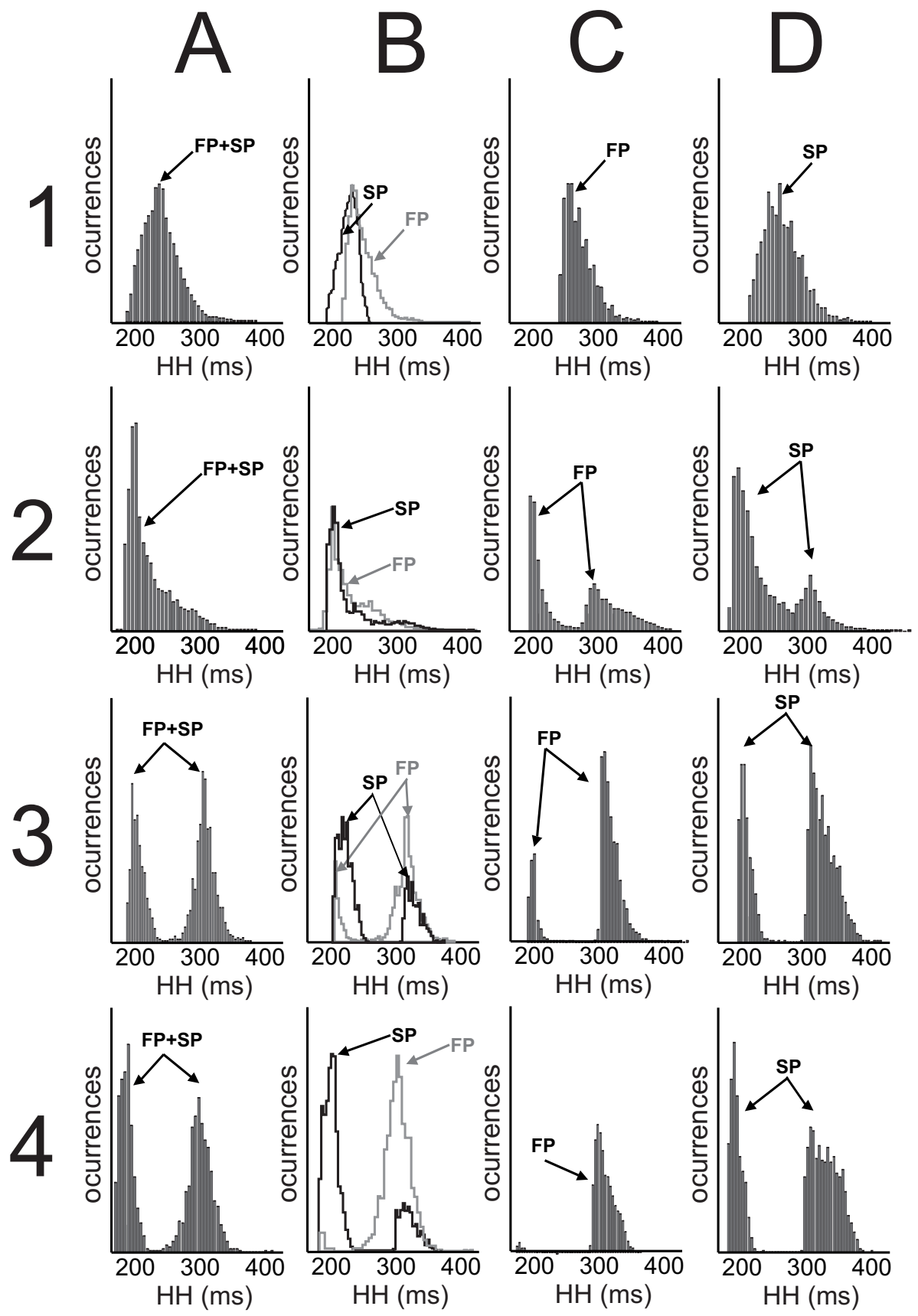

Figure 6.9: Four examples of HH interval histograms during basal conductions (A), during basal conductions but differentiating FP and SP conductions (B), after a simulated SP ablation (C) and after a simulated FP ablation (D). (See text for more details) 
short $\mathrm{HH}$ distribution (2:1) (panel 4B). In this case, the ablation of the SP (panel $4 \mathrm{C}$ ) produced an apparently unimodal $\mathrm{HH}$ histogram with only $\mathrm{FP}$ conductions with mainly a $3: 1$ conduction pattern. Notice that a little number of 2:1 conduction pattern beats were also present. Nevertheless, ablation of the FP (panel 4D) did not modify the bimodal HH histogram pattern and both short and long $\mathrm{HH}$ distributions were present although only SP conductions were present.

\subsection{Discussion and Conclusion}

\subsubsection{Major Findings}

In this study we have developed and presented a novel functional model of the AV node that includes the dual pathway AV node physiology based on experimental data. The inclusion of fast pathway (FP) and slow pathway (SP) conduction properties not only provided an excellent fit to the experimental data base, but also helped to elucidate complex and still poorly understood peculiarities of conduction through the AV node during arrhythmias.

The model has been applied to explain how the interaction between FP and SP propagation may be responsible for the development of Wenckebach rhythms (irregular periodic ventricular cycles) during regular atrial activation. Specifically, we have found that Wenckebach periodicity develops in a specific range of atrial coupling intervals and that consecutive atrial beats are conducted via either FP or SP. While Wenckebach periodicity could develop theoretically in a single-pathway model, it is the specific interaction between the two pathways in the intact heart that produces rich variability of Wenckebach patterns. These findings can help to explain some of the paradigmatic mechanisms underlying the atrioventricular conduction [61, 141, 142, 143].

In addition, the presented model has allowed an easy modification of either FP or SP in the same heart thus permitting evaluation of the effects of clinical ablations used as therapeutic tools for control of ventricular rate during atrial fibrillation (AF). Unlike live-models (such as the rabbit heart) in which only one specific destructive procedure could be performed, the mathematical model allows unrestricted "ablation" of the pathways in any order, as well as subsequent testing with wide range of pacing protocols. Thus we have illustrated the effects of $\mathrm{FP}$ and $\mathrm{SP}$ ablations during irregular atrial rates (such as AF), and demonstrated that only ablation of the SP produced a significant slowing of ventricular rate.

Finally, the role of dual AV nodal physiology in the ventricular response pattern has been evaluated. It has been demonstrated that the presence of bimodal HH interval histograms should not be used as an unequivocal index for the presence of two active pathways. It has been shown that a unimodal $\mathrm{HH}$ histogram does not necessarily imply the presence of a single pathway both FP 
and SP can produce this ventricular response pattern.

\subsubsection{Comparison with Previous AV Node Models}

Several functional models have been presented in the literature in order to calculate the AV nodal conduction time of an atrial activation as a function of preceding atrial coupling intervals $\mathrm{AA}$ and conduction times $\mathrm{AH}$ $[76,67,64,65,74,140,73,69,70]$. The model presented here can be considered an evolution of previous models. However, unlike previous models that treated the AV node as single conduit, the new model includes properties of dual AV nodal physiology based on experimental data. Dual-pathway electrophysiology was initially introduced in order to explain the AV nodal reentrant tachycardia (AVNRT) [152]. It has been later established that dual pathways are a fundamental property that governs atrio-ventricular conduction even in absence of arrhythmias [12]. Nevertheless, an anatomical model in which anterograde propagation from atria through the AVN coexists with potential retrograde propagation through different pathway has become widely used in the clinic and was validated by the introduction of successful ablation procedures to cure AVNRT by ablation of the SP, or to control ventricular rate during $\mathrm{AF}$ by modification of the pathways. Despite of all this, the precise mechanisms governing the interaction between wavefronts within the AV node remains insufficiently understood.

Our mathematical model offers new tools for deeper insight into the mechanisms of AV nodal conduction. The model is based on simple assumption that the bundle of His is activated always via the pathway that has shorter conduction time for a particular atrial beat. Conduction times are calculated based on the current atrial prematurity, as well as on the conduction of the immediately preceding beat. This information is obtained during routine clinical or experimental interrogation of AV node conduction by application of standard programmed pacing protocols.

Our model not only calculates conduction times for each of the pathways, but also permits determination of the dominant pathway for each atrial beat. The secondary pathway is considered blocked. The latter condition can develop according to two different mechanisms (see Fig. 6.2). First, due to excessive atrial prematurity cellular components within the pathway have not recovered from previous excitation and remain in refractoriness. In this case the wavefront propagating via the secondary pathway reaches inexcitable barrier and stops. Second, a wavefront may propagate successfully along the pathway but slower than the wavefront in the dominant pathway. The latter not only reaches the bundle of His, but in addition invades retrogradely the excitable secondary pathway and collides with the wavefront there. The collision results in annihilation and block within the secondary pathway [154].

Our model calculates the responses of each pathway based on its inherent conduction properties derived from the standard pacing protocols during initial 
testing, and makes final decision about which pathway, SP or FP, would be dominant or secondary. We took advantage of recently discovered powerful marker of dual pathway AV nodal conduction, the His alternans [44, 45, 46]. By monitoring the amplitude of the His electrogram spikes in a real physiological experiment one can reliably determine the dominant pathway for each conducted beat. During the development phase, our model's constants were calculated to achieve a desired fit with the experimental database for each individual heart. During the testing phase the model responded very similar to the real heart in conditions of complex and/or random interrogation protocols that resulted in arrhythmias such as Wenckebach periodicity and atrial fibrillation. Finally, the model was used to predict the outcomes of surgical AV nodal modifications involving ablation of either the SP or FP.

The model described in this study can be used to better understand and predict the mechanisms governing alternative participation of FP and SP wavefronts in the filtering task of the AV node during various arrhythmias, and could be used to assist in the selection of most optimal clinical ablation procedure for AV nodal modification in a given patient. For example, it has been suggested that ablation of the SP or partial damage of the compact AVN might be a useful treatment for ventricular rate control during AF [144, 145]. Our mathematical model shows how the effectiveness of the SP ablation depends on the percentage of SP conductions observed during AF, since according to the model higher success should be expected in subjects with predominant SP conduction.

Our model is based on exponential mathematical equations based on empirical data obtained in real heart preparations. The simplicity of the present functional model does not reflect the complexity of the anatomical structure of the AV node but allows a description of the input output relationship of $\mathrm{AV}$ nodal conduction in a form that would permit quantitative applications, including in the clinic. Future efforts to combine a realistic three-dimensional structure of the AV node [155, 92] with ionic channel modelling of the different cellular types found in the AV node [94] and their connections are likely to further our understanding of AV nodal complexity.

\subsubsection{Study Implications and Limitations}

Several limitations of the presented model should not be underestimated:

1) The model presented here includes two pathways, in accordance with our experimental data. However, multiple pathway physiology may exist as has been suggested in the literature [156]. More than two pathways could be easily introduced in the model by the construction of multiple conduction curves from experimental data.

2) In this work, both in the experimental and in the modeling parts, atrial pacing protocols were designed to apply the same input stimuli to both pathways. Even though different authors have not found significant differences 
in the AV nodal response when stimulation was applied to either the Crista Terminalis or the inter atrial septum $[116,146]$, it may be more accurate to consider independent inputs to each pathway in the AF model.

3) Complex pathway interactions, like micro-reentry or electrotonic inhibitions, cannot be detected by available markers (such as His alternans) and were not included in the model. However, they may also play an important role in the atrioventricular conduction during irregular atrial rhythms and be responsible for some errors in model predictions. Higher resolution electrical or optical mapping recordings may be needed in order to include these features in the model.

4) The anatomy of each AV junction may present important differences from one preparation to another, especially in the anatomy, cell orientations and interactions. Such variability may explain the differences found among parameters used in this functional model to accurately reproduce complex functional AV nodal responses and conduction times. Further studies of the anatomy of AV nodal junctions may help to elucidate the physiological meaning of the parameters used.

5) The effect of the autonomic tone in the modulation of AV nodal conduction has not been considered in the model. Variations in the autonomic tone may affect many of the parameters of the model considered as constant values, (i.e. AHmax or AHmin). Modeling the effect of the autonomic nervous system on the AV conduction may help in understanding the effect of vagal stimulation on the ventricular rate during atrial arrhythmias [123].

In summary, we realize that our mathematical model is derived from the rabbit heart, has limitations, and should be applied carefully to the study of human AV conduction. Data from electrophysiological studies in humans are needed in order to improve and validate the model for clinical applications. However, the presented model can help in understanding some of the intriguing AV node mechanisms and should be considered as a step forward in the studies of AV nodal conduction. 



\title{
Chapter 7
}

\section{Discussion and conclusion}

\author{
7.1 Discussion
}

7.3 Guides for future work

\subsection{Conclusion}

In this chapter a global conclusion of the results given in this dissertation is presented. This chapter has been structured in three sections. First, the main findings of this thesis are summarized and previous works relevant to this topic are discussed in light of our results. Limitations of the methods presented in the previous sections are highlighted. Secondly, the main conclusions of this work are explicitly presented and it is summarized how they satisfy the objectives previously established. Finally, a third section is dedicated to future research lines.

\subsection{Discussion}

During the development of the present thesis different characteristics of the $\mathrm{AV}$ conduction have been analyzed by using recordings from different species and with different techniques. We started analyzing one of the most intriguing behaviors of the ventricular response patterns during AF; when constructing RR-interval histograms obtained from Holter recordings with persistent AF, uni-, bi-, or multimodal RR distribution patterns can be found. In fact, more than $50 \%$ of patients during AF presented more than one RR distribution. Despite the importance of these phenomena in rate-controlling therapies for the $\mathrm{AF}$, mechanisms responsible of this observation remain controversial. In 1950, Söderström postulated that populations of RR conductions should be multiples 
of the refractory periods of the AV node [107]. It was a reasonable hypothesis, since AV nodal refractory period is modified by the vagal tone [112]. Effect of the vagal tone on the ERP should be gradual, however when we analyzed the ventricular response pattern from Holter ECGs we noted that the variation in the mean RR interval was not associated with a gradual modification of the position of predominant RR intervals. Rather than this, we noticed that the reduction of the mean $\mathrm{RR}$ during the night was caused by an increase in the number of occurrences of long RR interval populations and a decrease in the number of occurrences of short RR interval populations, whereas the position of populations did not change.

In 1956, Moe et al. presented evidence of the existence of an AV node dual physiology [23]. It was demonstrated that the so-called slow-pathway has a shorter refractory period but lower conduction velocity than the so-called fastpathway. The concept of two pathways with separated conduction properties was used later by Olsson et al. [97, 40, 95] for suggesting that predominant $\mathrm{RR}$ intervals during $\mathrm{AF}$ are caused by conduction through different $\mathrm{AV}$ node pathways. However, even when the slow pathway presents a slightly shorter refractory period than the fast pathway, both refractory periods range from 250 to $350 \mathrm{~ms}$ [113], differences that do not exceed $100 \mathrm{~ms}$, far from the positions of the predominant RR intervals observed in our results and previous studies $[40,41]$. This analysis invalidates the hypothesis from Olsson et al.

More recently, Garrigue et al. demonstrated that not the dual AV nodal pathways per se, but the complexity of the atrial-AV nodal engagement underlie the VR patterns observed during fast atrial rates $[114,115]$. In the same way, by pacing from different sites of the atria in rabbit experiments, Chorro et al. [116] concluded that the ventricular rate during AF was not only determined by the properties of the AV node but also by the rate and irregularity of the fibrillatory process. This reaffirms the idea presented by Kirsh et al.[117], who suggested that the irregularity of the VR during AF is primarily a consequence of the irregularity inherent in the atrial activity and the role of the AV node is predominantly confined to scaling the atrial activity [117].

With all this background we aimed to demonstrate that predominant $\mathrm{RR}$ intervals during $\mathrm{AF}$ are related with the atrial fibrillatory process: we hypothesized that each one of these populations may be a multiple of the mean atrial rate.

In order to validate this hypothesis we have used two main approaches, one to confirm empirically our assumptions by using real human clinical recordings and another to validate those assumptions by using mathematical models of the atrial fibrillatory process and the atrioventricular conduction:

- By analyzing Holter ECG recordings, an automatic method for the characterization of the ventricular response during $\mathrm{AF}$ by using histographic Poincaré plots has been presented. We have introduced the Poincaré Surface plot for reducing the dispersion of RR populations, 
allowing a robust and reproducible population detection. With this novel methodology we have empirically demonstrated a relation between the atrial cycle length and the ventricular response during AF. It has been illustrated how the reduction of the ventricular rate due to changes in the autonomic tone (e.g. days vs. night) does not imply variations in the position of predominant $R R$ intervals, but rather variation in the probability of occurrence of each predominant RR intervals. Similar behavior was found when the conduction properties of the AV node were modified by $\beta$-blockers. In addition, it has been illustrated how a modification in the atrial rate due to Verapamil produces shifts in the position of the pRR consistent with modified DACL values.

- By using endocardial recordings from humans and rabbits, mathematical models of the atrial activity and the atrioventricular conduction behavior have been developed and used to functionally demonstrate the main role of the atrial fibrillatory process in the ventricular response during AF. Specifically, we propose a novel methodology for the generation of realistic AA interval series during AF. We have demonstrated that Type IV Pearson can be adjusted in order to fit probability density functions of AA series with different statistical properties with higher accuracy than other distributions such as Poisson or Gaussian distribution. The proposed methodology also includes autocorrelation characteristics, which may modify the organization of the ventricular response during AF.

In addition, a novel model of the AV node that includes the dual AV nodal physiology and the concealed conduction has been presented and validated by using experimental data from rabbit AV node preparations. By using mathematical models the fundamental role of the mean AA interval series has been illustrated; the AV conduction during AF has been demonstrated to be confined to scaling the mean atrial rate. In added, the importance of the organization of the atrial bombarding into the AV node has been studied. It has been showed how the degree of transient linking [124], simulated by means of the autocorrelation coefficient and the standard deviation, can produce important differences in the ventricular response pattern even for the same AV node. We showed that low organization can produce a wide and apparently unimodal RR interval histogram, whereas higher degrees of organization of the atrial activity produced bimodal RR interval histogram. In added, mathematical models of the AV node together with the possibility to generate realistic AA interval series simulating AF has allowed us to functionally demonstrate that the presence of bimodal RR interval histograms should not be used as an unequivocal index for the presence of active fast and slow pathways. Similarly, the presence of a unimodal $\mathrm{RR}$ interval histogram does not imply the existence of only a single 
pathway since both FP and SP can produce this ventricular response pattern. Finally, these mathematical models were also useful to elucidate some complex and still poorly understood peculiarities of conduction through the AV node during arrhythmias. The model has been applied to explain how the interaction between FP and SP propagation may be responsible for the development of atypical Wenckebach rhythms (irregular periodic ventricular cycles during regular atrial activation). In addition, the presented model has allowed an easy modification of either FP or SP in the same heart thus permitting evaluation of the effects of clinical ablations used as therapeutic tools for control of the ventricular rate during $\mathrm{AF}$.

\subsubsection{Limitations}

Two main limitation of this work need to be considered:

1 The empirical demonstration of the relation between the atrial fibrillatory process and predominant $\mathrm{RR}$ intervals was based in indirect measurements from non-invasive recordings. Holter ECG signals are the only clinical signals that allow long term evaluation and consequently a detailed analysis of the effects of the autonomic tone or rate control treatments in the atrial and ventricular response during AF. However, these non-invasive recordings only allow indirect measurements of the atrial bombarding into the AV node. For a more precise demonstration of the presented hypothesis, an intracardiac mapping of the triangle of Koch and the His region may be needed. However, clinical techniques that allow this kind of recordings are not available. This has been the main reason why mathematical models have been used to demonstrate our proposed hypothesis.

2 The functional illustration of the mechanisms involving the atrioventricular conduction during atrial fibrillation was developed by using a mathematical model of the atrioventricular conduction based in rabbit experiments. This may be considered as an important limitation for the extrapolation of present results to the atrioventricular conduction in humans. In fact, as shown in chapter 4 and [109], preferential RR distributions in humans are usually the 3rd, 4th or 5th multiple of the mean atrial rate. In rabbits the most frequent $\mathrm{RR}$ interval and the effective refractory period of the AV node are shorter than in humans, consequently in rabbits predominant RR intervals are usually the 2 nd or 3 rd multiple of the mean atrial rate. This difference may have important implications in the interaction pattern between the fast and slow pathways. A similar model to that described in chapter 6 but for a human AV node may be necessary to confirm that human AV nodes present similar mechanisms than rabbit AV nodes. 


\subsection{Conclusion}

In this thesis we have accomplished all the objectives that were established. A brief response to each objective stated in section 1 is detailed in this section.

\section{Objective:}

- Development of a methodology for the characterization of the ventricular response during atrial fibrillation.

Response: A novel method, the Poincaré Surface Profile, has been introduced for reducing the dispersion of $\mathrm{RR}$ populations, allowing an accurate and reproducible $\mathrm{RR}$ population detection. The Poincaré Surface Profile method is a robust technique that allows automatic detection of preferential AV nodal conductions. Consequently, it may play a relevant role for a deeper understanding of $\mathrm{AF}$ mechanisms and for quantifying AF patterns and properties.

\section{Objective:}

- Evaluation of the role of the atrial fibrillatory activity in the ventricular response during $A F$.

Response: In order to achieve this objective two approaches have been applied: 1) the relation between predominant $\mathrm{RR}$ and multiples of the dominant atrial cycle length measured from Holter ECG signals during AF suggests that more probable RR intervals are caused by different conduction ratios of the atrial rate. 2) A novel mathematical model of the AV node, together with generated synthetic AA interval series, demonstrated the relation between mean values of AA interval series and position of predominant RR intervals.

\section{Objective:}

- Evaluation of the role of the dual AV nodal physiology in the ventricular response during $A F$.

Response: The mathematical model of the AV node, together with the possibility to generate realistic AA interval series simulating AF, has allowed us to demonstrate all different scenarios of interaction between both fast and slow atrioventricular pathways during AF. It has been shown how the existence of different predominant $R R$ interval is mainly related with the organization of the atrial bombarding into the AV node rather than in the presence of one or two atrioventricular pathways. 


\subsection{Guides for future work}

The present thesis can help in understanding some of the intriguing AV node mechanisms and should be considered as a step forward in the studies of AV nodal conduction. Our experimental and mathematical models simulations have demonstrated the important role that AA interval series characteristics play in the ventricular response during AF. In fact, conclusions of the present research may modify the way in which clinician plan rate control strategies or at least may indicate that clinical trials of rate control strategies should evaluate the specific characteristics of the fibrillatory process as a discriminatory parameter. However, several questions remain not answered after the developed of the present thesis. Some intriguing results presented here open new questions that future works may need to address.

As previously stated, mathematical models presented in this study have been based in experimental recordings from rabbits preparations. Recently the His electrogram alternance phenomenon was demonstrated in an explanted human heart [93]. This, together with new improvements on intracardiac recording equipment by means of novel electroanatomical navigation systems (i.e. CARTO or EnSite systems) will allow in the near future clinical recordings in the region of the His bundle that present the His electrogram alternance. This opens possibility for the development of mathematical models similar to that presented in this dissertation but based on human recordings. In fact, since the time used to perform recording protocols needed to adjust the model may not be longer than 10-15 minutes, a mathematical model of the AV node of each specific patient could be developed and used to select the most appropriate treatment.

Even before the development of a specific model of the AV node adapted to humans, the presented model could be used to quantify the effect of each modulating factor on each pathway and also intact AV nodes. A detailed analysis of the dependency of each parameter with other modulating factors (i.e. time after thoracotomy, rate control drugs, etc.) may be of great interest. Also, an analysis of the relation between the histological anatomy of each AV node preparation and the demonstrated differences in some parameters of the model would be a relevant study. Notice that the anatomy of each AV junction may present important differences from one preparation to another, especially in cells orientation and connexion,

In addition, the principles incorporated in our model could be used for testing and improving other more sophisticated models like the recently presented by both Efimov's group and Boyett's group [157, 91, 92, 94]. Without any kind of doubt these detailed 3D morphological reconstructions of the AV node together with detailed mathematical models of the electrophysiological behaviour of AV node cells will be useful to finally unmask some of the most intriguing characteristics of the AV node.

Although with the current technology the amount of work required for a $3 \mathrm{D}$ histological reconstruction makes unfeasible to construct an specific model 
for each individual AV node preparation, which may allow inter-individual comparisons as those developed in the present work, in the near future novel image techniques will revolutionize the way in which mathematical models are developed nowadays. In added, although today, realistic 3D model at the level of ion channels may need hours or days to simulate several seconds in some of the most powerful computers, in the near future new computer technologies will drastically reduce that computational time. All these novel techniques will lead to great advances in electrophysiology knowledge. 



\section{Chapter 8}

\section{Contributions}

As it occurs in most research works, this dissertation can not be regarded as an isolated item. On the contrary, it has been developed within the framework of current research projects, and having established close contacts with other research groups and institutions. In addition, the most important advances achieved in this thesis have been published in top level conferences and journals with high impact factor indices.

\subsection{Publications}

This thesis is supported by the publication of part of its content in three articles already published in international journals and a fourth article under the review process of an international journals at the time of the defense of the thesis, all of them listed in the Scientific Citation Index. In added, part of the work was published as a book chapter and presented in diverse international conferences.

\subsubsection{Main Contributions of This Thesis}

- Poincaré Surface Profiles of RR intervals. A novel noninvasive method for the evaluation of preferential AV nodal conduction during atrial fibrillation [109], which appeared in IEEE Transactions on Biomedical Engineering (Impact Factor: 2.154, Ranking 22/59 in category Biomedical Engineering). In this article, a new method for the evaluation of the ventricular response during atrial fibrillation is proposed.

- Role of the Atrial Rate as a Factor Modulating Ventricular Response during Atrial Fibrillation [121], which appeared in Pace-Pacing and Clinical Electrophysiology (Impact Factor: 1.578, Ranking 36/59 in category Biomedical Engineering). In this article we 
evaluated the relation between the mean atrial rate and the position of preferential RR interval distributions. For the first time a correlation between the atrial activity and the ventricular activity during AF was demonstrated.

- Realistic Generation of Atrial to Atrial Interval during Atrial Fibrillation [158], which is under review. In this work we propose a novel methodology for the generation of realistic AA interval series during AF. These synthetic AA interval series were used to evaluate the role of the atrial fibrillatory process in the ventricular response by using AV node mathematical models.

- Functional Mathematical Model of Dual Pathway AV nodal Conduction [139], American Journal of Physiology-Heart and Circulatory Physiology Impact Factor: 3.712, Ranking 17/95 in category Cardiac and Cardiovascular Systems). In this study we have developed and presented a novel functional model of the AV node that includes the dual pathway AV node physiology based on experimental data. This model may useful to understand the atrioventricular conduction during supraventricular arrhythmias and specially during Atrial Fibrillation.

\subsubsection{Contributions Related to This Thesis}

- Noninvasive assessment of the complexity and stationarity of the atrial wavefront patterns during atrial fibrillation [159], which appeared in IEEE Transactions on Biomedical Engineering (Impact Factor: 2.154, Ranking 22/59 in category Biomedical Engineering). In this work a novel automated approach to quantitatively evaluate the degree of spatio-temporal organization in the atrial activity during atrial fibrillation is presented.

- Non-invasive mapping of atrial fibrillation [160], which appeared in Journal of Cardiovascular Electrophysiology (Impact Factor: 3.703, Ranking 19/95 in category Cardiac and Cardiovascular systems). This publication describes the techniques used for constructing body surface potential maps during atrial fibrillation and the propagation patterns that we have observed in humans.

- Limitations of Dower's inverse transform for the study of atrial loops during atrial fibrillation [161], which appeared in Pacing and Clinical Electrophysiology (Impact Factor: 1.578, Ranking 36/59 in category Biomedical Engineering). In this manuscript we evaluated the performance of Dower's inverse transform and for the derivation of orthogonal leads during atrial fibrillation. Other P-wave optimized and QRS-optimized transforms were also evaluated. 
- Evaluation of lead selection methods for optimal reconstruction of body surface potentials [162], which appeared in Journal of Electrocardiology (Impact Factor: 1.077, Ranking 69/95 in category Cardiac and Cardiovascular systems). This publication evaluates two different methods proposed in the literature for the determination of optimum lead sets departing from Body Surface Potential Mapping recordings.

- How many leads are necessary for a reliable reconstruction of surface potentials during atrial fibrillation? [163], which appeared in IEEE Transactions on Information Technology in Biomedicine (Impact Factor: 1.694, ranking 27/95 in category Computer Science, Information Systems). This paper evaluated the minimum number of electrodes which allow an accurate reconstruction of body surface potentials during atrial fibrillation.

- Improving The Diagnosis Of Bundle Branch Block By The Analysis Of Body Surface Potential Maps [164], which appeared in Journal of Electrocardiology(Impact Factor: 1.077, Ranking 69/95 in category Cardiac and Cardiovascular systems). This publication describes the possible use of Body Surface Potential Mapping for an automatic diagnosis of bundle branch block.

Finally, research works related to this thesis have been presented in Spanish and international conferences in the fields of biomedical engineering, signal processing and cardiology, with a total of 40 contributions.

\subsection{Framework of the Dissertation}

This dissertation has been carried out within the Bioengineering group at the Institute for the Applications of Advanced Information and Communication Technologies (Bio-ITACA) at Universitat Politècnica de València. The researchers in the Bioengineering area, leaded by Dr. José Millet, have contrasted expertise in the analysis of cardiac arrhythmias from the ECG, such as ventricular fibrillation, ventricular tachycardia, etc. Research at (Bio-ITACA) has been devoted during the last years to the analysis of electrical activity of the heart and specially the atrial fibrillatory signals. The research at Bio-ITACA has focused in the study of non-invasive recordings such as standard-ECG, Holter ECGs or Body Surface Potential Mapping recordings. More recently, these tools are been validated by means of the analysis of intracardiac recordings and mathematical models such as those presented in this thesis. 


\subsubsection{Research Projects}

This work has been developed within the framework of several research projects. The interest and relevance of the research activity carried out in the Bio-ITACA group has been acknowledged by public administrations, which have contributed with important economical support to the following research projects:

- Ministerio de Ciencia e Innovación.

Desarrollo de tecnicas avanzadas de analisis y caracterizacin de mapas de propagacion para la ayuda al diagnostico electrocardiografico.

From Jan. 1st, 2010 until Jan. 1st, 2013.

- Ministerio de Educación.

Sistema integral de analisis de la señal electrica cardiaca para la evaluacin de arrimias supraventriculares.

From Jan. 1st, 2009 until Dec. 1st, 2010.

- Ministerio de Educación.

Exploración de nuevas técnicas de procesado y analisis de la fibrilación auricular. Aportación de los sistemas multi-derivación.

From Oct. 15th, 2005 until Jan. 1st, 2009.

- Generalitat Valenciana. Conselleria de Educacio.

PROMAPCOR: Desarrollo de tecnicas avanzadas de analisis y caracterizacin de mapas de propagacion para la ayuda al diagnostico electrocardiografico

From Jan. 1st, 2010 until Jan. 1st, 2011

- Generalitat Valenciana. Conselleria de Sanitat.

Valoracin del efecto de la ablacion sobre la distrubicion de frecuencias dominantes mediante cartografia electrica no invasiva de alta densidad de la fibrilacion auricular

From Jan. 1st, 2010 until Dec. 31st, 2010

- Generalitat Valenciana. Conselleria de Sanitat.

Estudio y caracterizacion del flutter auricular mediante capas electrocardiograficos de superficie

From Jan. 1st, 2009 until Dec. 31st, 2009

- Sociedad Española de Cardiologia

Cartografia no invasiva de alta densidad de fibrilacion auricular. Analisis de correlacion con registros

From Jan. 1st, 2009 until Dec. 31st, 2010 
- Generalitat Valenciana. Conselleria de Educacio.

Sistema integral de analisis de la señal cardiaca para la evaluacion de arritmias supraventriculares

From Jan. 1st, 2009 until Jan. 1st, 2010

- UPV. Vicerrectorado de Investigación, Desarrollo e Innovación.

Genesis del electrocardiograma de superficie en el sindromde Brugada.

From Dec. 12th, 2009 until Jan. 1st, 2011.

- UPV. Vicerrectorado de Investigación, Desarrollo e Innovación.

Extraccion de la actividad auricular en registros de mapeo electrocardiografico no invasivo de alta densidad de la fibrilacion auricular.

From Dec. 15th, 2009 until Jan. 15th, 2010.

- UPV. Vicerrectorado de Investigación, Desarrollo e Innovación.

Analisis de la onda-P del electrocardiograma para la caracterizacin de la conduccion auricular en pacientes con riesgo de sufrir episiodeos de fibrilacion auricular.

From Dec. 4th, 2007 until Dec. 4th, 2009.

\subsubsection{International Research Stays}

During the development of this thesis AM Climent has successfully completed several research stays:

- Universitats Klinikum, Magdeburg, Germany, 6 months research stay within the group leaded by Dr. Andreas Bollmann. During this stay, a joint work presented in this thesis was developed [109].

- Politecnico de Milano, Milan, Italy, 3 months under the supervision of Dr. Luca Mainardi. During this stay, a book chapter about the ventricular response during atrial fibrillation was prepared [51].

- Leipzig Heart Center, Leipzig University, Germany), 3 months research stay within the group leaded by Dr. Andreas Bollmann. During this stay, a joint work presented in this thesis was developed[121].

- Cleveland Clinic, Cleveland, Ohio, United States of America, 1 month under the supervision of Dr. Todor Mazgalev. During this stay, a joint work presented in this thesis was developed [139].

- Michigan University, Ann Arbor, Michigan, United States of America, 2 months under the supervision of Dr. Omer Berenfeld. During this stay, a joint project for the analysis of animal recordings and mathematical simulations during atrial fibrillation was started. 


\subsubsection{Collaborations}

Within the general framework of this thesis, the contacts with the following research groups and institutions have been established and reinforced:

University of Madgeburg and Leipzig University, Germany Dr. Andreas Bollmann is currently one of the most active cardiologists in AF research. After carrying out a 9-month stage within his research group, the author is further collaborating with Dr. Bollmann in a joint work whose main objective is to extend the analysis of electrocardiographic signals during AF. This collaboration has resulted in several publications and collaborations $[57,165,109,121,161,160,163]$

Cleveland Clinic, Cleveland, Ohio, United States of America The group of researchers at the Department of Molecular Cardiology in the Lerner Research Institute, leaded by Dr. Mazgalev have helped us with the development of isolated rabbit heart experiments and also with the interpretation of the results of our studies. This collaboration is resulting in interesting publications $[139,166]$.

Hospital Gregorio Marañon de Madrid, Spain. The cardiologists at the Servicio de Electrofisiologia, leaded by Dr. Felipe Atienza have helped us with the acquisition of intracardiac recordings of patients during Atrial Fibrillation and also with the interpretation of the results of our studies. This collaboration is resulting in interesting publications [158]. 


\section{Bibliography}

[1] S. Tawara, Das Reizleitungssystem des Sugetierherzens. Eine AnatomischHistologische Studie ber das Atrioventrikularbndel und die Purkinjeschen Fden. Jena: Gustav Fischer, 1906.

[2] S. Tawara, The Conduction System of the Mammalian Heart. An Anatomico-histological Study of the Atrioventricular Bundle and the Purkinje Fibers. Translated by $K$ Suma and $M$ Shimada. London: Imperial College Press, 2000.

[3] Y. H. Zhang and T. N. Mazgalev, "Ventricular rate control during atrial fibrillation and AV node modifications: past, present, and future," PacePacing and Clinical Electrophysiology, vol. 27, no. 3, pp. 382-393 2004.

[4] V. Fuster, L. E. Ryden, D. S. Cannom, H. J. Crijns, A. B. Curtis, K. A. Ellenbogen, J. L. Halperin, J. Y. L. Heuzey, G. N. Kay, J. E. Lowe, S. B. Olsson, E. N. Prystowsky, J. L. Tamargo, and S. Wann, "ACC/AHA/ESC 2006 guidelines for the management of patients with atrial fibrillation," Europace, vol. 8, no. 9, pp. 651-745 2006.

[5] R. J. Shelton, "The AFFIRM study: approaches to control rate in atrial fibrillation," Journal of the American College of Cardiology, vol. 44, no. 12, pp. 2418-2419 2004.

[6] J. Malmivuo and R. Plonsey, Bioelectromagnetism. Principles and applications of bioelectric and biomagnetic fields. New York: Oxford University Press, 1995.

[7] L. S. Lilly, Pathophysiology of heart disease. A collaborative study of medical students and faculty., 3rd ed., L. S. Lilly, Ed. Baltimore, Maryland, USA: Lippincott Williams and Wilkins, 2003.

[8] D. U. Silverthorn, Human Physiology. An integrated approach, 3rd ed. San Francisco, California, USA: Benjamin Cummings, Pearson education, 2004. 
[9] M. A. Mcguire, J. M. T. Debakker, J. T. Vermeulen, T. Opthof, A. E. Becker, and M. J. Janse, "Origin and significance of double potentials near the atrioventricular node - correlation of extracellular potentials, intracellular-potentials, and histology," Circulation, vol. 89, no. 5, pp. 2351-2360 1994.

[10] M. A. McGuire, J. M. T. deBakker, J. T. Vermeulen, A. F. M. Moorman, P. Loh, B. Thibault, J. L. M. Vermeulen, A. E. Becker, and M. J. Janse, "Atrioventricular junctional tissue - discrepancy between histological and electrophysiological characteristics," Circulation, vol. 94, no. 3, pp. 5715771996 .

[11] R. H. Anderson, S. Y. Ho, and A. E. Becker, "Anatomy of the human atrioventricular junctions revisited," Anatomical Record, vol. 260, no. 1, pp. 81-91 2000.

[12] T. N. Mazgalev, S. Y. Ho, and R. H. Anderson, "Anatomicelectrophysiological correlations concerning the pathways for atrioventricular conduction," Circulation, vol. 103, no. 1524-4539; 22, pp. 2660-2667 2001.

[13] T. N. Mazgalev and Y. Zhang, "The dual pathway electrophysiology of the atrioventricular conduction. A new look at an old phenomenon," Minerva Cardioangiologica, vol. 51, pp. 1-14 2003.

[14] D. Sanchez-Quintana and S. Y. Ho, "Anatomy of cardiac nodes and atrioventricular specialized conduction system," Revista espanola de cardiologia, vol. 56, no. 11, pp. 1085-1092 2003.

[15] T. Kurian, C. Ambrosi, W. Hucker, V. Federov, and I. Efimov, "Anatomy and electrophysiology of the human AV node," Pace-Pacing and Clinical Electrophysiology, vol. 33, no. 6, pp. 754-762 2010.

[16] T. Mazgalev and P. J. Tchou, Atrial-AV Nodal Electrophysiology: A View from the Millennium. Blackwell Publishing, 2000.

[17] W. Koch, "Weiter mitteilungen uber den sinusknoten der herzens." Verhandlungen der Deutshen Pathologischen Gesellschaft, vol. 13, no. 85 1909.

[18] M. Antz, B. J. Scherlag, K. Otomo, J. Pitha, C. Tondo, E. Patterson, W. M. Jackman, and R. Lazzara, "Evidence for multiple atrio-AV nodal inputs in the normal dog heart," Journal of Cardiovascular Electrophysiology, vol. 9, no. 4, pp. 395-408 1998.

[19] K. Kawamura and T. N. James, "Comparative ultrastructure of cellular junctions in working myocardium and conduction system under normal 
and pathologic conditions," Journal of Molecular and Cellular Cardiology, vol. 3, no. 1, pp. 31-40 1971.

[20] A. P. Decarvalho and D. F. Dealmeida, "Spread of activity through the atrioventricular node," Circulation research, vol. 8, no. 4, pp. 801-809 1960 .

[21] J. Billette, "Atrioventricular nodal activation during periodic premature stimulation of the atrium," American Journal of Cardiology, vol. 252, no. 0002-9513; 1, pp. H163-H177 1987.

[22] F. L. Meijler and M. J. Janse, "Morphology and electrophysiology of the mammalian atrioventricular node," Physiological Reviews, vol. 68, no. 2, pp. 608-647 1988.

[23] G. K. Moe, J. B. Preston, and H. Burlington, "Physiologic evidence for a dual A-V transmission system," Circulation Research, vol. 4, pp. 357-375 1956 .

[24] R. M. Schuilenburg and D. Durrer, "Atrial echo beats in the human heart elicited by induced atrial premature beats," Circulation, vol. 37, no. 5, pp. 680-693 1968.

[25] C. Mendez and G. K. Moe, "Demonstration of a dual A-V nodal conduction system in the isolated rabbit heart," Circulation Research, vol. 19, no. 2, pp. 378-393 1966.

[26] S. Bharati and M. Lev, Anatomy of the normal conduction system, diseas-related changes, and their relationship to arrhythmogenesis, ser. Cardiac arrhythmias:mechanisms, diagnosis, and management. Baltimor: Williams and Wilkins, 1995.

[27] A. Keith, "An autobiography," New York: Philosophical Library, pp. 2542591950.

[28] R. H. Anderson and S. Y. Ho, "The morphology of the atrial approaches to the AV node," Journal of Cardiovascular Electrophysiology, vol. 9, no. 3, pp. 336-337 1998.

[29] S. Nattel, "New ideas about atrial fibrillation 50 years on," Nature, vol. 415, no. 6868, pp. 219-216 2002.

[30] M. Haissaguerre, P. Jais, D. C. Shah, A. Takahashi, M. Hocini, G. Quiniou, S. Garrigue, A. Le Mouroux, P. Le Metayer, and J. Clementy, "Spontaneous initiation of atrial fibrillation by ectopic beats originating in the pulmonary veins," New England Journal of Medicine, vol. 339, no. 10, pp. 659-666 1998. 
[31] J. Jalife, O. Berenfeld, and M. Mansour, "Mother rotors and fibrillatory conduction: a mechanism of atrial fibrillation," Cardiovascular Research, vol. 54, no. 2, pp. 204-216 2002 .

[32] M. Allessie, J. Ausma, and U. Schotten, "Electrical, contractile and structural remodeling during atrial fibrillation," Cardiovascular Research, vol. 54, no. 2, pp. 230-246 2002 .

[33] D. G. Wyse and B. J. Gersh, "Atrial fibrillation: a perspective. Thinking inside and outside the box," Circulation, vol. 109, pp. 3089-3095 2004.

[34] W. A. Wattigney, G. A. Mensah, and J. B. Croft, "Increasing trends in hospitalization for atrial fibrillation in the united states, 1985 through 1999," Circulation, vol. 108, pp. 711-716 2003.

[35] R. Brugada, T. Tapscott, G. Z. Czernuszewicz, A. J. Marian, A. Iglesias, L. Mont, J. Brugada, J. Girona, A. Domingo, L. L. Bachinski, and R. Roberts, "Identification of a genetic locus for familial atrial fibrillation," New England Journal of Medicine, vol. 336, no. 13, pp. 905-911 1997.

[36] F. Atienza, J. Almendral, J. Jalife, S. Zlochiver, R. Ploutz-Snyder, E. Torrecilla, A. Arenal, J. Kalifa, F. Fernandez-Aviles, and O. Berenfeld, "Real-time dominant frequency mapping and ablation of dominant frequency sites in atrial fibrillation with left-to-right frequency gradients predicts long-term maintenance of sinus rhythm," Heart Rhythm, vol. 6, no. 1, pp. 33-40 2009.

[37] A. M. Guillinov, P. M. McCarthy, N. M. N, and A. Natale, "Contemporary surgical treatment for atrial fibrillation." Pacing and Clinical Electrophysiology, vol. 26, no. 7, pp. 1641-1644 2003.

[38] E. Prystowsky, I. Niazi, A. Curtis, D. Wilber, T. Bahnson, K. Ellenbogen, A. Dhala, D. Bloomfield, M. Gold, A. Kadish, R. Fogel, M. Gonzalez, L. Belardinelli, R. Shreeniwas, and A. Wolff, "Termination of paroxysmal supraventricular tachycardia by tecadenoson (CVT-510), a novel A1adenosine receptor agonist." Journal of the American College of Cardiology, vol. 17, no. 42, pp. 1092-1102 2003.

[39] F. Fillette, G. Fontaine, R. Frank, and Y. Grosgogeat, "The ventricular response in atrial-fibrillation - Normal conduction, consequences of conduction disorders and of preexcitation syndromes," Annales de Cardiologie et D'Angeiologie, vol. 32, no. 1, pp. 7-19 1983.

[40] S. B. Olsson, N. Cai, M. Dohnal, and K. K. Talwar, "Noninvasive support for and characterization of multiple intranodal pathways in patients with mitral-valve disease and atrial-fibrillation," European Heart Journal, vol. 7, no. 4, pp. 320-333 1986. 
[41] S. Rokas, S. Gaitanidou, S. Chatzidou, N. Agrios, and S. Stamatelopoulos, "A noninvasive method for the detection of dual atrioventricular node physiology in chronic atrial fibrillation," American Journal of Cardiology, vol. 84, no. 12, pp. 1442-1445 1999.

[42] J. Tebbenjohanns, B. Schumacher, T. Korte, M. Niehaus, and D. Pfeiffer, "Bimodal RR interval distribution in chronic atrial fibrillation: Impact of dual atrioventricular nodal physiology on long-term rate control after catheter ablation of the posterior atrionodal input," Journal of Cardiovascular Electrophysiology, vol. 11, no. 7, pp. 497-503 2000.

[43] S. Rokas, S. Gaitanidou, S. Chatzidou, C. Pamboucas, D. Achtipis, and S. Stamatelopoulos, "Atrioventricular node modification in patients with chronic atrial fibrillation - Role of morphology of RR interval variation," Circulation, vol. 103, no. 24, pp. 2942-2948 2001.

[44] Y. Zhang, S. Bharati, K. A. Mowrey, S. Zhuang, P. J. Tchou, and T. N. Mazgalev, "His electrogram alternans reveal dual-wavefront inputs into and longitudinal dissociation within the bundle of His," Circulation, vol. 104, no. 7, pp. 832-838 2001.

[45] Y. Zhang, S. Bharati, K. A. Mowrey, and T. N. Mazgalev, "His electrogram alternans reveal dual atrioventricular nodal pathway conduction during atrial fibrillation: the role of slow-pathway modification," Circulation, vol. 107, no. 7, pp. 1059-1065 2003.

[46] Y. Zhang, S. Bharati, R. Sulayman, K. A. Mowrey, P. J. Tchou, and T. N. Mazgalev, "Atrioventricular nodal fast pathway modification: mechanism for lack of ventricular rate slowing in atrial fibrillation," Cardiovascular Research, vol. 61, no. 1, pp. 45-55 2004.

[47] Y. H. Zhang, K. A. Mowrey, and T. Mazgalev, "Does RR interval distribution during atrial fibrillation indicate dual pathway atrioventricular nodal electrophysiology?" Pace-Pacing and Clinical Electrophysiology, vol. 26, p. 11102003.

[48] A. C. Rankin and A. J. Workman, "Rate control in atrial fibrillation: role of atrial inputs to the AV node," Cardiovascular Research, vol. 44, no. 2, pp. 249-251 1999.

[49] A. Bollmann and F. Lombardi, "Electrocardiology of atrial fibrillation Current knowledge and future challenges," IEEE Engineering in Medicine and Biology Magazine, vol. 25, no. 6, pp. 15-23 2006.

[50] K. M. Stein, J. Waldeen, N. Lippman, and B. B. Lerman, "Ventricular response in atrial fibrillation: random or deterministic?" American Journal of Physiology-Heart and Circulatory Physiology, vol. 277, no. 2, pp. H452-H458 1999. 
[51] V. D. Corino, A. M. Climent, L. T. Mainardi, and A. Bollmann, Analysis of Ventricular Response during Atrial Fibrillation, ser. Understanding Atrial Fibrillation: The Signal Processing Contribution. Morgan and Claypool Publishers, 2008, ch. 5, pp. 105-126.

[52] M. P. van den Berg, T. V. Noord, J. Brouwer, J. Haaksma, D. J. V. Veldhuisen, H. J. G. M. Crijns, and I. C. V. Gelder, "Clustering of RR intervals predicts effective electrical cardioversion for atrial fibrillation," Journal of Cardiovascular Electrophysiology, vol. 15, no. 9, pp. 1027-1033 2004 .

[53] A. Bollmann, D. Husser, L. Mainardi, F. Lombardi, P. Langley, A. Murray, J. J. Rieta, J. Millet, S. B. Olsson, M. Stridh, and L. Sornmo, "Analysis of surface electrocardiograms in atrial fibrillation: techniques, research, and clinical applications," Europace, vol. 8, no. 11, pp. 911-926 2006.

[54] T. Anan, K. Sunagawa, H. Araki, and M. Nakamura, "Arrhythmia analysis by successive RR plotting," Journal of Electrocardiology, vol. 23, no. 3, pp. 243-248 1990.

[55] A. S. Chishaki, K. Sunagawa, K. Hayashida, M. Sugimachi, and M. Nakamura, "Identification of the rate-dependent functional refractory period of the atrioventricular node in simulated atrial-fibrillation," American Heart Journal, vol. 121, no. 3, pp. 820-826 1991.

[56] A. C. Suyama, K. Sunagawa, M. Sugimachi, T. Anan, K. Egashira, and A. Takeshita, "Differentiation between aberrant ventricular conduction and ventricular ectopy in atrial-fibrillation using RR interval scattergram," Circulation, vol. 88, no. 5, pp. 2307-2314 1993.

[57] A. M. Climent, D. Husser, V. D. Corino, L. Mainardi, J. Millet, H. Klein, and A. Bollmann, "Non-invasive assessment of atrioventricular conduction properties and their effects on ventricular response in atrial fibrillation," Computers in Cardiology 2006, vol. 33, pp. 105-108 2006.

[58] J. Hayano, S. Sakata, A. Okada, S. Mukai, and T. Fujinami, "Circadian rhythms of atrioventricular conduction properties in chronic atrial fibrillation with and without heart failure," Journal of the American College of Cardiology, vol. 31, no. 1, pp. 158-166 1998.

[59] J. Hayano, S. Ishihara, H. Fukuta, S. Sakata, S. Mukai, N. Ohte, and G. Kimura, "Circadian rhythm of atrioventricular conduction predicts long-term survival in patients with chronic atrial fibrillation," Chronobiology International, vol. 19, no. 3, pp. 633-648 2002.

[60] T. Oka, T. Nakatsu, S. Kusachi, Y. Tominaga, S. Toyonaga, H. Ohnishi, M. Nakahama, I. Komatsubara, M. Murakami, and T. Tsuji, "Doublesector Lorenz plot scattering in an R-R interval analysis of patients with 
chronic atrial fibrillation - Incidence and characteristics of vertices of the double-sector scattering," Journal of Electrocardiology, vol. 31, no. 3, pp. 227-235 1998.

[61] G. M. Decherd and A. Ruskin, "The mechanism of the Wenckebach type of A-V block," British Heart Journal, vol. 8, no. 1, pp. 6-16 1946.

[62] R. Langendorf, "Concealed A-V conduction: the effect of blocked impulses on the formation and conduction of subsequent impulses," American Heart Journal, vol. 35, pp. 542-552 1948.

[63] G. K. Moe and J. A. Abildskov, "Observations on ventricular dysrhythmia associated with atrial fibrillation in dog heart," Circulation Research, vol. 14, no. 5, pp. 447-460 1964.

[64] R. M. Heethaar, D. van der Gon JJ, and F. L. Meijler, "Mathematical model of A-V conduction in the rat heart," Cardiovascular Research, vol. 7, no. 1, pp. 105-114 1973.

[65] R. M. Heethaar, J. J. van der Gon, and F. L. Meijler, "Interpretation of some properites of A-V conduction with the help of analog simulation," European Journal of Cardiology, vol. 1, no. 1, pp. 87-93 1973.

[66] S. Teague, S. Collins, D. Wu, P. Denes, K. Rosen, and R. Arzbaecher, "A quantitative description of normal AV nodal conduction curve in man," Journal of Applied Physiology, vol. 40, no. 1, pp. 74-78 1976.

[67] R. J. Cohen and R. D. Berger, "A quantitative model for the ventricular response during atrial-fibrillation," IEEE Transactions on Biomedical Engineering, vol. 30, no. 12, pp. 769-781 1983.

[68] L. Dorveaux and A. Tonkin, "A comprehensive model describing conduction through the atrioventricular node," International Journal of Biomedical Computing, vol. 18, no. 3-4, pp. 261-271 1986.

[69] A. Shrier, H. Dubarsky, M. Rosengarten, M. R. Guevara, S. Nattel, and L. Glass, "Prediction of complex atrioventricular conduction rhythms in humans with use of the atrioventricular nodal recovery curve," Circulation, vol. 76, no. 6, pp. 1196-1205 1987.

[70] M. Talajic, D. Papadatos, C. Villemaire, L. Glass, and S. Nattel, "A unified model of atrioventricular nodal conduction predicts dynamic changes in Wenckebach periodicity," Circulation Research, vol. 68, no. 5, pp. 1280-1293 1991.

[71] W. Zeng and L. Glass, "Statistical properties of heartbeat intervals during atrial fibrillation," Physical Reviews E., vol. 54, no. 2, pp. 1779-1784 1996. 
[72] P. Jorgensen, C. Schafer, P. G. Guerra, M. Talajic, S. Nattel, and L. Glass, "A mathematical model of human atrioventricular nodal function incorporating concealed conduction," Bulletin of Mathematical Biology, vol. 64, no. 6, pp. 1083-1099 2002.

[73] L. Mangin, A. Vinet, P. Page, and L. Glass, "Effects of antiarrhythmic drug therapy on atrioventricular nodal function during atrial fibrillation in humans," Europace, vol. 7 Suppl 2, pp. 71-82 2005.

[74] J. Lian, D. Mussig, and V. Lang, "Computer modeling of ventricular rhythm during atrial fibrillation and ventricular pacing," IEEE Transactions on Biomedical Engineering, vol. 53, no. 8, pp. 1512-1520 2006.

[75] M. Simson and J. S. adn E.N. Moore, "The relationship between atrioventricular nodal refractoriness and the functional refractory period in the dog." Circulation Research, vol. 44, no. 1, pp. 121-126 1979.

[76] F. J. Chorro, R. Ruiz-Granell, E. Casadan, R. Garcia-Civera, L. Such, and V. Lopez-Merino, "Mathematical descriptions of AV nodal function curves in dogs," Pace-Pacing and Clinical Electrophysiology, vol. 11, no. 6, pp. 679-6861988.

[77] J. Lian, G. D. Clifford, D. Mussig, and V. Lang, "Open source model for generating RR intervals in atrial fibrillation and beyond," Biomedical Engineering Online, vol. 62007.

[78] J. Lian, D. Mussig, and V. Lang, "Ventricular rate smoothing for atrial fibrillation: a quantitative comparison study," Europace, vol. 9, no. 7, pp. 506-5132007.

[79] J. Lian, D. Mussig, and V. Lang, "On the role of ventricular conduction time in rate stabilization for atrial fibrillation," Europace, vol. 9, no. 5, pp. 289-293 2007.

[80] A. Hodkin and A. Huxley, "A quantitative description of membrane current and its application to conduction and excitation in nerve," Journal of Physiology, vol. 117, pp. 500-544 1952.

[81] H. Landahl and D. Griffeath, "A mathematical model for fist degree block and the Wenckebach phenomenon," Bulletin of Mathematical Biophysics, vol. 33, pp. 27-38 1971.

[82] J. Keener, "On cardiac arrythmias: Av conduction block," Journal of Mathematical Biology, vol. 12, no. 2, pp. 215-225 1981. 
[83] M. Delmar, L. Glass, D. C. Michaels, and J. Jalife, "Ionic basis and analytical solution of the Wenckebach phenomenon in guinea pig ventricular myocytes," Circulation Research, vol. 65, no. 3, pp. 775-788 1989.

[84] M. Delmar and J. Jalife, "Analysis of rate-dependent activation in single atrioventricular nodal cells," Annals New York Academy of Sciences, vol. 591, pp. 23-37 1990.

[85] K. Hoshino, J. Anumonwo, M. Delmar, and J. Jalife, "Wenckebach periodicity in single atrioventricular nodal cells from the rabbit heart," Circulation, vol. 82, no. 0009-7322; 6, pp. 2201-2216 1990.

[86] N. Ikeda, K. Hoshino, M. Shirataka, K. Doi, H. Miyahra, and T. Sato, "A mathematical model of atrioventricular conduction block using the exctiability recovery curve of the myocardial cell," Journal of Electrocardiology, vol. 23, pp. 96-101 1991.

[87] A. R. LeBlanc and B. Dube, "Propagation in the AV node: a model based on a simplified two-dimensional structure and a bidomain tissue representation," Medical and Biological Engineering and Computing, vol. 31, no. 0140-0118; 6, pp. 545-556 1993.

[88] Y. Liu, W. Zeng, M. Delmar, and J. Jalife, "Ionic mechanisms of electronic inhibition and concealed conduction in rabbit atrioventricular nodal myocytes," Circulation, vol. 88, no. 4, pp. 1634-1646 1993.

[89] C. T. Leffler and J. P. Saul, "Computer-model of the atrioventricular node predicts reentrant arrhythmias," Pace-Pacing and Clinical Electrophysiology, vol. 17, no. 1, pp. 113-130 1994.

[90] F. L. Meijler, J. Jalife, J. Beaumont, and D. Vaidya, "AV nodal function during atrial fibrillation: The role of electrotonic modulation of propagation," Journal of Cardiovascular Electrophysiology, vol. 7, no. 9, pp. 843-861 1996.

[91] W. J. Hucker, V. P. Nikolski, and I. R. Efimov, "Optical mapping of the atrioventricular junction," Journal of Electrocardiology, vol. 38, no. 00220736; 4, pp. 121-125 2005.

[92] J. Li, I. D. Greener, S. Inada, V. P. Nikolski, M. Yamamoto, J. C. Hancox, H. Zhang, R. Billeter, I. R. Efimov, H. Dobrzynski, and M. R. Boyett, "Computer three-dimensional reconstruction of the atrioventricular node," Circulation Research, vol. 102, no. 8, pp. 975-985 2008 . 
[93] W. J. Hucker, V. V. Fedorov, K. V. Foyil, N. Moazami, and I. R. Efimov, "Images in cardiovascular medicine. optical mapping of the human atrioventricular junction," Circulation, vol. 117, no. 1524-4539; 11, pp. 1474-1477 2008.

[94] S. Inada, J. C. Hancox, H. Zhang, and M. R. Boyett, "One-dimensional mathematical model of the atrioventricular node including atrio-nodal, nodal, and nodal-His cells," Biophysical Journal, vol. 97, no. 8, pp. 211721272009.

[95] N. Cai, M. Dohnal, and S. B. Olsson, "Methodological aspects of the use of Heart-Rate stratified RR interval histograms in the analysis of atrioventricular-conduction during atrial fibrillation," Cardiovascular Research, vol. 21, no. 6, pp. 455-462 1987.

[96] S. Gaitanidou, S. Rokas, C. Pamboucas, D. Actipis, S. Chatzidou, J. Darsinos, S. Stamatelopoulos, and S. Moulopoulos, "The RR interval distribution pattern as a predictive factor of the outcome of atrioventricular conduction modification in patients with atrial fibrillation," Journal of the American College of Cardiology, vol. 31, no. 2, pp. 333A-334A 1998.

[97] M. P. Ingemansson, J. Carlson, and S. B. Olsson, "Modification of intrinsic AV-nodal properties by magnesium in combination with glucose, insulin, and potassium (GIK) during chronic atrial fibrillation," Journal of Electrocardiology, vol. 31, no. 4, pp. 281-292 1998.

[98] J. Tebbenjohanns, B. Schumacher, D. Pfeiffer, and M. Manz, "Influence of slow pathway ablation on ventricular response during atrial-fibrillation acute and follow-up results," Circulation, vol. 90, no. 4, pp. 336-336 1994.

[99] J. Tebbenjohanns, D. Pfeiffer, B. Schumacher, W. Jung, M. Manz, and B. Luderitz, "Slowing of the ventricular rate during atrial-fibrillation by ablation of the slow pathway of AV nodal reentrant tachycardia," Journal of Cardiovascular Electrophysiology, vol. 6, no. 9, pp. 711-715 1995.

[100] P. Weismuller, C. Braunss, C. Ranke, and H. J. Trappe, "Multiple AV nodal pathways with multiple peaks in the RR interval histogram of the holter monitoring ECG during atrial fibrillation," Pace-Pacing and Clinical Electrophysiology, vol. 23, no. 11, pp. 1921-1924 2000.

[101] P. Weismuller, C. Kratz, B. Brandts, K. Kattenbeck, H. J. Trappe, and C. Ranke, "AV nodal pathways in the R-R interval histogram of the 24-hour monitoring ECG in patients with atrial fibrillation," Annals of Noninvasive Electrocardiology, vol. 6, no. 4, pp. 285-289 2001. 
[102] M. Brennan, M. Palaniswami, and P. Kamen, "Do existing measures of poincare plot geometry reflect nonlinear features of heart rate variability?" IEEE Transactions on Biomedical Engineering, vol. 48, no. 11, pp. 134213472001.

[103] A. C. Suyama, "Aberrant ventricular conduction and ventricular ectopy reply," Circulation, vol. 89, no. 6, pp. 2945-2945 1994.

[104] A. Yamada, J. Hayano, S. Sakata, A. Okada, S. Mukai, N. Ohte, and G. Kimura, "Reduced ventricular response irregularity is associated with increased mortality in patients with chronic atrial fibrillation," Circulation, vol. 102, no. 3, pp. 300-306 2000.

[105] B. K. Bootsma, A. J. Hoelen, J. Strackee, and F. L. Meijler, "Analysis of RR intervals in patients with atrial fibrillation at rest and during exercise," Circulation, vol. 41, no. 5, pp. 783-794 1970.

[106] L. Toivonen, A. Kadish, W. Kou, and F. Morady, "Determinants of the ventricular rate during atrial-fibrillation," Journal of the American College of Cardiology, vol. 16, no. 5, pp. 1194-1200 1990.

[107] N. Soderstrom, "What is the reason for the ventricular arrhythmia in cases of auricular fibrillation?" American Heart Journal, vol. 40, no. 2, pp. 212-223 1950.

[108] F. Castells, C. Mora, J. J. Rieta, D. Moratal-Perez, and J. Millet, "Estimation of atrial fibrillatory wave from single-lead atrial fibrillation electrocardiograms using principal component analysis concepts," Medical and Biological Engineering and Computing, vol. 43, no. 5, pp. 557-560 2005 .

[109] A. M. Climent, M. S. Guillem, D. Husser, F. Castells, J. Millet, and A. Bollmann, "Poincare surface profiles of RR intervals. A novel noninvasive method for the evaluation of preferential AV nodal conduction during atrial fibrillation," IEEE Transactions on Biomedical Engineering, vol. 56, no. 2, pp. 433-442 2009.

[110] F. L. Meijler and J. Jalife, AV node function during atrial fibrillation, ser. Atrial-AV Nodal Electrophysiology: A View from the Millennium. Armonk, NY: Futura Publishing Company, 2000, ch. 16, pp. 251-268.

[111] A. R. Gelzer, N. S. Moise, D. Vaidya, K. A. Wagner, and J. Jalife, "Temporal organization of atrial activity and irregular ventricular rhythm during spontaneous atrial fibrillation: an in vivo study in the horse," Journal of Cardiovascular Electrophysiology, vol. 11, no. 7, pp. 773-784 2000 . 
[112] M. Nayebpour, M. Talajic, C. Villemaire, and S. Nattel, "Vagal modulation of the rate-dependent properties of the atrioventricular node," Circulation Research, vol. 67, no. 5, pp. 1152-1166 1990.

[113] F. Hegbom, O. M. Orning, M. Heldal, and K. Gjesdal, "Effects of ablation, digitalis, and beta-blocker on dual atrioventricular nodal pathways and conduction during atrial fibrillation," Journal of Cardiovascular Electrophysiology, vol. 15, no. 10, pp. 1141-1146 2004.

[114] S. Garrigue, P. J. Tchou, and T. N. Mazgalev, "Role of the differential bombardment of atrial inputs to the atrioventricular node as a factor influencing ventricular rate during high atrial rate," Cardiovascular Research, vol. 44, no. 2, pp. 344-355 1999.

[115] S. Garrigue, K. A. Mowrey, G. Fahy, P. J. Tchou, and T. N. Mazgalev, "Atrioventricular nodal conduction during atrial fibrillation: role of atrial input modification," Circulation, vol. 99, no. 17, pp. 2323-2333 1999.

[116] F. J. Chorro, C. J. Kirchhof, J. Brugada, and M. A. Allessie, "Ventricular response during irregular atrial-pacing and atrial-fibrillation," American Journal of Physiology-Heart and Circulatory Physiology, vol. 259, no. 4, pp. H1015-H1021 1990.

[117] J. A. Kirsh, A. V. Sahakian, J. M. Baerman, and S. Swiryn, "Ventricular response to atrial fibrillation: role of atrioventricular conduction pathways," Journal of the American College of Cardiology, vol. 12, no. 5, pp. 1265-1272 1988.

[118] F. X. Roitinger, A. SippensGroenewegen, M. R. Karch, P. R. Steiner, W. S. Ellis, and M. D. Lesh, "Organized activation during atrial fibrillation in man," Journal of Cardiovascular Electrophysiology, vol. 9, no. 5, pp. 451-461 1998.

[119] Y. Zhang and T. N. Mazgalev, "Achieving regular slow rhythm during atrial fibrillation without atrioventricular nodal ablation: selective vagal stimulation plus ventricular pacing," Heart Rhythm, vol. 1, no. 4, pp. 469-475 2004.

[120] F. J. Chorro, J. Sanchis, V. Lopez-Merino, L. Such, J. A. Avellana, and V. Valentin, "Effects of atrial impulse timing on AV concealed conduction in the rabbit heart," Pace-Pacing and Clinical Electrophysiology, vol. 14, no. 0147-8389; 5, pp. 842-853 1991.

[121] A. M. Climent, M. S. Guillem, D. Husser, F. Castells, J. Millet, and A. Bollmann, "Role of the atrial rate as a factor modulating ventricular response during atrial fibrillation," Pace-Pacing and Clinical Electrophysiology, vol. 33, no. 12, pp. 1510-1517 2010. 
[122] H. A. Makse, S. Havlin, M. Schwartz, and H. E. Stanley, "Method for generating long-range correlations for large systems," Physical Review E, vol. 53, no. 5, pp. 5445-5449 1996.

[123] T. N. Mazgalev, S. Garrigue, K. A. Mowrey, Y. Yamanouchi, and P. J. Tchou, "Autonomic modification of the atrioventricular node during atrial fibrillation: role in the slowing of ventricular rate," Circulation, vol. 99, no. 21, pp. 2806-2814 1999.

[124] E. P. Gerstenfeld, A. V. Sahakian, and S. Swiryn, "Evidence for transient linking of atrial excitation during atrial fibrillation in humans," Circulation, vol. 86, no. 2, pp. 375-382 1992.

[125] A. T. Schoenwald, A. V. Sahakian, H. J. Sih, and S. Swiryn, "Further observations of "linking" of atrial excitation during clinical atrial fibrillation," Pace-Pacing and Clinical Electrophysiology, vol. 21, no. 1 Pt 1, pp. 25-34 1998.

[126] S. Chen, H. Nie, and B. Ayers-Glassey, "Lognormal sum approximation with a variant of type IV Pearson distribution," IEEE Communications Letters, vol. 12, no. 9, pp. 630-632 2008.

[127] J. Heinrich, "A guide to the pearson type IV distribution," [Online Available]: http://www.cdf.fnal.gov/ 2004.

[128] R. Kwan and C. Leung, "On the applicability of the pearson method for approximating distributions in wireless communications," IEEE Transactions on Communications, vol. 55, pp. 2065-2069 2007.

[129] H. Nie and S. H. Chen, "Lognormal sum approximation with type IV Pearson distribution," IEEE Communications Letters, vol. 11, pp. 7907922007.

[130] K. Pearson, "Contributions to the mathematical theory of evolution. II. Skew variation in homogeneous material," Philosophical Transactions of the Royal Society of London. A, vol. 186, pp. 343-414 1895.

[131] Q. T. Zhang and S. H. Song, "A systematic procedure for accurately approximating lognormal-sum distributions," IEEE Transactions on Vehicular Technology, vol. 57, no. 1, pp. 663-666 2008.

[132] L. Devroye, "On random variate generation when only moments or Fourier coefficients are known," Mathematics and Computers in Simulation, vol. 31, no. 1-2, pp. 71-89 1989.

[133] Y. Nagahara, "A method of simulating multivariate nonnormal distributions by the pearson distribution system and estimation," Computational Statistics and Data Analysis, vol. 47, no. 1, pp. 1-29 2004. 
[134] L. Faes, G. Nollo, R. Antolini, F. Gaita, and F. Ravelli, "A method for quantifying atrial fibrillation organization based on wave-morphology similarity," IEEE Transactions on Biomedical Engineering, vol. 49, no. 12, pp. 1504-1513 2002 .

[135] T. M. T. Cover, Elements of information Theory. New York: Wiley, 1991.

[136] F. M. Izrailev, A. A. Krokhin, N. M. Makarov, and O. V. Usatenko, "Generation of correlated binary sequences from white noise," Physical Review E, vol. 762007.

[137] C. J. Meurling, J. E. P. Waktare, F. Holmqvist, A. Hedman, A. J. Camm, S. B. Olsson, and M. Malik, "Diurnal variations of the dominant cycle length of chronic atrial fibrillation," American Journal of Physiology-Heart and Circulatory Physiology, vol. 280, no. 1, pp. H401-H406 2001.

[138] F. Sandberg, A. Bollmann, D. Husser, M. Stridh, and L. Sornmo, "Circadian variation in dominant atrial fibrillation frequency in persistent atrial fibrillation," Physiological Measurement, vol. 31, no. 4, pp. 531-542 2010 .

[139] A. M. Climent, M. S. Guillem, Y. Zhang, J. Millet, and T. Mazgalev, "Functional mathematical model of dual pathway AV nodal conduction," American Journal of Physiology-Heart and Circulatory Physiology, vol. 300, no. 4, pp. H1393-401 2011.

[140] V. Lopez-Merino, L. D. Insa, J. A. Ferrero, S. Botella, R. Llopis, J. Merino, S. Morell, and J. Chorro, "Function of atrioventricular node conduction: hyperbolic model," Revista Española de Fisiología, vol. 37, no. 2, pp. 2212301981.

[141] M. Duytschaever, C. Dierickx, and R. Tavernier, "Variable atrioventricular block during atrial flutter: what is the mechanism?" Journal of Cardiovascular Electrophysiology, vol. 13, no. 9, pp. 950-951 2002.

[142] P. Maury, F. Raczka, C. Piot, and J. M. Davy, "QRS and cycle length alternans during paroxysmal supraventricular tachycardia: what is the mechanism?" Journal of Cardiovascular Electrophysiology, vol. 13, no. 1, pp. 92-93 2002 .

[143] S. Richter, A. Berruezo, L. Mont, T. Boussy, A. Sarkozy, P. Brugada, and J. Brugada, "Pseudo-atrial fibrillation, rare manifestation of multiple anterograde atrioventricular nodal pathways," American Journal of Cardiology, vol. 100, no. 1, pp. 154-156 2007. 
[144] G. K. Feld, R. P. Fleck, O. Fujimura, D. L. Prothro, T. D. Bahnson, and M. Ibarra, "Control of rapid ventricular response by radiofrequency catheter modification of the atrioventricular node in patients with medically refractory atrial fibrillation," Circulation, vol. 90, no. 5, pp. 2299-2307 1994

[145] B. D. Williamson, K. C. Man, E. Daoud, M. Niebauer, S. A. Strickberger, and F. Morady, "Radiofrequency catheter modification of atrioventricular conduction to control the ventricular rate during atrial fibrillation," The New England Journal of Medicine, vol. 331, no. 14, pp. 910-917 1994.

[146] T. N. Mazgalev and P. J. Tchou, "Surface potentials from the region of the atrioventricular node and their relation to dual pathway electrophysiology," Circulation, vol. 101, no. 17, pp. 2110-2117 2000.

[147] M. L. Young, C. T. Kuo, V. Kohli, and G. S. Wolff, "Similar timedependent recovery property of fast and slow atrioventricular nodal pathways," American Journal of Cardiology, vol. 79, no. 4, pp. 424-430 1997.

[148] T. C. Coleman and Y. Li, "On the convergence of interior-reflective newton methods for nonlinear minimization subject to bounds," Mathematical Programming, vol. 67, no. 2, pp. 189-224 1994.

[149] T. F. Coleman and Y. Li, "An interior trust region approach for nonlinear minimization subject to bounds," SIAM Journal on Optimization, vol. 6, no. 2, pp. 418-445 1996.

[150] P. Denes, D. Wu, R. C. Dhingra, R. Chuquimi, and K. M. Rosen, "Demonstration of dual A-V nodal pathways in patients with paroxysmal supraventricular tachycardia," Circulation, vol. 48, no. 3, pp. 549-555 1973.

[151] M. Haissaguerre, F. Gaita, B. Fischer, D. Commenges, P. Montserrat, C. d'Ivernois, P. Lemetayer, and J. F. Warin, "Elimination of atrioventricular nodal reentrant tachycardia using discrete slow potentials to guide application of radiofrequency energy," Circulation, vol. 85, no. 6, pp. 2162-2175 1992 .

[152] W. M. Jackman, K. J. Beckman, J. H. Mcclelland, X. Z. Wang, K. J. Friday, C. A. Roman, K. P. Moulton, N. Twidale, H. A. Hazlitt, M. I. Prior, J. Oren, E. D. Overholt, and R. Lazzara, "Treatment of supraventricular tachycardia due to atrioventricular nodal reentry by radiofrequency catheter ablation of slow-pathway conduction," New England Journal of Medicine, vol. 327, no. 5, pp. 313-318 1992. 
[153] P. Denes, L. Levy, A. Pick, and K. M. Rosen, "Incidence of typical and atypical AV Wenckebach periodicity," American Heart Journal, vol. 89, no. 1, pp. 26-31 1975.

[154] W. Zeng, T. N. Mazgalev, A. Munk, A. Shrier, and J. Jalife, Dual Atrioventricular Pathways Revisited: On the cellular mechanism of discontinuous $A V$ nodal recovery and the gap phenomenon, ser. Cardiac Electrophysiology: from Cell to Bedside. Philadelphia: WB Saunders Company, 1995, pp. 314-325.

[155] W. J. Hucker, M. McCain, J. Laughner, P. Iaizzo, and I. R. Efimov, "Connexin 43 expression delineates two discrete pathways in the human atrioventricular junction." Anatomical Record(Hoboken), vol. 291, pp. 204-215 2008.

[156] C. T. Tai, S. A. Chen, C. E. Chiang, S. H. Lee, C. W. Chiou, K. C. Ueng, Z. C. Wen, Y. J. Chen, and M. S. Chang, "Multiple anterograde atrioventricular node pathways in patients with atrioventricular node reentrant tachycardia," Journal of the American College of Cardiology, vol. 28, no. 3, pp. 725-731 1996.

[157] I. R. Efimov and T. N. Mazgalev, "High-resolution, three-dimensional fluorescent imaging reveals multilayer conduction pattern in the atrioventricular node," Circulation, vol. 98, no. 1, pp. 54-57 1998.

[158] A. M. Climent, F. Atienza, J. Millet, and M. S. Guillem, "Generation of realistic atrial to atrial interval series during atrial fibrillation," under review 2011.

[159] P. Bonizzi, M. S. Guillem, A. M. Climent, J. Millet, V. Zarzoso, F. Castells, and O. Meste, "Noninvasive assessment of the complexity and stationarity of the atrial wavefront patterns during atrial fibrillation," IEEE Transactions on Biomedical Engineering, vol. 57, no. 9, pp. 214721572010.

[160] M. S. Guillem, A. M. Climent, F. Castells, D. Husser, J. Millet, A. Arya, C. Piorkowski, and A. Bollmann, "Noninvasive mapping of human atrial fibrillation," Journal of Cardiovascular Electrophysiology, vol. 20, no. 5, pp. 507-513 2009.

[161] M. S. Guillem, A. M. Climent, A. Bollmann, D. Husser, J. Millet, and F. Castells, "Limitations of Dower's inverse transform for the study of atrial loops during atrial fibrillation," Pacing and Clinical Electrophysiology, vol. 32, no. 8, pp. 972-980 2009.

[162] M. S. Guillem, A. V. Sahakian, and S. Swiryn, "Derivation of orthogonal leads from the 12-lead electrocardiogram. Performance of an atrial-based 
transform for the derivation of P loops," Journal of Electrocardiology, vol. 41, no. 1, pp. 19-25 2008.

[163] M. S. Guillem, A. Bollmann, A. M. Climent, D. Husser, J. Millet, and F. Castells, "How many leads are necessary for a reliable reconstruction of surface potentials during atrial fibrillation?" IEEE Transactions on Information Technology in Biomedicine, vol. 13, no. 3, pp. 330-340 2009.

[164] V. Donis, M. S. Guillem, A. M. Climent, F. Castells, F. J. Chorro, and J. Millet, "Improving the diagnosis of bundle branch block by analysis of body surface potential maps," Journal of Electrocardiology, vol. 42, no. 6, pp. 651-659 2009.

[165] A. M. Climent, M. S. Guillem, D. Husser, F. J. Castells, J. Millet, and A. Bollmann, "Circadian rhythm of ventricular response during atrial fibrillation is not determined by dual atrioventricular nodal pathway conduction," International Society for Computerized Electrocardiology Meeting Proceedings 20072007.

[166] A. M. Climent, M. S. Guillem, Y. Zhang, J. Millet, and T. N. Mazgalev, "Functional model of dual AV nodal pathway physiology," IEEE Engineering in Medicine and Biology Society.Conference, vol. 1, pp. 2646-2649 2010. 